\title{
Fall prevention in psychogeriatric nursing home residents
}

Citation for published version (APA):

Neyens, J. C. L. (2007). Fall prevention in psychogeriatric nursing home residents. [Doctoral Thesis, Maastricht University]. D\&L Graphics. https://doi.org/10.26481/dis.20070614jn

Document status and date:

Published: 01/01/2007

DOI:

10.26481/dis.20070614jn

Document Version:

Publisher's PDF, also known as Version of record

\section{Please check the document version of this publication:}

- A submitted manuscript is the version of the article upon submission and before peer-review. There can be important differences between the submitted version and the official published version of record.

People interested in the research are advised to contact the author for the final version of the publication, or visit the DOI to the publisher's website.

- The final author version and the galley proof are versions of the publication after peer review.

- The final published version features the final layout of the paper including the volume, issue and page numbers.

Link to publication

\footnotetext{
General rights rights.

- You may freely distribute the URL identifying the publication in the public portal. please follow below link for the End User Agreement:

www.umlib.nl/taverne-license

Take down policy

If you believe that this document breaches copyright please contact us at:

repository@maastrichtuniversity.nl

providing details and we will investigate your claim.
}

Copyright and moral rights for the publications made accessible in the public portal are retained by the authors and/or other copyright owners and it is a condition of accessing publications that users recognise and abide by the legal requirements associated with these

- Users may download and print one copy of any publication from the public portal for the purpose of private study or research.

- You may not further distribute the material or use it for any profit-making activity or commercial gain

If the publication is distributed under the terms of Article $25 \mathrm{fa}$ of the Dutch Copyright Act, indicated by the "Taverne" license above, 


\section{Fall prevention in psychogeriatric nursing home residents}

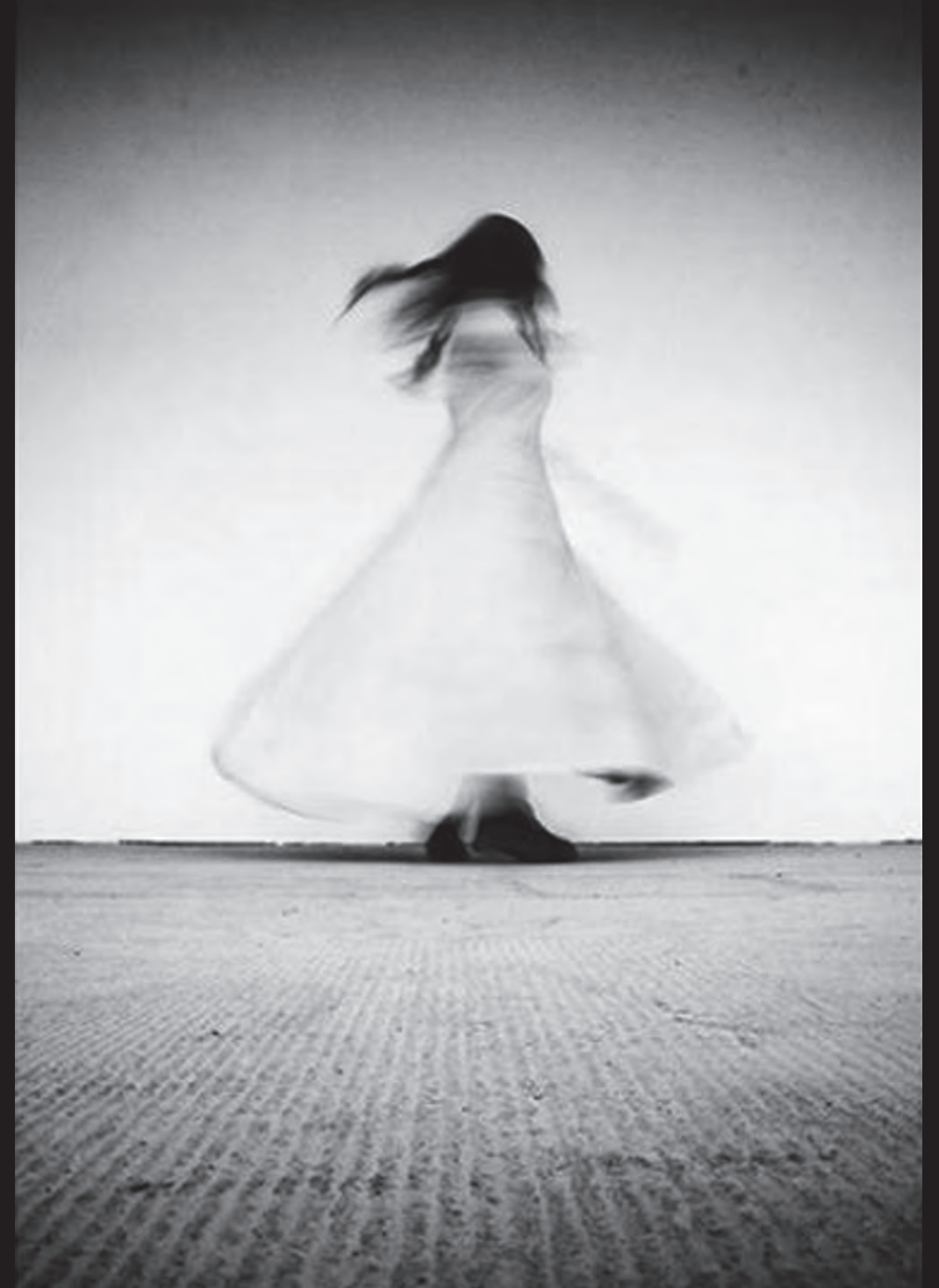

Jacques Neyens 


\section{Fall prevention in psychogeriatric nursing home residents}

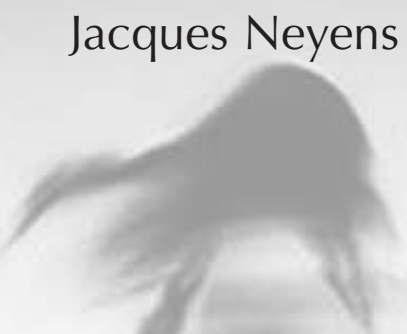

1 Dat in Nederlandse verpleeghuizen jaarlijks bijna twee valincidenten per bed plaatsvinden, is zorgelijk. (dit proefschrift)

2 Een specifieke valrisico-evaluatie voor psychogeriatrische verpleeghuispatiënten leidt tot op maat gesneden valpreventieve maatregelen. (dit proefschrift)

3 De implementatie van een multifactoriële interventie ter preventie van vallen bij psychogeriatrische patiënten in verpleeghuizen, leidt tot een significante vermindering van het aantal valincidenten. (dit proefschrift)

4 Bij de implementatie van een valpreventieve interventie voor verpleeghuispatiënten dient ervoor gezorgd te worden dat deze naadloos aansluit bij het gangbare zorgproces in het verpleeghuis. (dit proefschrift)

5 Nader onderzoek doen naar de verdere optimalisering van valpreventieve interventies in verpleeghuizen is noodzakelijk. (dit proefschrift)

6 Een mens is vanwege zijn lengte en zijn smalle steunbasis eigenlijk voorbestemd om te vallen. (W. Pelemans, in Geriatrie-Dagelijkse Praktijk, 1998.)

7 Op momenten dat de bezettingsgraad van het verzorgend personeel in het verpleeghuis laag is, neemt de valfrequentie toe. (L.Z. Rubenstein, in Clinics in Geriatr Med, 1996.)

8 In het streven naar een optimale patiëntveiligheid behoren risico-identificatie en risicovermindering tot de verantwoordelijkheid van alle gezondheidswerkers.

9 Werken aan de kwaliteit van de verpleeghuiszorg gebeurt met vallen en opstaan!

10 Als het is om vallen te voorkomen, dan zou het vastzetten van verpleeghuisbewoners bij wet moeten worden verboden. (J.P.H. Hamers, 2007.)

11 Oud worden valt niet mee. 


\section{Fall prevention in psychogeriatric nursing home residents}

Jacques Neyens 
The studies presented in this thesis were performed at the Institute for Rehabilitation Research (iRv), the Netherlands. The iRv participates in the Netherlands School of Primary Care Research (CaRe), which was acknowledged in 1995 by the Royal Netherlands Academy of Arts and Sciences (KNAW).

Financial support to perform this research project was provided by Stichting De Riethorst Stromenland, Geertruidenberg, and by the Institute for Rehabilitation Research (iRv), Hoensbroek.

The publication of this thesis was kindly financed by Stichting WSG, Geertruidenberg.

Cover: "Guardian Angel"

(C) 2007, Sanne Neyens (coverdesign, fashion and model), Brecht, Belgium.

ISBN: 978-90-8590-021-4

(C) 2007, J.C.L. Neyens, Brecht, Belgium.

All rights reserved. No part of this thesis may be reproduced or transmitted in any form or by any means, electronic or mechanical, including photocopying, recording or any information storage or retrieval system, without permission in writing from the author, or, when appropriate, from the publishers of the publications.

Lay-out and Design:

D\&L Graphics

www.dlgraphics.nl

info@dlgraphics.nl

Published by:

D\&L Graphics

Printed by:

Schrijen Lippertz-Huntjens 


\title{
Fall prevention in psychogeriatric nursing home residents
}

\author{
PROEFSCHRIFT \\ ter verkrijging van de graad van doctor \\ aan de Universiteit Maastricht, \\ op gezag van de Rector Magnificus, \\ Prof. mr. G.P.M.F Mols, \\ volgens het besluit van het College van Decanen, \\ in het openbaar te verdedigen \\ op donderdag 14 juni 2007 om 16.00 uur
}

door

Jacques Christiaan Luc Neyens

Geboren te Bree op 29 augustus 1956 


\section{Promotores}

Prof. dr. W.J.A. van den Heuvel

Prof. dr. J.M.G.A. Schols

\section{Co-promotores}

Dr. L.P. de Witte

Dr. J.C.M. van Haastregt

\section{Beoordelingscommissie}

Prof. dr. C. Spreeuwenberg (voorzitter)

Prof. dr. R.A. de Bie

Prof. dr. H.J.M. Cools (LUMC)

Prof. dr. G.I.J.M. Kempen

Prof. dr. K. Milisen (KU Leuven, België)

The main study of this thesis, the randomised controlled trial on fall prevention in psychogeriatric nursing home residents, was funded by the Netherlands Organisation for Health Research and Development (ZonMw). The additional process evaluation was funded by the Foundation to promote Nursing Home Research (SWBV). 
Voor Mady, Maarten en Sanne 



\section{Contents}

$\begin{array}{lll}\text { Chapter } 1 \text { General introduction } & 9\end{array}$

Chapter 2 Falls in nursing homes: almost two per bed per year,

$1.3 \%$ resulting in fracture

Chapter 3 Effects and feasibility of interventions for preventing falls in elderly people in long-term care facilities: systematic review

Chapter 4 The development of a multidisciplinary fall risk evaluation tool for demented nursing home patients in the Netherlands

Chapter 5 A multifactorial intervention for the prevention of falls in psychogeriatric nursing home patients,

a randomised controlled trial

Chapter 6 Feasibility of a multifactorial fall prevention programme for psychogeriatric nursing home patients

Chapter $7 \quad \mathrm{CBO}$ guidelines to prevent falls in the elderly:

how can they be used in the institutionalised elderly?

Chapter 8 General discussion

Summary

Samenvatting

Dankwoord

Curriculum Vitae 



\section{Chapter 1}

\section{General introduction}


Chapter 1 


\section{General introduction}

This thesis reports on extensive research into preventing falls and fall-related injuries among institutionalised elderly persons with psychogeriatric disorders. It also describes the development of a multifactorial and multidisciplinary fall prevention programme for demented nursing home patients in The Netherlands as well as the evaluation of the effectiveness and feasibility of this intervention. With our research, we also aimed to improve the implementation of fall prevention activities in daily nursing home care, by raising professional awareness about falls by nursing home patients generally and by demented nursing home patients in particular.

Long-term care residents have a higher fall incidence than communitydwelling elderly. ${ }^{1-3}$ Therefore, falls are a major problem for both residents and the institutions, as they lead to increased morbidity, secondary mortality, and a higher health care consumption. In order to decrease the number of falls and fall-related injuries in nursing home residents, it is important that long-term care facilities establish an adequate and effective fall prevention policy.

In this introductory chapter we start with a description of the incidence and the consequences of falls among elderly persons in general and in the Netherlands in specific. Secondly, we present a short overview of risk factors for falling and the possibilities of fall prevention. Thirdly, we present the main characteristics of nursing homes in the Netherlands and finally we present the objectives, research questions and outline of the thesis.

\section{FALLS IN THE ELDERLY}

\section{General}

A fall is defined as an unintentional change in position that results in a person coming to rest on the ground or other lower level. ${ }^{4}$ Falls are a major problem for a growing number of elderly people. The incidence of falls increases strongly with age. ${ }^{5-8}$ Thirty percent of community-dwelling persons aged 65 years or more have at least one fall a year, and about $15 \%$ have two or more falls a year. $6,7,9-11$ The fall rate is even higher for frail elderly residing in long-term care institutions: $30-70 \%$ fall at least once a year and $15-40 \%$ fall two or more times a year. ${ }^{1-3,7,8,12-14}$

Falls may have serious consequences, such as fractures or other major injuries, which may lead to hospital admission or even death. Fall-related injuries are the third leading cause of years lived with disability according to the WHO. 5, 7,8,15 


\section{Chapter 1}

The majority of fall-related injuries are minor, e.g. bruises that do not require medical treatment. ${ }^{5,7,8,10,11,16}$ Five to ten percent of falls result in major injuries requiring medical treatment, e.g. hip fractures (1-2\%), other fractures $(3-5 \%)$, soft tissue injuries and head trauma (5\%). $5,7,8,11,13,16,17$ Even if a fall does not result in physical injury, there may be a severe psychological impact such as fear of falling, which may lead to decreased mobility, restrictions in activities or social functioning, and increased dependency. ${ }^{18,19}$

The costs associated with falls and fall-related injuries are substantial and directly related to the fall frequency and severity of injury. ${ }^{20-22}$ Furthermore, falls and related injuries increase the nursing care load. Although fall prevention may be expensive and time- consuming, it is of high clinical relevance due to the high incidence of falls in the elderly, and the morbidity and mortality resulting from falls. $5,15,23,24$

In summary, fall prevention in the elderly is of great importance and high clinical relevance.

\section{Falls in the Netherlands}

In 2004, about 2.2 million people in the Netherlands were aged 65 years or more $(13.7 \%$ of the total population). Of these, about 110,000 lived in old people's homes and 57,000 in nursing homes. ${ }^{25}$

Thirty percent of elderly people living at home and $50 \%$ of elderly residents in long-term care facilities fall at least once per year. ${ }^{26}$ This means that, in 2004, more than 650,000 Dutch elderly fell at least once. In the Netherlands, the total number of falls by people aged 65 years or more is almost one million per year. Falls are the main cause of accidental death in this group. ${ }^{26}$ It is estimated that the 170,000 residents in long-term care facilities account for about 223,000 falls per year (112,000 in old people's homes and 110,000 in nursing homes, that is 1.31 falls per resident per year). ${ }^{27}$ By comparison, two million elderly living at home account for 677,000 falls per year, that is 0.34 falls per elderly living at home per year. With the total number of persons aged 65 years or over, increasing to 3.8 million in 2030, it is clear that the absolute number of falls in this population will rise substantially in the next decades. ${ }^{26}$

Every year, 36,000 people aged 55 years or more require emergency hospital treatment following a fall at home. ${ }^{26}$ About $70 \%$ suffer minor injuries, while $6 \%$ have major injuries, including fractures. Almost $25 \%$ need medical or paramedical treatment. The main consequences of falls are decreased mobility $(30 \%)$, restrictions in social functioning $(17 \%)$, and restrictions in activities $(15 \%) .{ }^{26}$ These consequences may eventually lead to loss of autonomy and even death. With regard to residents in long-term care facilities, 4,500 persons 
per year need treatment in hospital emergency units after a fall. ${ }^{26}$ The hip fracture rate in this population is about 1500 per year (34\%). The hip fracture rate in community-dwelling elderly is $8 \%$. Overall, there were about 15,000 fall-related hip fractures in the Netherlands among persons aged 55 years or more in 2001. ${ }^{28}$ This number is expected to rise to 20,000 in 2010. One-quarter of persons with a hip fracture are permanently disabled; another $25 \%$ die within a year.

The costs associated with falls and fall-related injuries are substantial. In 1993, the direct medical cost of fractured hips, vertebrae and forearms was calculated at 190 million euros. ${ }^{21}$ In 1998-2001, the direct medical cost of falls was, on average, 3,400 euros per fall. ${ }^{29}$ Other costs such as physiotherapy and home care were not taken into account. By now, these costs will inevitable have risen further.

These data underscore the importance of fall prevention for the elderly in general, and in particular for nursing home residents.

\section{RISK FACTORS FOR FALLS}

Researchers have identified numerous risk factors for falls. These can be subdivided into intrinsic (related to the individual) and extrinsic factors (related to the environment). Fall risk increases with the number of risk factors present. ${ }^{8,27}$ The literature does not provide univocal information about the relative contribution of fall risk factors to overall risk. This can be explained by differences in population, methodology and statistical techniques. ${ }^{10}$

Several studies have shown the importance of intrinsic risk factors. ${ }^{8,11,14,16}$ Intrinsic factors which are reported to be significantly associated with increased fall risk are advanced age $\mathrm{e}^{7,14,30-33}$, high level of dependence $7,8,10,27,31,33-35$, previous falls $8,10,13,27,30-33,35-37$, impaired balance ${ }^{5,7,8,10,11,13,14,27,30,31,33,36,39-42}$, muscle weakness $5,7,8,10,11,13,14,27,36,39-41$, gait disorders 5,7,8,10, 11,13,14,27,36,39,40, use of psychotropic drugs $8,10,13,14,27,30,32,33,35-37,42$, taking more than four medications ${ }^{43,44}$, medical conditions (e.g. hypotension, cardiovascular disease, stroke, urinary incontinence, visual impairment, and dizziness) $)^{5,7,8,10,13,14,27,36,39,40}$, psychosocial factors like depression $8,10,13,27,30,36,39,40,45$, and low levels of social support. ${ }^{10,11,27,36}$ There is also an association between falls and cognitive functioning. ${ }^{13,14,27,46,47}$ Dementia is one of the most important causes of disability in the elderly. Patients with dementia suffer gradually deteriorating mobility and have a diminishing ability to recognise, judge and avoid hazards. This makes dementia a major risk factor for falling. ${ }^{1,2,48,49}$ The prevalence of dementia increases exponentially in the most advanced ages from $2.1 \%$ in the 


\section{Chapter 1}

70-74 age group to $32 \%$ in the $85-90$ age group in the Netherlands. ${ }^{50}$ Van Dijk et al. studied falls in psychogeriatric nursing home patients and reported a mean of 4.1 falls per person-year. The fall rate was at its highest the first week after admission. ${ }^{2}$

Extrinsic risk factors that may contribute to falls are: thresholds, stairs, poor lighting, slippery floors, inappropriate footwear and so on. At present, however, the relationship between these factors and falls has not been established convincingly. ${ }^{51,52}$ Extrinsic factors may play a supplementary role, heightening the effect of intrinsic factors. ${ }^{5,7,8,13}$ Environmental hazards may also interact critically with individual condition and behaviour. ${ }^{53}$

Only a small number of studies have assessed risk factors for falls in patients with dementia. ${ }^{2,54-56}$ Brody et al. studied institutionalised women with Alzheimer's disease and found that the main predictor of falls is a relatively high initial physical vigour, followed by a significant decline in vigour in the subsequent year. ${ }^{54}$ Van Dijk et al. found that fall risk in institutionalised dementia patients increased to a certain score on a care dependency scale and then declined, indicating that severely demented patients may have a relatively lower fall risk because they are often chairbound or bedridden. ${ }^{2}$ Due to agitation, restlessness and other behavioural problems, restraints are often used in demented patients, mostly with regard to fall prevention. ${ }^{57}$ However, restraint reduction reduces serious injuries among nursing home residents. ${ }^{58,59}$ Buchner et al. found no relationship between falls and severity of dementia but did find an association between wandering and fractures, including hip fractures. ${ }^{55}$ Steinberg et al. reported that $23 \%$ of demented residents fell in the first week after admission. ${ }^{56}$ Predictors of falls were: poor general medical health, falls in the two weeks prior to admission and greater impairment in activities of daily living. An interaction emerged between wandering and health status, with wanderers in excellent or good health being at particular risk of falling at least once. Steinberg et al. concluded that general medical health is a powerful independent predictor of fall risk, which may indicate that poor physical condition is the most important factor of increasing fall risk in demented elderly persons, at least in patients who do not wander. ${ }^{56}$

Fall prevention in institutionalised elderly should be targeted at these risk factors for falling.

\section{FALL PREVENTION}

Once, falls were defined as unpredictable incidents, an unavoidable side effect of illness, disability or advanced age. Falls were accepted as a normal consequence of being ill, and fall-related injuries were accepted as "bad luck". ${ }^{60}$ Today, the general 
conviction is that falls can be predicted with reasonable certainty and are therefore preventable. ${ }^{60}$ In the last decades, a lot of scientific literature has become available about preventing falls in the elderly. ${ }^{23,36,61-63} \mathrm{~A}$ variety of fall prevention interventions, targeting one or more fall risk factors, have been developed, investigated and evaluated. ${ }^{61}$ Although some focus on a single risk factor, most interventions are directed at multiple risk factors: exercise programmes to improve strength or balance, educational programmes, optimisation of medication intake, environmental modification in homes or institutions, and nutritional supplementation. Research has indicated that these prevention activities have positive effects on the number of fall incidents. ${ }^{61,64}$ Most studies, however, concerned community-dwelling people. Despite the magnitude of the problem of fall incidents in institutionalised elderly, there is considerably less evidence about fall prevention in this group. ${ }^{61.65}$

There are several guidelines on fall prevention in the elderly, based on the findings of research. ${ }^{26,36,61,66,67}$ Most, however, concern community-dwelling elderly. When the present research was launched, the Netherlands had no national fall prevention guidelines. Now, there are two. ${ }^{26,68}$ The guideline "Fall prevention in the elderly" focuses on fall prevention in the community, longterm care settings and hospitals. ${ }^{26}$ The guideline follows an evidence-based approach with general recommendations for each setting, including nursing homes. The guideline "Fall prevention by occupational therapists" focuses on what occupational therapists can do with regard to fall prevention in independent elderly, living at home or in long-term care institutions. ${ }^{68}$

\section{Nursing homes in the Netherlands}

In the Netherlands, a relatively high percentage of elderly older than 65 years $(7 \%)$ live in homes for the elderly or nursing homes. ${ }^{69}$

A Dutch nursing home is "an institution which provides temporary or permanent multidisciplinary treatment, guidance, support and nursing care for elderly patients with long-term, complex health problems, expressed primarily in functional disorders and handicaps". ${ }^{69-73}$ There are about 330 nursing homes in the Netherlands, with about 57,000 patients. ${ }^{74}$ Most have somatic wards for patients with physical disabilities and psychogeriatric wards for patients with dementia syndromes. The impairments and disabilities seen in nursing home residents often affect multiple domains of functioning: physical, psychological and social. The geriatric profile of a "typical" nursing home patient is characterised by considerable co-morbidity, multiple disabilities, frequent disorientation, confusion or mood problems, and polypharmacy. 


\section{Chapter 1}

In 1990, "nursing home medicine" was recognised as a medical specialty for which specialist training and registration are required. ${ }^{75,76}$ Nursing home physicians work in a multidisciplinary nursing home team consisting of nurses, physiotherapists, occupational therapists, psychologists, speech therapists, dieticians, social workers, pastoral workers and recreational therapists. ${ }^{69,73,77}$ These care teams formulate tailor-made care plans for each individual patient. Plans are evaluated and adjusted on a regular basis, together with the patient and his or her relatives. This multidisciplinary, periodically evaluated systematic approach characterises the delivery of care in Dutch nursing homes. ${ }^{78}$

Most somatic nursing home patients are referred by the hospital (65\%) or their family physicians (26\%). Psychogeriatric patients come from their own homes $(53 \%)$, residential homes $(23 \%)$ or hospitals $(20 \%) .{ }^{70,79-81}$ Many of the somatic patients in nursing homes nowadays are there temporarily for rehabilitation. Mainly, the long-term rehabilitation provided ties in better with the clinical profile and activity level of the older patient. The aim of rehabilitation is to enable patients to go back to living at home, or in a home for the elderly. The remaining somatic patients and overriding majority of psychogeriatric patients in nursing homes stay there until death. ${ }^{70.79-81}$

Nursing home care includes observation and assessment, nursing care, reactivation/rehabilitation, long-term treatment, support, palliative care and crisis intervention. ${ }^{82}$ Falls in the elderly are a strong predictor for admission to a nursing home. ${ }^{24}$ About $50 \%$ of fall-related injuries that occur at home and require hospital admission result in discharge to a nursing home. ${ }^{17}$ Eight percent of geriatric women with fractures of the hip, spine, or distal forearm need nursing home care. ${ }^{16}$ Falls are also common in nursing homes themselves, with a fall rate of approximately 2 per person per year in the Netherlands. ${ }^{74,83}$

As mentioned before, falls are associated with cognitive decline. ${ }^{1,2,13,14,27,46,47}$ About $55 \%$ of the 57,000 patients in Dutch nursing homes have dementia. These patients account for $75 \%$ of the fall incidents. ${ }^{69}$ It is safe to say that patients in nursing homes, in particular those with dementia, have a high risk of falling. ${ }^{84}$ This underscores the importance of adequate fall prevention activities for this group of patients.

Until recently, scientific research in Dutch nursing homes was scarce. Now, however, a growing number of scientific studies has been launched in these settings, either by nursing homes themselves and/or in cooperation with universities and/or research institutes. ${ }^{73,85}$ 


\section{ObJeCtiVES AND RESEARCH QUESTIONS}

Falls in psychogeriatric nursing home patients are a major problem. This thesis examines how falls in this population might be reduced. The aims of our research were:

- to increase professional awareness about the problem of falling in nursing home patients generally, and in demented patients in particular;

- to give an overview of the effectiveness and feasibility of fall prevention interventions in long-term care facilities;

- to evaluate a new multifactorial fall prevention intervention for psychogeriatric nursing home patients on its effectiveness and feasibility;

- to contribute to the development of guidelines on fall prevention in longterm care

The main research questions to be answered in this thesis are:

- what is the effectiveness of the new multifactorial fall prevention intervention among psychogeriatric nursing home patients in the Netherlands?

- to what extend is this multifactorial fall prevention programme feasible in daily nursing home practice?

\section{OUtLINE OF THE THESIS}

This thesis reports on the development, effectiveness and feasibility of a multifactorial fall prevention intervention in psychogeriatric nursing home patients. Chapter 2 reports on the incidence of falls in Dutch nursing homes based on a national survey. Chapter 3 presents a systematic review with regard to the effects and feasibility of interventions for preventing falls in elderly people in long-term care facilities. This review covers randomised controlled trials performed in the last decades. Chapter 4 describes the development of the multidisciplinary fall risk evaluation tool that is an essential component of our multidisciplinary fall prevention intervention for demented nursing home patients in the Netherlands. Chapter 5 presents the results of a randomised controlled trial assessing the effectiveness of a multifactorial intervention for the prevention of falls and fall-related injuries in psychogeriatric nursing home patients. Chapter 6 reports on the results of a process evaluation in which we assessed the feasibility of our fall prevention programme for daily nursing home practice. In chapter 7, we summarise the essentials for nursing homes with regard to the first national guideline "Fall prevention in the elderly". We helped 


\section{Chapter 1}

draw up this guideline, which incorporates some of our early study findings. Finally, chapter 8 contains a general discussion of the results of the studies presented in this thesis, as well as recommendations for daily health care practice and future research. 


\section{References}

1. Vermeulen HBM (1994). Vallen en valpreventie in het verpleeghuis: een interventiestudie naar het effect van een bed-alarm-systeem. Nijmegen, academisch proefschrift Katholieke Universiteit Nijmegen.

2. Dijk van PTM, Meulenburg OGRM, Sande HJ van de, Habbema JDF. Falls in dementia patients. Gerontologist 1993; 33(2): 200-204.

3. Berry G, Fisher R, Lang S. Detrimental incidents including falls in an elderly institutionalized population. Journal of the American Gerontolopical Association 1981; 29: 322-24.

4. Kellogg International Work Group on the prevention of falls in the elderly. The prevention of falls in later life. Dan Med Bull 1987; 34(suppl)4:1-24.

5. Campbell AJ, Borrie MJ, Spears GF, Jackson SL, Brown JS, Fitzgerald JL. Circumstances and consequences of falls experienced by a community population 70 years and over during a prospective study. Age Ageing 1990; 19: 136-41.

6. Blake AJ, Morgan K, Bendall MJ, Dallosso H, Ebrahim SBJ, Arie THD, et al. Falls by elderly people at home: prevalence and associated factors. Age Ageing 1988; 17: 365-72.

7. O'Loughlin JL, RobitailleY, Boivin J, Suissa S. Incidence of risk factors for falls and injurious falls among the community-dwelling elderly. Am J Epidemiol 1993; 137: 342-54.

8. Tinetti ME, Speechley M, Ginter SF. Risk factors for falls among elderly persons living in the community. N Engl J Med 1988; 319: 1701-7.

9. Luukinen H, Koski K, Hiltunen L, Kivela SL. Incidence rates of falls in an aged population in northern Finland. J Clin Epidemiol 1994; 8: 843-50.

10. Stalenhoef PA, Crebolder HFJM, Knottnerus JA, van der Horst FGEM. Incidence, risk factors and consequences of falls among elderly subjects living in the community: a criteria-based analysis. Eur J Publ Health 1997; 7: 328-34.

11. Tromp AM, Smit JH, Deeg DJH, Bouter LM, Lips P. Predictors for falls and fractures in the longitudinal Aging Study Amsterdam. J Bone Min Res 1998; 13: 1932-9.

12. Dijcks BPJ, Neyens JCL, Schols JMGA, van Haastregt JCM, Crebolder HFJM, de Witte LP. Valincidenten in verpleeghuizen: gemiddeld bijna 2 per bed per jaar met bij 1,3\% een fractuur als gevolg. Ned Tijdschr Geneeskd 2005; 149: 1043-7.

13. Nevitt MC, Cummings SR, Kidd S, Black D. Risk factors for recurrent nonsyncopal falls. A prospective study. JAMA 1989; 261: 2663-8.

14. Campbell AJ, Borrie MJ, Spears GF. Risk factors for falls in a community based prospective study of people 70 years and older. J Geront Med Sci 1989; 44: 112-7.

15. Murray CJL, Lopez AD. Global and regional descriptive epidemiology of disability: incidence, prevalence, health expectancies and years lived with disability. In: Murray CJL, Lopez AD, eds. The global burden of disease. Boston Harvard University Press; 1996: 201-46.

16. Nevitt MC, Cummings SR, Hudes ES. Risk factors for injurious falls: a prospective study. J Gerontol 1991; 46: M164-70. 


\section{Chapter 1}

17. Sattin RW, Lambert Huber DA, DeVito CA, Rodriguez JG, Ros A, Bacchelli S, Stevens JA, Waxweiler RJ. The incidence of fall injury events among the elderly in a defined population. Am J Epidemiol 1990; 131: 1028-37.

18. Tinetti ME, Mendes de Leon CF, Doucette JT, Baker DI. Fear of falling and fall-related efficacy in relationship to functioning among community living elders. / Gerontol 1994; 49: M140-47.

19. Vellas BJ, Wayne SJ, Romero LJ, Baumgartner RN, Garry PJ. Fear of falling and restriction of mobility in elderly fallers. Age Ageing 1997; 26: 189-93.

20. Alexander BH, Rivara FP, Wolf ME. The cost and frequency of hospitalisation for fallrelated injuries in older adults. Am / Public Health 1992; 82: 1020-3.

21. de Laet $\mathrm{CE}$, van Hout BA, Burger H, Weel AE, Hofman A, Pols HA. Incremental cost of medical care after hip fracture and first vertebral fracture: the Rotterdam Study. Osteoporos 1999; 10: 66-72.

22. Rizzo JA, Baker DI, McAvay G, Tinetti ME. The cost-effectiveness of a multifactorial targeted prevention program for falls among community elderly persons. Med Care 1996; 34: 954-69.

23. Boers I, Willibald G, Stalenhoef PA, Bloem BR (2001). Falls in the elderly. Wien Klin Wochenschr 2001; 113/11-12: 398-407.

24. Tinetti ME, Williams CS. Falls, injuries due to falls, and the risk of admission to a nursing home. N Engl / Med 1997; 337: 1279-84.

25. SCP-rapportage ouderen 2004. Zorg en wonen voor kwetsbare ouderen. (Red.MMY de Klerk). Den Haag: Sociaal Cultureel Planbureau, mei 2004.

26. СВO, Kwaliteitsinstituut voor de Gezondheidszorg. Richtlijn Preventie van valincidenten bij ouderen. Alphen aan de Rijn: Van Zuiden Communications BV, 2004.

27. Graafmans WC, Ooms ME, Hofstee HMA, Bezemer PD, Bouter ML, Lips P. Falls in the elderly: a prospective study of risk factors and risk profiles. Am J Epidemiol 1996; 143: 1129-36.

28. $\mathrm{CBO}$, Kwaliteitsinstituut voor de Gezondheidszorg. Richtlijn Osteoporose. Alphen aan de Rijn: Van Zuiden Communications BV, 2002.

29. http://statline.cbs.nl

30. Bueno-Cavanillas A, Padilla-Ruiz F, Jiménez-Moleón JJ, Peinado-Alonso CA, GálvezVargas R. Risk factors in falls among the elderly according to extrinsic and intrinsic precipitating causes. Eur J Epidemiol 2000; 16: 849-859.

31. Kiely DK, Kiel DP, Burrows AB, Lipsitz LA. Identifying nursing home residents at risk for falling. JAGS 1998; 46: 551-555.

32. Myers AH, Baker SP, Van Natta ML, Abbey H, Robinson EG. Risk factors associated with falls and injuries among elderly institutionalized persons. Am J Epidemiol 1991; 133: 1179-1190.

33. Thapa PB, Gideon P, Brockman KG, Fought RL, Ray WA. Clinical and biomechanical measures of balance as fall predictors in ambulatory nursing home residents. J Gerontol Med Sci 1996; 51A: M239-M246. 
34. Graafmans WC, Lips P, Wijlhhuizen GJ, Pluijm SM, Bouter LM. Daily physical activity and the use of a walking aid in relation to falls in elderly people in a residential care setting. Z Gerontol Geriatr 2003; 36: 23-28.

35. Tromp AM, Pluijm SMF, Smit HJ, Deeg DJH, Bouter LM, Lips P. Fall-risk screening test: a prospective study on predictors for falls in community-dwelling elderly. I Clin Epidemiol 2001; 54: 837-44.

36. Krueger PD, Brazil K, Lohfeld LH. Risk factors for falls and injuries in a long-term care facility in Ontario. Can J Public Health 2001; 92: 117-120.

37. Nurmi I Sihvonen M, Kataja M, Lüthje P. Falls among institutionalized Elderly a prospective study in four institutions in finland. Scand / Caring Sci 1996; 10: 212-220.

38. American Geriatrics Society, British Geriatrics Society and American Academy of Orthopaedic Surgeons Panel on Fall Prevention. Guideline for the Prevention of Falls in Older Persons. JAGS 2001; 49: 664-672.

39. Clark RD, Lord SR, Webster IW. Clinical parameters associated with falls in an elderly population. Gerontology 1993; 39: 117-23.

40. Tinetti ME, Inouye SK, Gill TM, Doucette JT. Shared risk factors for falls, incontinence, and functional dependence: unifying the approach to geriatric syndromes. JAMA 1995; 273: 1348-53.

41. Luukinen H, Koski K, Laippala P, S-L Kivela. Risk factors for recurrent falls in the elderly in long-term institutional care. Pub Health 1995; 109: 57-65.

42. Yip YB, Cumming RG. The association between medications and falls in Australian nursing-home residents. Med J Aust 1994; 160: 14-18.

43. Leipzig RM, Cumming RG, Tinetti ME. Drugs and falls in older people: a systematic review and meta-analysis. I. Psychotropic drugs. J Am Geriatr Soc 1999; 47: 30-9.

44. Leipzig RM, Cumming RG, Tinetti ME. Drugs and falls in older people: a systematic review and meta-analysis. II. Cardiac and analgesic drugs. J Am Geriatr Soc 1999; 47: 40-50.

45. Schoor NM van, Smit JH, Pluijm SMF, Jonker C, Lips P. Different cognitive functions in relation to falls among older persons. Immediate memory as an independent risk factor for falls. J Clin Epidemiol 2002; 55: 855-62.

46. Buchner DM, Larson EB. Transfer bias and the association of cognitive impairment with falls. J Gen Intern Med 1988; 3: 254-9.

47. Janti PO, Pyykko VI, Hervonen AL. Falls among elderly nursing home residents. Public Health 1993; 107: 89-96.

48. Rubenstein LZ, Josephson KR, Robbins AS (1994). Falls in the nursing home. Ann Intern Med 1994; 121: 442-451.

49. Morris J, Rubin E, Morris E, Mandel S. Senile dementia of the Alzmeimer's type: an important risk factor for serious falls. J Gerontol A Biol Sci Med Sci 1987; 42: 412-17. 


\section{Chapter 1}

50. Ott A, Breteler MM, van Harskamp F, Claus JJ, van der Cammen TJ, Grobbee DE, Hofman A. Prevalence of Alzheimer's disease and vascular dementia: association with education> The Rotterdam study. BMJ 1995 Apr 15; 310(6985): 970-3.

51. Sattin RW, Rodriguez JG, DeVito CA, Wingo PA. Home environmental hazards and the risk of fall injury among community-dwelling older persons. Study to Assess Falls Among the Elderly (SAFE) Group. J Am Geriatr Soc 1998; 46: 669-76.

52. Stalenhoef P, Diederiks J, Knottnerus A, de Wiite L, Crebolder H. How predictive is a homesafety checklist of indoor fall risk for the elderly living in the community? Eur J Gen Pract 1998; 46: 114-20.

53. Connell BR, Wolf SL> Environmental and behavioural circumstances associated with falls at home among healty elderly individuals. Atlanta FICSIT Group. Arch Phys Med Rehabil 1997 Feb; 78(2): 179-86.

54. Brody E, Kleban M, Moss M, Kleban F. Predictors of falls among institutionalised women with Alzheimer's disease. J Am Geriatr Soc 1984; 32: 877-82.

55. Buchner D, Larson E. Falls and fractures in patients with Alzheimer-type dementia. JAMA 1987; 257: 1492-95.

56. Steinberg M, Lyketsos CG, Steele C, Baker L, Brandt J, Baker A. Falls in the Institutionalized Elderly with Dementia: a pilot study. Nursing Home Medicine. The Annals of Long-Term Care.

57 Evans LK, Strumpf NE, Allen Taylor SL, Capezutti E, Maislin G, Jacobsen B. A clinical trial to reduce restraints in nursing homes. J Am Geriatr Soc 1997; 45: 675-81.

58 Capezutti E, Strumpf N, Evans L, Maislin G. Outcomes of nighttime physical restraint removal for severely impaired nursing home residents. Am J Alzheimer's Disease 1999; 14: 157-64.

59 Neufeld RR, Libow LS, Foley WJ, Dunbar JM, Cohen C, Breuer B. Restraint reduction reduces serious injuries among nursing home residents. J Am Geriatr Soc 1997; 47: 1202-7.

60. Morse JM. Preventing patient falls. Saga Publications, Inc. 1997.

61. Gillespie LD, Gillespie WJ, Robertson MC, Lamb SE, Cumming RG, Rowe BH. Interventions for Preventing Falls in Elderly People (Cochrane Review). In: The Cochrane Library, issue 2, 2004.

62. Perell KL, Nelson A, Goldman RL, Luther SL, Prieto-Lewis N, Rubenstein LZ. Fall risk assessment measures: an analytic review. Journal of Gerontology: Medical Sciences 2000; 56: M761-M766.

63. Moreland. A meta-analysis of fall prevention programs for the elderly: how effective are they? Nursing Research 2002; 51(1): 1-8.

64. Chang JT, Morton SC, Rubenstein et al. Interventions for the prevention of falls in the older adults: systemic review and meta-analysis of randomised clinical trials. BMJ 2004; 328: 680-686.

65. Ray WA, Taylor JA, Meador KG, Thapa PB, Brown AK, Kajihara HK et al. A randomized trial of a consultation service to reduce falls in nursing homes. JAMA 1997; 278: 557-562.

66. Feder G, Cryer C, Donovan S, Carter Y. Guideline for the prevention of falls in people over 65. BMJ 2000; 321: 1007-11. 
67. National Institute for Clinical Excellence. Falls: the assessment and prevention of falls in older people. November 2004.

68. Theune, Steultjens EM. Ergotherapierichtlijn Valpreventie. Nederlands Vereniging voor Ergotherapie 2005.

69. Schols JMGA, Crebolder HFJM, Weel C van. Nursing home and nursing home physician: the Dutch experience. J. Am Med Dir Assoc 2004; 5: 207-212.

70. Ribbe MW. Care for the elderly: the role of the nursing home in the Dutch Health care sysem. Int psychogeriatr 1993; 5: 213-22.

71. Robben BPM. Oudere chronische patiënten en hun woonvoorzieningen in de eerste helft van de twintigste eeuw. Tijdschr Gerontol Reriatr 1998; 29: 168-76.

72. Oogstvogels FJH. De historische ontwikkeling van het verpleeghuis. In: Trommel J, Ribbe MW, Stoop JA.(red), Capita Selecta van de Verpleeghyuisgeneeskunde. Utrecht/ Antwerpen, 1989: 3-19.

73. Schols JMGA (2000). In en vanuit het verpleeghuis: samenwerking verpleeghuisartshuisarts. Maastricht, academisch proefschrift Universiteit Maastricht.

74. Prismant \& Arcares. Nursing homes by numbers 2000: information from LZV and SIVIS. Utrecht 2002; Prismant/ Arcares.

75. Hoek JF, Ribbe MW, Hertogh CMPM, van der Vleuten CPM. The specialist training program for nursing home physicians: a new professional challenge. JAMDA 2001; 6: 326-30.

76. Hoek JF, Ribbe MW, Hertogh CMPM et al. The role of the specialist physician in nursing homes: the Netherlands. Int J Geriatr psych 2003; 18: 244-49.

77. Jongeneel F. Complexe zorg behoeft multidiscipl;inaire samenwerking. In Trommel J, Ribbe MW, Stoop JA.(red), Capita Selecta van de Verpleeghyuisgeneeskunde. Utrecht/Antwerpen, 1989: 115-21.

78. Hertogh CMPM, Deerenberg-Kessler W, Ribbe MW. The problem-oriented multidisciplinary approach in Dutch nursing home care. Clin Rehabil 1996; 10: 135-42.

79. Ribbe MW, van Mens JTh, Trijters DHM. Characteristics of patients during their stay in a nursing home and at discharge. Ned Tijdschr Geneeskd 1995; 139: 123-27.

80. SIVIS (National Nursing Home Registration System). Data verpleeghuizen 1986-1999. SIG-Zorginformatie, Utrecht.

81. Ribbe MW, Frijters DHM, Mens JTh van. Characteristics of nursing home patients at initial admission: age, sex and morbidity (in Dutch). Ned Tijdschr Geneeskd 1993; 137: 2544-48.

82. Boot JM, Knapen MHJM. Dutch Healthcare. Utrecht; Het Spectrum, 1996.

83. Baker SP, Harvey AH. Fall injuries in the elderly. Clin Geriatr Med 1985; 1: 501-12.

84. Van Schoor NM (2003). Prevention of hip fractures by external hip protectors. Amsterdam, academisch proefschrift Vrije Universiteit Amsterdam.

85. Ribbe MW, Ooms ME, Wal G van der, Eijk JThM. Wetenschappelijk onderzoek in de verpleeghuisgeneeskunde: een voorwaarde voor professionalisering. Ned Tidschr Geneeskd 1995; 139 (36): 1851-5. 



\section{Chapter 2}

\section{Falls in nursing homes: almost two per bed per year, $1.3 \%$ resulting in fracture}

B.P.J. Dijcks, J.C.L. Neyens, J.M.G.A. Schols, J.C.M. van Haastregt, H.F.J.M. Crebolder, L.P. de Witte. 


\section{AbStRACT}

\section{Objective}

To determine how many falls occur annually in Dutch nursing homes and how many result in fractures.

\section{Design}

Written questionnaire survey.

\section{Method}

All 371 Dutch nursing homes were asked to fill out a questionnaire on the number of somatic and psychogeriatric beds and the number of falls and fractures resulting from falls in 2000 and 2001.

\section{Results}

Of the 371 questionnaires, 202 (54\%) were returned. These were distributed as follows over the three types of nursing homes: combined: 151 (75\%), somatic: $15(7 \%)$, psychogeriatric: $36(18 \%)$. The average capacity of participating nursing homes was 180 beds. On average, there were more than 300 reported falls per nursing home per year: 336 in 2000 (SD: 180; median 314) and 311 in 2001 (SD: 165, median 294), which works out to almost 2 falls per bed per year. The number of falls per bed in the psychogeriatric patient group was higher than in the somatic patient group. There were an average of about 4 fallrelated fractures per year per nursing home: 4.3 in 2000 (SD: 3.7; median 4.0) and 3.6 in 2001 (SD: 2.8; median 3.0). The average number of annual fractures due to a fall was about 23 per 1000 beds. An average of $1.3 \%$ of falls resulted in fracture. In this respect, there were no clear differences between somatic and psychogeriatric patients.

\section{Conclusions}

The reported number of falls per nursing-home bed averaged almost 2 per year, and an average of $1.3 \%$ of these falls resulted in fracture. 


\section{INTRODUCTION}

A fall can be defined as 'an event which causes the patient to come unintentionally to the ground or some lower level, regardless of the cause'. ${ }^{1}$ Elderly people account for a fairly large number of fall incidents. Elderly nursing home residents have the biggest chance of falling by far, especially psychogeriatric patients. ${ }^{2}$ An international review based on the findings of 16 studies in nursing homes reported an annual average incidence of falls of 1.5 per bed. ${ }^{3}$ Two Dutch studies showed annual incidences of respectively 3.3 and 4.1 per psychogeriatric patient. ${ }^{4,5}$

It is not the high incidence of fall incidents that is alarming, but rather the combination of a high incidence with an increased risk of fall-related injuries in elderly nursing home residents. Fall incidents can have serious physical consequences, such as a hip fracture or other severe injury. Moreover, the mortality rate after a hip fracture is relatively high in nursing home residents. ${ }^{3}$ In the above-mentioned review, about $4 \%$ of fall incidents in nursing homes resulted in a fracture, $11 \%$ resulted in other injuries. ${ }^{3}$ The Dutch study in 1994 showed that $2.0 \%$ of all fall incidents resulted in a fracture and another $0.4 \%$ resulted in other serious physical injuries. ${ }^{4}$ The Dutch study in 1993 showed that $2.5 \%$ of fall incidents resulted in a fracture. ${ }^{5}$

Besides physical consequences, fall incidents may have psychological consequences, e.g. fear of falling, depression, perceived dependency and social isolation. ${ }^{3,6,7}$ These can have an important negative influence on people's quality of life. ${ }^{3}$ Falls often also increase the need for care in institutionalised patients, especially if it resulted in a hip fracture. In other words, falls also increase the workload of nursing staff.

There are no recent data in the Netherlands on the incidence of falls and fall-related fractures in nursing homes. The two Dutch studies mentioned above involved only a few nursing homes and may therefore not have been representative for all Dutch nursing homes. We conducted a survey among Dutch nursing homes to determine the number of fall incidents and the number of fall-related fractures in 2000 and 2001.

\section{Methods}

We composed a questionnaire that was sent to all nursing homes in the Netherlands. We used the Arcares database (National Branch Organisation for Nursing Homes and Old Peoples' Homes) to find the addresses of 371 nursing 
homes to which the questionnaires were sent. In an accompanying letter addressed to the management, we requested the care manager to complete the questionnaire. The compulsory national fall incidents registration system could be used to fill in the questionnaire. Three weeks after sending the questionnaires, we sent out a reminder. The questionnaire inquired about the number of somatic and psychogeriatric beds, and the number of fall incidents and fallrelated fractures in 2000 and 2001. We asked that data be given separately for the psychogeriatric and somatic wards, and for all wards together.

\section{ANALYSIS}

Data were analysed with the statistical computer programme SPSS (Statistical Package for the Social Sciences Benelux BV, Gorinchem). Frequencies were calculated and means and standard deviations were calculated for the discrete variables. Due to the skewed distribution of some outcomes, we calculated medians as well.

We calculated the number of fall incidents per bed in order to compare somatic and psychogeriatric patients, and to compare our data with the literature. The number of fractures was calculated per 1000 beds. Per nursing home, these numbers were calculated separately for the somatic and psychogeriatric beds, and for the total number of beds.

We calculated the number of fractures as a percentage of the number of fall incidents. In order to determine whether these data were representative for all nursing homes in the Netherlands, we compared the division of the different types of nursing homes in our study group with the national distribution which was: $72 \%$ combined nursing homes, $13 \%$ somatic nursing homes, and $14 \%$ psychogeriatric nursing homes. ${ }^{8}$

\section{RESULTS}

Response

A total of 371 questionnaires were sent and 202 were returned (54\%). The three types of nursing homes were represented as follows: 151 combined nursing homes $(75 \%), 15$ somatic nursing homes $(7 \%)$ and 36 psychogeriatric nursing homes $(18 \%)$. Somatic nursing homes were underrepresented compared with nationwide distribution, whereas both the combined and psychogeriatric nursing homes were slightly overrepresented in our study sample. 


\section{Number of beds}

The participating somatic nursing homes had a mean number of 124 beds (SD: 46), the psychogeriatric nursing homes had 136 (SD: 66), and the combined nursing homes had the largest capacity with a mean number of 197 beds (SD: 91). The latter had a larger number of psychogeriatric beds, on average, than somatic beds. We must point out that some nursing homes with large numbers of beds were actually organisations with different locations.

\section{Number of fall incidents}

The participating nursing homes, with a mean number of 180 beds, had over 300 reported fall incidents per nursing home per year; 336 incidents in 2000 (SD: 180; median 314), 311 incidents in 2001 (SD: 165; median 294). We present the number of fall incidents per bed for each category separately and for the entire group (see Table 1). In table 1, the number of somatic wards is the sum of somatic nursing homes and somatic wards in combined nursing homes. The number of psychogeriatric wards is the sum of psychogeriatric nursing homes and psychogeriatric wards in combined nursing homes.

The participating Dutch nursing homes had an average of almost 2 reported fall incidents per bed per year. The number of fall incidents per psychogeriatric bed was higher than that per somatic bed.

\begin{tabular}{|c|c|c|c|c|c|c|}
\hline \multirow{2}{*}{$\begin{array}{l}\text { Fall incidents } \\
\text { per bed }\end{array}$} & \multicolumn{2}{|c|}{ Somatic wards } & \multicolumn{2}{|c|}{ Psychogeriatric wards } & \multicolumn{2}{|c|}{ Total } \\
\hline & $\begin{array}{c}2000 \\
(n=105)\end{array}$ & $\begin{array}{c}2001 \\
(n=116)\end{array}$ & $\begin{array}{c}2000 \\
(n=126)\end{array}$ & $\begin{array}{c}2001 \\
(n=137)\end{array}$ & $\begin{array}{c}2000 \\
(n=176)\end{array}$ & $\begin{array}{c}2001 \\
(n=184)\end{array}$ \\
\hline \multicolumn{7}{|l|}{ number; $n(\%)$} \\
\hline$\leq 1.00$ & $41(39)$ & $42(36)$ & 6 (5) & 7 (5) & $17(10)$ & $18(10)$ \\
\hline $1.01-1.50$ & $33(31)$ & $43(37)$ & $14(11)$ & $22(16)$ & $44(25)$ & $59(32)$ \\
\hline $1.51-2.00$ & $19(18)$ & $14(12)$ & $25(20)$ & $32(23)$ & $41(23)$ & $34(18)$ \\
\hline $2.01-2.50$ & $4(4)$ & $10(9)$ & $29(23)$ & $27(20)$ & $43(24)$ & $43(23)$ \\
\hline $2.51-3.00$ & $3(3)$ & $3(3)$ & $31(25)$ & $24(18)$ & $19(11)$ & $19(10)$ \\
\hline$\geq 3.01$ & $5(5)$ & 4 (3) & $21(17)$ & $25(18)$ & $12(7)$ & $11(6)$ \\
\hline median & 1.3 & 1.2 & 2.4 & 2.2 & 1.9 & 1.8 \\
\hline mean (SD) & $1.3(0.6)$ & $1.3(0.6)$ & $2.3(0.9)$ & $2.2(0.8)$ & $1.9(0.7)$ & $1.8(0.6)$ \\
\hline
\end{tabular}

* some forms were incomplete 


\section{Chapter 2}

\section{Number of fall-related fractures}

There were almost 4 reported fall-related fractures per nursing home per year, on average: 4.3 in 2000 (SD: 3.7; median 4.0), 3.6 in 2001 (SD: 2.8; median 3.0). The number of fall-related fractures per 1000 beds per year was about 23 (see Table 2). The group of psychogeriatric patients had more fractures per bed than the group of somatic patients. The average rate of fall-related fractures, as a percentage of the number of fall incidents, was $1.3 \%$. In this respect, there were no clear differences between somatic and psychogeriatric patients.

\begin{tabular}{|c|c|c|c|c|c|c|}
\hline \multirow{2}{*}{$\begin{array}{l}\text { Fractures per } \\
1000 \text { beds }\end{array}$} & \multicolumn{2}{|c|}{ Somatic wards } & \multicolumn{2}{|c|}{ Psychogeriatric wards } & \multicolumn{2}{|c|}{ Total } \\
\hline & $\begin{array}{c}2000 \\
(n=85)\end{array}$ & $\begin{array}{c}2001 \\
(n=92)\end{array}$ & $\begin{array}{c}2000 \\
(n=106)\end{array}$ & $\begin{array}{c}2001 \\
(n=111)\end{array}$ & $\begin{array}{c}2000 \\
(n=158)\end{array}$ & $\begin{array}{c}2001 \\
(n=166)\end{array}$ \\
\hline \multicolumn{7}{|l|}{ number; $n(\%)$} \\
\hline 0 & $38(45)$ & $39(42)$ & $20(19)$ & $17(15)$ & $20(13)$ & $14(8)$ \\
\hline $0.01-20.00$ & $29(34)$ & $32(35)$ & $23(22)$ & $27(24)$ & $58(37)$ & $72(43)$ \\
\hline $20.01-40.00$ & $13(15)$ & $14(15)$ & $37(35)$ & $36(32)$ & $51(32)$ & $57(34)$ \\
\hline$>40.00$ & $5(6)$ & 7 (8) & $26(25)$ & $31(28)$ & $29(18)$ & $23(14)$ \\
\hline median & 8.3 & 9.5 & 24.4 & 26.7 & 21.0 & 19.6 \\
\hline mean (SD) & $10.9(13.1)$ & $12.2(15.5)$ & $26.3(20.5)$ & $28.8(22.2)$ & $22.8(17.6)$ & $22.7(16.7)$ \\
\hline
\end{tabular}

* some forms were incomplete

\section{Discussion}

This study showed that a considerable number of fall incidents occur in nursing homes, with an average of almost two falls per bed per year. With regard to nursing home patients, the literature showed that the high incidence rate is often caused by walking disorders, general weakness, dizziness, chronic diseases, cognitive problems, confusion and problems with activities of daily living, which all increase the fall risk. 3,9,10

Psychogeriatric patients fall more often than somatic patients. This study however showed a lower fall incidence rate for this group than two other previous Dutch studies. ${ }^{4,5}$ These lower numbers may be explained in part by difference in study population: one study ${ }^{4}$ excluded immobile patients, the other study ${ }^{5}$ included only patients with dementia. Furthermore, Van Dijk et al. obtained data from only one nursing home ${ }^{5}$, while in the Vermeulen study, data 
were obtained from 9 nursing homes. ${ }^{4}$ Therefore, these two studies were not representative for all Dutch nursing homes.

Another explanation for the differences with these previous studies could be the increase in attention for fall prevention in nursing homes in the past few years.

According to several researchers, the higher fall rate among psycho-geriatric patients can be attributed to the frequent occurrence of cognitive problems, behavioural problems and additional morbidity within this group. ${ }^{4,5,11}$ Over $80 \%$ of these patients suffer from dementia. ${ }^{12}$ The deteriorating locomotive functioning of dementia patients can lead to complete immobility. During this process, the risk of falling increases. These patients might also have a reduced awareness of the risks of falling. Finally, psychogeriatric patients had a mean higher age than somatic patients, which might also explain the higher fall risk. $^{13,14}$

The questionnaire showed that an average of $1.3 \%$ of falls resulted in fractures. This percentage was lower than the numbers mentioned in previous studies. Perhaps this could be attributed to the increased use of protective aids, e.g. hip protectors, which decrease the risk of a hip fracture. Better prevention/ treatment of osteoporosis could play a role as well, e.g. taking calcium, vitamin $\mathrm{D}$ and biphosphonates.

There are some remarks to take into account when interpreting our findings. We emphasise that this study only gives the number of reported falls and fallrelated fractures. Although nursing homes are obliged to register all incidents, the real number of fall incidents and fractures may be higher due to underreporting. Secondly, the group of somatic homes was slightly underrepresented compared to the national situation, while the combined and psychogeriatric homes were slightly overrepresented. This could mean that the actual number of fall incidents per nursing home in the Netherlands might be lower than the numbers obtained in this survey. Furthermore, it is possible that homes which returned the questionnaire had a more active fall prevention policy, in which case we might have found higher incidence numbers if the data of all nursing homes had been available. Data on fall prevention policy, however, were not available for either the responding homes or from the homes that did not respond. We do know that less than $5 \%$ of the responding nursing homes had a fall prevention protocol. ${ }^{17}$ Although the lack of (written) fall prevention protocols does not necessarily mean that no fall prevention activities are applied, it does indicate more or less that nursing homes have inadequate policy on fall prevention. The chance that the results would lead to misrepresentation is therefore rather small. A strong characteristic of the present 


\section{Chapter 2}

study is the response rate, which was above the average for the applied research method and which was enough to produce reliable conclusions.

Our research shows that falls in nursing homes are a serious problem. We therefore recommend the development and implementation of fall prevention interventions. Due to the relatively high risk of falls and fall-related morbidity in psychogeriatric patients, it is important that extra attention is given to fall prevention in this group of patients.

Prof. Dr. W.J.A. van den Heuvel, medical sociologist, Maastricht University, contributed to this article. 


\section{References}

1. Kellogg International Work Group on the prevention of falls by the elderly. The prevention of falls in later life. Dan Med Bull 1987; 34: 1-24.

2. Vermeulen HBM. Vallen en valpreventie bij psychogeriatrische verpleeghuisbewoners. In: Het verpleeghuis thuis? Nederlandse Vereniging van verpleeghuisartsen, 1988.

3. Rubenstein LZ, Josephson KR, Robbins AS. Falls in the nursing home. Ann Intern Med 1994;121:442-51.

4. Vermeulen HBM. Vallen en valpreventie in het verpleeghuis: een interventiestudie naar het effect van een bed-alarm-systeem. Nijmegen, academisch proefschrift Katholieke Universiteit Nijmegen 1994.

5. Dijk PTM van, Meulenburg OGRM, Sande HJ van de, Habbema JDF. Falls in dementia patients. Gerontologist 1993;33:200-4.

6. American Geriatrics Society, British Geriatrics Society \& American Academy of Orthopaedic Surgeons on Falls Prevention. Guideline for the prevention of falls in older persons. J Am Geriatr Soc 2001;49:664-72.

7. Tinetti ME, Mendes de Leon CF, Doucette JT, Baker DI. Fear of falling and fall-related efficacy in relationship to functioning among community living elders. J Gerontol 1994;49:M140-7.

8. Bartels LP. Instellingen van intramurale gezondheidszorg. Basisgegevens per 1-1-2002. Utrecht: Prismant, 2002.

9. Bedsine RW, Rubenstein LZ, Snyder L, editors. Medical Care of the Nursing Home Resident. Philadelphia PA. American College of Physicians, 1996.

10. Ejaz FK, Jones JA, Rose MS. Falls among nursing home residents: An examination of incident reports before and after restraint reduction programs. Journal of the American Geriatrics Society 1994;42:960-4.

11. Tinetti ME, Speechley M, Ginter SF. Risk factors for falls among elderly persons living in the community. N Engl J Med 1988;319:701-7.

12. SIG Informatiecentrum voor de gezondheidszorg (SIG). Jaarboek verpleeghuizen 1989. Utrecht: SIG; 1990.

13. Verpleeghuizen in cijfers 2000. Informatie uit LVZ en SIVIS. Utrecht: Prismant; 2000.

14. Bueno-Cavanillas A, Padilla-Ruiz F, Jiménez-Moleón JJ, Peinado-Alonso CA, GálvezVargas R. Risk factors in falls among the elderly according to extrinsic and intrinsic precipitating causes. Eur J Epidemiol 2000, 16: 849-859.

15. Chel VGM, Ooms ME. Fractuurpreventie bij ouderen in het verpleeghuis: valpreventie, vitamine D en heupbeschermers. Tijdschrift voor Verpleeghuisgeneeskunde 1999;23: 25-9.

16. Meyer G, Warnke A, Bender R, Mühlhauser I. Effect on hip fractures of increased use of hip protectors in nursing homes: cluster randomised controlled trial. BMJ 2003;326:76-8.

17. Dijcks BPJ, Neyens JCL. Valincidenten in verpleeghuizen 2000-2001. Hoensbroek: iRv; 2002. 



\section{Chapter 3}

Effects and feasibility of interventions for preventing falls in elderly people in long-term care facilities: systematic review

J.C.L. Neyens, J.C.M. van Haastregt, B.P.J. Dijcks, W.J.A. van den Heuvel, L.P. de Witte, J.M.G.A. Schols. 


\section{AbStRACT}

\section{Objectives}

To assess the effectiveness and feasibility of interventions aimed at reducing falls and related injuries in elderly residents in long-term care facilities.

\section{Design}

Systematic review.

\section{Data sources}

Database search covering MEDLINE, EMBASE and CINAHL.

\section{Review methods}

Randomised controlled trials that assessed falls and/or fall related injuries among elderly residents in long-term care facilities, were included. Two independent reviewers extracted data on trial characteristics, intervention characteristics, outcomes, and feasibility aspects of the intervention.

\section{Results}

Thirteen trials met our inclusion criteria. Four of these trials were found to have significant favourable effects on falls and/or injurious falls. The effective programmes were: comprehensive structured individual assessment with specific safety recommendations; a multidisciplinary programme including both general strategies and strategies tailored specifically to residents; a multifaceted nonpharmaceutical intervention; and calcium plus vitamin D supplementation.

\section{Conclusions}

Due to the limited and inconsistent evidence available, we could not formulate a definite conclusion about which types of programmes effectively reduce falls and related injuries among residents in long-term care facilities. A careful approach is needed as programmes to prevent falls in long-term care facilities may even have adverse effects. This may occur when a programme is not feasible for the setting in which it is implemented. We therefore recommend that health care professionals who wish to reduce falls among persons in longterm care facilities develop tailor-made interventions for their specific setting and population. These interventions should preferably be based on the four programmes which were found to have positive effects.

\section{Key Words}

accidental falls; fallers; prevention; older people; long-term care 


\section{INTRODUCTION}

The aim of the present study is to assess the effectiveness and feasibility of interventions aimed at reducing falls and related injuries in elderly residents in long-term care facilities. Fall incidents occur frequently in this group, especially among patients with cognitive impairments. ${ }^{1-9}$ Fall incidents may have considerable negative consequences. Approximately $10 \%$ of falls result in fractures ${ }^{10-14}$, and $25 \%$ of persons sustaining a hip fracture die within a year after the fall. ${ }^{15}$

There is extensive literature on interventions to prevent or reduce falls in the elderly $^{16-21}$, targeting elderly persons living in the community as well as residents in long-term care facilities and hospitalised elderly. Gillespie et al. carried out a systematic review of such fall interventions. ${ }^{16}$ They found that various interventions reduced the incidence of falls, including multidisciplinary interventions targeting multiple risk factors, and strengthening and balance exercises prescribed by a trained health professional and done at home. Tai Chi and home hazards assessment and modification by a health professional might also reduce falls. Gillespie et al. also found that individually tailored interventions delivered by a health professional were more effective than standard or group delivered programmes. These findings, however, were based mainly on studies of communityliving persons. Out of a total of 62 studies selected, only eight trials concerned persons in long-term care facilities. This group differs considerably from the population of elderly people living in the community who are relatively healthy. Likewise, a long-term residential care setting cannot be compared with a community setting. It is important, therefore, to systematically assess the effectiveness of interventions to prevent falls and related injuries specifically for residents in long-term care facilities. A number of new trials into fall prevention in long-term care facilities carried out in the last three years also warrant a separate systematic review.

Systematic reviews generally give little information about feasibility aspects of interventions, such as the extent to which interventions were implemented according to plan, compliance of the target population, and possible obstacles in the implementation process. In our opinion, insight into the feasibility of interventions is essential to a good interpretation of the outcome results. ${ }^{22}$ In the present paper we therefore combined a systematic review of the effectiveness of interventions aimed at reducing falls and related injuries in long-term care with a systematic review of the reported feasibility of these interventions. The advantage of assessing both effectiveness and feasibility is twofold. If there are no favourable effects, information about the feasibility of the interventions may help us 
distinguish between interventions that were ineffective (i.e. based on the wrong concept or theory) and interventions that were poorly implemented. ${ }^{22,23}$ If there are favourable effects, insight into feasibility is important because a promising intervention can only be implemented successfully in regular care when it is feasible for performers and patients.

\section{Methods}

\section{Search strategy}

Gillespie et al. performed an extensive and sensitive search on interventions for preventing falls in elderly people, covering MEDLINE (1966 to February 2003), EMBASE (1988 to 2003 week 19) and CINAHL (1982 to April 2003) and producing 62 trials. ${ }^{16}$ Of these, we selected all papers concerning elderly residents in longterm care facilities. We also extended the search to the first half of 2006 using the same search procedure as Gillespie et al., i.e. the optimum trial search strategy recommended by the Cochrane Collaboration combined with the search terms: accidental falls, falls, or fallers and aged, older, senior, or elderly. ${ }^{16}$ We also conducted a hand search of reference lists of the selected papers.

In order to optimise our assessment of the interventions' feasibility, we searched MEDLINE (1966 to May 2006), EMBASE (1988 to May 2006), and CINAHL (1982 to May 2006) for additional publications about the included trials. We looked for information about the following feasibility aspects: a) the extent to which the intervention was implemented according to plan; b) the compliance of the target population with the intervention; and c) possible obstacles in the implementation process. For this search, we used the surname of each of the authors of the main paper combined with the search terms: accidental falls, falls or fallers and aged, older, senior, or elderly.

\section{Selection criteria}

Trials were included when:

a. the effect of the intervention on falls and/or fall-related injuries had been assessed;

b. the trial had been performed among elderly residents in long-term care facilities.

\section{Data extraction}

First, three reviewers $(\mathrm{JN}, J \mathrm{~S}, \mathrm{JvH})$ independently reviewed the abstracts resulting from the search (2003-2006). Full texts were obtained when at least 
two reviewers considered the paper to be potentially relevant. The full texts were subsequently checked by two reviewers (JN and JS, or JN and JvH) to assess whether the papers did indeed meet our inclusion criteria. In addition two reviewers (JN and JS) assessed which of the 62 trials in the review by Gillespie et al. met our inclusion criteria. For both procedures, disagreements were resolved by consensus or third party adjudication. This resulted in a final selection of trials.

We then searched for additional papers about the included trials. The abstracts obtained through this search were checked by two reviewers (JN and JS). Full texts were obtained when papers appeared to refer to the included trials and these were again checked by two reviewers (JN and JS). Papers, which contained additional information on the included trials, were included in our review.

Two reviewers (either JN and JS, or JN and JvH) independently collected data from all included papers. The reviewers had developed a data extraction form for this purpose and tested it prior to use. Data were extracted on trial characteristics, intervention characteristics, and fall-related outcomes. With regard to the last, the reviewers noted, for each trial, the rate of falls and the rate of falls resulting in injury. If these data were not given, the reviewers noted data on related fall outcomes, if available. The statistical significance of differences between the groups was noted and, if available, measures of association. In addition, we extracted data on the feasibility aspects mentioned above (implementation according to plan; compliance of target population; obstacles during implementation). We also registered the implications for practice as reported by the authors of the papers. Disagreements were resolved by consensus or third party adjudication.

\section{ResUlts}

The eight trials in Gillespie's selection, which were carried out in long-term care facilities, met our inclusion criteria and were included in this review. Our additional search of the period from 2003 to May 2006 produced 1345 hits (see figure 1). We considered 32 papers to be potentially relevant on the basis of their abstracts and retrieved the full texts. Following a thorough screening, we included five additional trials, making a total of 13 trials in the present review. $^{24-38}$

All 13 papers contained some information about the interventions' feasibility. The search for additional papers reporting on feasibility produced only one relevant paper concerning a trial by Schnelle et al. ${ }^{39}$ 


\section{Chapter 3}

Figure 1. Search for relevant trials and additional papers
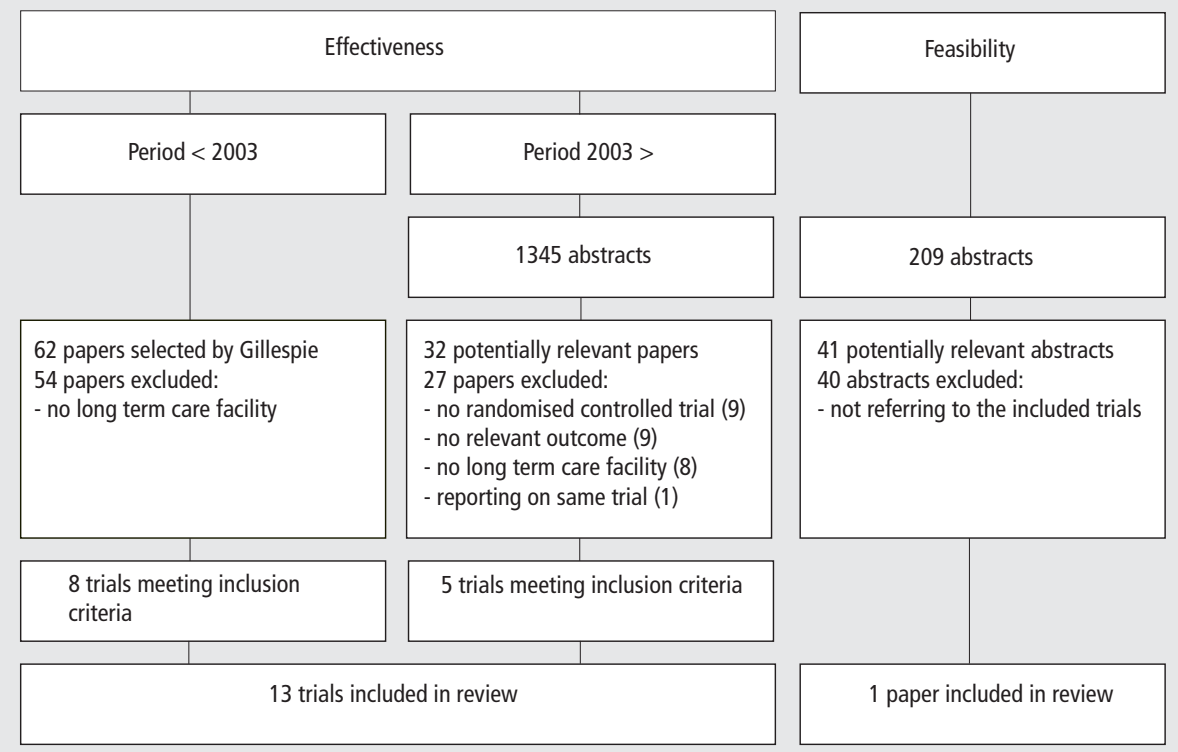

41 potentially relevant abstracts 40 abstracts excluded:

- not referring to the included trials

- no relevant outcome (9)

- no long term care facility (8)

reporting on same trial (1)

5 trials meeting inclusion criteria

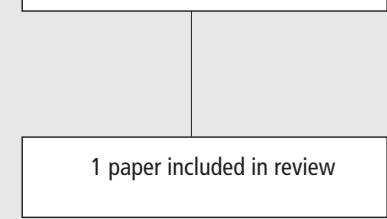

The first column of table 1 contains general descriptions of the intervention programmes. Table 2 contains more detailed information about programme characteristics. The majority of programmes were multifactorial $(n=10)$ and multidisciplinary $(n=8)$. In eight programmes, some form of assessment preceded the actual intervention. Nine programmes were individually tailored. Common programme elements were: exercise/physical therapy $(n=9)$, staff education $(n=8)$, improving environmental and personal safety $(n=8)$, resident education $(n=5)$, medication change $(n=7)$, and referral to relevant disciplines $(n=6)$. Eleven of the thirteen programmes were primarily aimed at preventing falls and/or injuries. . $^{2,26-33,35-38}$

Table 1 provides general information about the characteristics and outcomes of programmes in the included trials. Four out of thirteen trials resulted in a significant reduction in either the fall rate $(n=2)^{31,32}$, the percentage of persons with recurrent falls $(n=1)^{26}$, or both the fall rate and the percentage of persons sustaining a fall with serious injury $(n=1) .{ }^{28-30}$ In one trial, the intervention had a significant adverse effect on fall rate. ${ }^{37}$

Programmes which had statistically significant favourable effects were: comprehensive structured individual assessment with specific safety recommen- 
dations ${ }^{26}$; a multidisciplinary programme including both general strategies and strategies tailored specifically to resident ${ }^{28-30}$; a multifaceted nonpharmaceutical intervention ${ }^{31}$; and calcium plus vitamin D supplementation. ${ }^{32}$ The programme which had adverse effects consisted of systematic, individualised fall risk management for all residents using a fall-risk assessment tool, high risk logo, and strategies to address identified risks. ${ }^{37}$ More details about these programmes are shown in table 2.

Table 3 provides information on the reported feasibility of the interventions in the included trials, as well as implications for practice as reported by the authors. The extent to which the intervention was implemented according to protocol was reported for only one trial. Some information on compliancerelated issues was available for all trials. Reported compliance ranged from low to good. Most studies mentioned a number of factors which may have reduced programme effectiveness, such as: low compliance rates, inability to target the most important risk factors, an insufficiently vigorous intervention, lack of support for the intervention among staff, the intervention was time consuming and may have kept staff members from their other duties, and the limited resources of contemporary long-term care. Implications for practice, given for all trials, included: improving compliance, intensifying the intervention, targeting the intervention to specific subgroups, and improving the quality of implementation by providing adequate resources and training. 


\section{Chapter 3}

Table 1. General programme characteristics and outcomes

\begin{tabular}{llllll}
\hline Study & Intervention programmes & Setting & Participants & \multicolumn{2}{c}{ Randomis } \\
& & & & & \\
\hline Rubenstein, & IG: Comprehensive post-fall & Long-term & Mean age 87; & IG & 79 \\
1990, & assessment followed by & residential & recently fallen & CG & 81 \\
USA $^{24}$ & recommendations & care facility & & &
\end{tabular}

CG: usual care

\begin{tabular}{|c|c|c|c|c|}
\hline \multirow{5}{*}{$\begin{array}{l}\text { Mulrow, } \\
1994, \\
\text { USA }^{25}\end{array}$} & IG: Individually tailored one-on-one & \multirow[t]{4}{*}{ Nursing home } & Mean age 80; & \\
\hline & physical therapy sessions 3 times & & dependent for at & CG \\
\hline & a week for 4 months & & least 2 activities & \\
\hline & & & of daily living & \\
\hline & CG: Friendly visits & & & \\
\hline
\end{tabular}

\begin{tabular}{|c|c|c|c|c|c|}
\hline $\begin{array}{l}\text { Ray, } \\
1997, \\
\text { USA }^{26}\end{array}$ & $\begin{array}{l}\text { IG: Comprehensive structured } \\
\text { individual assessment with specific } \\
\text { safety recommendations } \\
\text { CG: usual care }\end{array}$ & Nursing home & $\begin{array}{l}\text { Mean age 83; } \\
\text { residents at high } \\
\text { risk for falls who } \\
\text { had a problem in } \\
\text { a study safety domain }\end{array}$ & $\begin{array}{l}\text { IG } \\
\text { CG }\end{array}$ & $\begin{array}{l}221 \\
261\end{array}$ \\
\hline $\begin{array}{l}\text { McMurdo, } \\
2000, \\
\text { UK }^{27}\end{array}$ & $\begin{array}{l}\text { IG: 6-month falls risk factor } \\
\text { assessment/modification and } \\
\text { seated balance exercise } \\
\text { training (twice-weekly) } \\
\text { CG: } 6 \text { months of } \\
\text { reminiscence therapy }\end{array}$ & $\begin{array}{l}\text { Residential } \\
\text { home for } \\
\text { elderly } \\
\text { people }\end{array}$ & Mean age 84 & $\begin{array}{l}\text { IG } \\
\text { CG }\end{array}$ & $\begin{array}{l}77 \\
56\end{array}$ \\
\hline $\begin{array}{l}\text { Jensen, } \\
2002, \\
\text { Sweden } \\
\text { 28-30 }\end{array}$ & $\begin{array}{l}\text { IG: } 11 \text { week multidisciplinary } \\
\text { programme including both } \\
\text { general and resident-specific } \\
\text { tailored strategies }\end{array}$ & $\begin{array}{l}\text { Residential care } \\
\text { facility }\end{array}$ & $\begin{array}{l}\text { Median age 83; } \\
\text { disabled by } \\
\text { cognitive or } \\
\text { physical } \\
\text { impairment }\end{array}$ & $\begin{array}{l}\text { IG } \\
\text { CG }\end{array}$ & \\
\hline
\end{tabular}

CG: usual care 


\begin{tabular}{|c|c|c|c|c|c|}
\hline \multirow{2}{*}{$\begin{array}{l}\text { Follow-up } \\
\text { in months } \\
24\end{array}$} & \multirow{2}{*}{$\begin{array}{l}\text { Outcomes } \\
\text { fall rate per person per } 2 \text { years }\end{array}$} & \multicolumn{2}{|c|}{ IG versus CG* } & \multirow{2}{*}{$\begin{array}{l}\text { Measures of } \\
\text { association }\end{array}$} & \multirow{2}{*}{$\begin{array}{l}\text { Significant } \\
\text { effect } \\
\text { no }\end{array}$} \\
\hline & & 4.09 & 4.51 & & \\
\hline & $\%$ persons with fractures & 9 & 6 & & no \\
\hline & $\begin{array}{l}\% \text { persons with laceration that } \\
\text { required sutures }\end{array}$ & $2.5 \%$ & $2.5 \%$ & & no \\
\hline \multirow[t]{3}{*}{4} & no. of falls & 79 & 60 & & no \\
\hline & $\begin{array}{l}\% \text { falls resulting in } \\
\text { serious injury }\end{array}$ & $8.9^{\dagger}$ & $3.3^{\dagger}$ & & no \\
\hline & $\begin{array}{l}\% \text { falls requiring health } \\
\text { care utilisation }\end{array}$ & $16.5^{\dagger}$ & $11.7^{\dagger}$ & & no \\
\hline \multirow[t]{3}{*}{12} & $\begin{array}{l}\% \text { persons with } \\
\text { recurrent falls }\end{array}$ & 43.8 & 54.1 & & yes $(+)$ \\
\hline & serious injury rate & & & & \\
\hline & per 100 person years & 13.7 & 19.9 & & no \\
\hline \multirow[t]{2}{*}{12} & fall rate per person week & 0.058 & 0.074 & & no \\
\hline & $\%$ persons with fractures & 2 & 8 & & no \\
\hline \multirow[t]{4}{*}{8} & $\begin{array}{l}\text { fall rate per } 1000 \\
\text { person days }\end{array}$ & 6.7 & 8.3 & rate ratio: 0.60 & yes $(+)$ \\
\hline & $\begin{array}{l}\text { minor injury rate per } \\
10000 \text { person-days }\end{array}$ & $12.5^{\dagger}$ & $14.7^{\dagger}$ & & no \\
\hline & $\begin{array}{l}\text { moderate injury rate } \\
\text { per } 10000 \text { person days }\end{array}$ & $2.7^{\dagger}$ & $1.7^{\dagger}$ & & no \\
\hline & $\begin{array}{l}\% \text { persons with femoral } \\
\text { fractures }\end{array}$ & 1.6 & 6.1 & odds ratio: 0.23 & yes $(t)$ \\
\hline
\end{tabular}




\section{Chapter 3}

Table 1. General programme characteristics and outcomes

\begin{tabular}{llllll}
\hline Study & Intervention programmes & Setting & Participants & \multicolumn{2}{c}{ Randomised } \\
& & & & & \\
\hline Becker, & IG: Multifaceted, & Nursing home & Mean age 84 & IG & 509 \\
2003, & nonpharmaceutical & & & CG & 472 \\
Germany ${ }^{31}$ & intervention & & &
\end{tabular}

CG: usual care

\begin{tabular}{llllll}
\hline $\begin{array}{l}\text { Bischoff, } \\
\text { 2003, }\end{array}$ & $\begin{array}{l}\text { IG: calcium plus vitamin D } \\
\text { supplementation }\end{array}$ & $\begin{array}{l}\text { Long-stay } \\
\text { geriatric } \\
\text { care }\end{array}$ & $\begin{array}{l}\text { Mean age 85; } \\
\text { ability to walk } 3 \mathrm{~m} \text { mand } \\
\text { with or without }\end{array}$ & $\begin{array}{l}\text { IG } \\
\text { CG }\end{array}$ & 62 \\
& CG: calcium supplementation & & $\begin{array}{l}60 \\
\text { a walking aid }\end{array}$ & & \\
\hline Meyer, & IG: Single education session & Nursing & 70 years or & IG & 459 \\
2003, & for nursing staff, who then & homes & older; not & CG & 483 \\
Germany 33 & educated residents; provision of & & bedridden & &
\end{tabular}

CG: optimised usual care

\begin{tabular}{llllll}
\hline Schnelle, & IG: low-intensity, functionally & Nursing & Mean age 88; & IG & 92 \\
2003, & oriented exercise and & homes & incontinent; & CG & 98 \\
USA $^{34}$ & incontinence care provided every & & long-stay & & \\
& 2 hours from 8 a.m. to 4 p.m., & & residents & & \\
& 5 days a week for 8 months & & & &
\end{tabular}

CG: usual care 


\begin{tabular}{|c|c|c|c|c|c|}
\hline \multirow{2}{*}{$\begin{array}{l}\text { Follow-up } \\
\text { in months } \\
12\end{array}$} & \multirow{2}{*}{$\begin{array}{l}\text { Outcomes } \\
\text { fall rate per } 1000\end{array}$} & \multicolumn{2}{|c|}{ IG versus CG* } & \multirow{3}{*}{$\begin{array}{l}\text { Measures of } \\
\text { association } \\
\text { relative risk: } \\
0.55\end{array}$} & \multirow{2}{*}{$\begin{array}{l}\text { Significant } \\
\text { effect } \\
\text { yes (+) }\end{array}$} \\
\hline & & 1399 & 2588 & & \\
\hline & person years & & & & \\
\hline & hip-fracture rate per & 43 & 39 & relative risk: & no \\
\hline & 1000 person years & & & 1.11 & \\
\hline & non-hip fracture rate & 41 & 52 & relative risk: & no \\
\hline & per 1000 person years & & & 0.78 & \\
\hline \multirow[t]{2}{*}{3} & fall rate per & 0.034 & 0.076 & & yes $(+)$ \\
\hline & person week & & & & \\
\hline
\end{tabular}

\begin{tabular}{|c|c|c|c|c|c|}
\hline Mean 15 for & fall rate per person & 0.17 & 0.22 & & no \\
\hline IG and & month & & & & \\
\hline 14 for $C G$ & & & & & \\
\hline & $\%$ persons with & 4.6 & 8.1 & relative risk: & no \\
\hline & hip factures & & & 0.57 & \\
\hline & $\%$ persons with other & 7.6 & 6.6 & relative risk: & no \\
\hline & fractures & & & 1.15 & \\
\hline 8 & fall rate per 1000 & 12.7 & 20.5 & & no \\
\hline & & perso & & & \\
\hline & skin injury rate per & 3.9 & 7.3 & & not reported \\
\hline & 1000 person weeks & & & & \\
\hline & bone fracture rate per & 2.0 & 0.9 & & not reported \\
\hline & 1000 person weeks & & & & \\
\hline & other injury rate per & 0.5 & 1.4 & & not reported \\
\hline & 1000 person weeks & & & & \\
\hline
\end{tabular}




\section{Chapter 3}

Table 1. General programme characteristics and outcomes

\begin{tabular}{|c|c|c|c|c|c|}
\hline Study & Intervention programmes & Setting & Participants & Ran & mised \\
\hline $\begin{array}{l}\text { Van Schoor, } \\
2003, \\
\text { Netherlands }{ }^{35}\end{array}$ & $\begin{array}{l}\text { IG: External hip protector } \\
\text { plus written information on } \\
\text { bone health and } \\
\text { risk factors for falls. } \\
\text { CG: written information on bone } \\
\text { health and risk factors for falls }\end{array}$ & $\begin{array}{l}\text { Nursing homes } \\
\text { and apartment } \\
\text { houses and } \\
\text { homes for } \\
\text { the elderly }\end{array}$ & $\begin{array}{l}\text { Mean age 85; } \\
\text { low bone density; } \\
\text { a high risk } \\
\text { for falling }\end{array}$ & $\begin{array}{l}\text { IG } \\
\text { CG }\end{array}$ & $\begin{array}{l}276 \\
285\end{array}$ \\
\hline $\begin{array}{l}\text { Dyer, } \\
2004, \\
\text { UK }^{36}\end{array}$ & $\begin{array}{l}\text { IG: multifactorial fall prevention } \\
\text { programme including } 3 \text { months } \\
\text { gait-balance training, medication } \\
\text { review, environmental check, } \\
\text { staff education, podiatry } \\
\text { and optometry } \\
\text { CG: visits by research nurse to } \\
\text { ensure completion of fall records }\end{array}$ & $\begin{array}{l}\text { Residential } \\
\text { care homes }\end{array}$ & Mean age 87 & $\begin{array}{l}\text { IG } \\
\text { CG }\end{array}$ & $\begin{array}{l}102 \\
94\end{array}$ \\
\hline $\begin{array}{l}\text { Kerse, } \\
\text { 2004, } \\
\text { New Zealand }{ }^{37}\end{array}$ & $\begin{array}{l}\text { IG: systematic individualised } \\
\text { fall-risk management for } \\
\text { all residents using a fall- } \\
\text { risk assessment tool, high risk } \\
\text { logo, and strategies to address } \\
\text { identified risks. } \\
\text { CG: usual care }\end{array}$ & $\begin{array}{l}\text { Residential } \\
\text { care homes }\end{array}$ & Mean age 83 & $\begin{array}{l}\text { IG } \\
\text { CG }\end{array}$ & $\begin{array}{l}346 \\
271\end{array}$ \\
\hline $\begin{array}{l}\text { Ray, } \\
2006, \\
\text { USA }^{38}\end{array}$ & $\begin{array}{l}\text { IG: an intensive 2-day safety } \\
\text { training programme for } \\
\text { facility staff, focusing } \\
\text { on living space and personal } \\
\text { safety; wheelchairs, canes, } \\
\text { and walkers; psychotropic } \\
\text { drug use; and transferring } \\
\text { and ambulation. } \\
\text { CG: not reported }\end{array}$ & $\begin{array}{l}\text { Long-term } \\
\text { care } \\
\text { facilities }\end{array}$ & $\begin{array}{l}\text { Mean age 84; } \\
\text { not bedridden }\end{array}$ & $\begin{array}{l}\text { IG } \\
\text { CG }\end{array}$ & $\begin{array}{l}4932 \\
5626\end{array}$ \\
\hline
\end{tabular}




\begin{tabular}{|c|c|c|c|c|c|}
\hline \multirow{2}{*}{$\begin{array}{l}\text { Follow-up } \\
\text { in months } \\
\text { At least } 12\end{array}$} & \multirow{2}{*}{$\begin{array}{l}\text { Outcomes } \\
\mathrm{n} \text { of persons with } \\
\text { recurrent falls }\end{array}$} & \multicolumn{2}{|c|}{ IG versus CG* } & \multirow{2}{*}{$\begin{array}{l}\text { Measures of } \\
\text { association } \\
\text { risk ratio: } 0.87\end{array}$} & \multirow{2}{*}{$\begin{array}{l}\text { Significant } \\
\text { effect } \\
\text { no }\end{array}$} \\
\hline & & 100 & 119 & & \\
\hline & $\begin{array}{l}\text { hip fracture rate } \\
\text { per } 100 \text { person years }\end{array}$ & 5 & 5 & & no \\
\hline & $\mathrm{n}$ of other fractures & 16 & 14 & & no \\
\hline \multirow[t]{2}{*}{12} & $\begin{array}{l}\text { fall rate per } \\
\text { person-year }\end{array}$ & 2.2 & 4.0 & & no \\
\hline & $\begin{array}{l}\% \text { persons } \\
\text { with fractures }\end{array}$ & 3.9 & 3.2 & odds ratio: 1.23 & no \\
\hline
\end{tabular}

\begin{tabular}{|c|c|c|c|c|c|}
\hline \multirow[t]{3}{*}{12} & $\begin{array}{l}\text { fall rate per } \\
\text { person year }\end{array}$ & 4.1 & 2.3 & $\begin{array}{l}\text { incidence rate } \\
\text { ratio (IRR): } 1.34\end{array}$ & yes $(-)$ \\
\hline & $\begin{array}{l}\text { injurious fall rate } \\
\text { per person-year }\end{array}$ & 1.6 & 1.0 & IRR: 1.12 & no \\
\hline & $\begin{array}{l}\text { serious injury rate } \\
\text { per } 10 \text { person year }\end{array}$ & 0.9 & 1.8 & IRR: 1.14 & no \\
\hline \multirow[t]{4}{*}{12} & $\begin{array}{l}\text { injury rate per } 1000 \\
\text { person years }\end{array}$ & 106.0 & 99.5 & rate ratio: 0.98 & no \\
\hline & $\begin{array}{l}\text { hip fracture rate per } \\
1000 \text { person years }\end{array}$ & 33.2 & 33.0 & rate ratio: 0.88 & no \\
\hline & $\begin{array}{l}\text { other fracture rate per } \\
1000 \text { person years }\end{array}$ & 30.0 & 28.8 & rate ratio: 1.03 & no \\
\hline & $\begin{array}{l}\text { soft tissue injury rate } \\
\text { per } 1000 \text { person years }\end{array}$ & 42.8 & 37.8 & rate ratio: 1.05 & no \\
\hline
\end{tabular}




\section{Chapter 3}

Table 2. Detailed programme characteristics

\begin{tabular}{ccccccc}
\hline Programme elements & Rubenstein, & Mulrow, & Ray, & McMurdo, & Jensen, & Becker, \\
& 1990, & 1994, & 1997, & 2000, & 2002, & 2003, \\
& USA $^{24}$ & USA $^{25}$ & USA $^{26}$ & UK $^{27}$ & Sweden $^{28-30}$ & Germany
\end{tabular}

\begin{tabular}{|c|c|c|c|c|c|c|}
\hline Assessment & yes & yes & yes & yes & yes* & no \\
\hline $\begin{array}{l}\text { Comprehensive } \\
\text { geriatric assessment }\end{array}$ & + & - & - & + & + & - \\
\hline Transfer and ambulation & - & + & + & - & + & - \\
\hline Medication review & + & - & + & + & + & - \\
\hline $\begin{array}{l}\text { Environmental / personal } \\
\text { safety assessment }\end{array}$ & - & - & + & + & + & +- \\
\hline (Correct) use of aids & - & - & + & - & + & - \\
\hline Intervention & yes & yes & yes & yes & yes & yes \\
\hline $\begin{array}{l}\text { Exercise/ Physical } \\
\text { Therapy programme }\end{array}$ & + & + & + & + & + & + \\
\hline Staff education & - & - & + & - & + & + \\
\hline $\begin{array}{l}\text { Improving environmental / } \\
\text { personal safety }\end{array}$ & + & - & + & + & + & + \\
\hline Resident education & - & - & + & - & - & + \\
\hline Change in medication & + & - & + & + & + & - \\
\hline Referral to relevant disciplines & + & - & + & + & - & - \\
\hline Hip protector & - & - & - & - & + & + \\
\hline Supplying/repairing aids & - & - & + & - & + & - \\
\hline $\begin{array}{r}\text { Recom } \\
\text { for pri } \\
\text { ph }\end{array}$ & $\begin{array}{l}\text { mendations } \\
\text { imary care } \\
\text { hysician }\end{array}$ & & & & & \\
\hline
\end{tabular}

\begin{tabular}{|c|c|c|c|c|c|c|}
\hline Individually tailored $^{\dagger}$ & yes & yes & yes & yes & yes & no \\
\hline Multidisciplinary $^{\ddagger}$ & yes & no & yes & yes & yes & yes \\
\hline \multicolumn{7}{|c|}{+ element included in the programme } \\
\hline \multicolumn{7}{|c|}{ - element not included in the programme } \\
\hline \multicolumn{7}{|c|}{ * part of baseline assessment (also for control subjects) } \\
\hline \multicolumn{7}{|c|}{$\dagger$ yes $=$ individually tailored; no $=$ standard or group delivered } \\
\hline$=$ at least two di & discip & Ivec & $y$ & in & & \\
\hline
\end{tabular}


Systematic review

\begin{tabular}{|c|c|c|c|c|c|c|}
\hline $\begin{array}{c}\text { Bischoff, } \\
\text { 2003, } \\
\text { Switzerland }\end{array}$ & $\begin{array}{c}\text { Meyer, } \\
\text { 2003, } \\
\text { Germany }\end{array}$ & $\begin{array}{l}\text { Schnelle, } \\
\text { 2003, } \\
\text { USA }^{34}\end{array}$ & $\begin{array}{l}\text { Van Schoor, } \\
\text { 2003, } \\
\text { Netherlands }{ }^{35}\end{array}$ & $\begin{array}{l}\text { Dyer, } \\
2004, \\
\text { UK }^{36}\end{array}$ & $\begin{array}{c}\text { Kerse, } \\
\text { 2004, } \\
\text { New Zealand }{ }^{37}\end{array}$ & $\begin{array}{l}\text { Ray, } \\
2005, \\
\text { USA }^{38}\end{array}$ \\
\hline no & no & no & no & yes & yes & yes \\
\hline - & - & - & - & + & + & - \\
\hline - & - & - & - & - & + & + \\
\hline - & - & - & - & + & + & + \\
\hline- & - & - & - & + & + & + \\
\hline- & - & - & - & - & + & + \\
\hline yes & yes & yes & yes & yes & yes & yes \\
\hline- & - & + & - & + & - & + \\
\hline - & + & - & + & + & + & + \\
\hline - & - & - & - & + & - & + \\
\hline - & + & - & + & - & - & + \\
\hline+ & - & - & - & + & - & + \\
\hline- & - & - & - & + & + & + \\
\hline - & + & - & + & - & - & - \\
\hline - & - & - & - & - & - & + \\
\hline & & $\begin{array}{c}\text { Incontinence } \\
\text { care }\end{array}$ & & & $\begin{array}{l}\text { Unspecified } \\
\text { strategies }\end{array}$ & \\
\hline no & no & yes & no & yes & yes & yes \\
\hline no & no & not specified & no & yes & yes & yes \\
\hline
\end{tabular}




\section{Chapter 3}

Table 3. Feasibility of the intervention and implications for practice as reported by the authors

\begin{tabular}{|c|c|c|}
\hline Study & $\begin{array}{l}\text { Implementation of } \\
\text { intervention according to plan }\end{array}$ & $\begin{array}{l}\text { Feasibility } \\
\text { Compliance of target population } \\
\text { with intervention }\end{array}$ \\
\hline $\begin{array}{l}\text { Rubenstein, } \\
1990, \\
\text { USA }^{24}\end{array}$ & Not reported & $\begin{array}{l}\text { Physicians and other caregivers complied with } \\
\text { nearly } 62 \% \text { of the recommendations. } \\
\text { Physician compliance did not significantly } \\
\text { correlate with falls. }\end{array}$ \\
\hline $\begin{array}{l}\text { Mulrow, } \\
1994, \\
\text { USA }^{25}\end{array}$ & Not reported. & $89 \%$ of scheduled PT sessions were completed. \\
\hline $\begin{array}{l}\text { Ray, } \\
1997, \\
\text { USA }^{26}\end{array}$ & Not reported & $\begin{array}{l}63 \% \text { of observable safety interventions had } \\
\text { been implemented after } 3 \text { months. }\end{array}$ \\
\hline $\begin{array}{l}\text { McMurdo, } \\
2000, \\
\text { UK }^{27}\end{array}$ & Not reported & $\begin{array}{l}68 \% \text { completed the intervention period. } \\
60 \% \text { to } 94 \% \text { of the modifications recommended } \\
\text { were carried out. }\end{array}$ \\
\hline $\begin{array}{l}\text { Jensen, } \\
2002, \\
\text { Sweden }^{28-30}\end{array}$ & Not reported & $\begin{array}{l}\text { Both staff and residents were active participants. } \\
\text { Compliance with exercise regime was } 88 \% \\
\text { ( } 70 \text { out of } 80 \text { ). A total of } 72 \% \text { agreed to use } \\
\text { hip protectors ( } 34 \text { out of } 47 \text { ) }\end{array}$ \\
\hline $\begin{array}{l}\text { Becker, } \\
\text { 2003, } \\
\text { Germany }^{31}\end{array}$ & Not reported & $\begin{array}{l}72 \% \text { participated in resident education; } 25 \% \\
\text { participated in the exercise classes. Mean } \\
\text { number of exercise classes was } 33 \text {. A total of } \\
31 \% \text { decided to wear hip protectors of which } \\
68 \% \text { showed } 100 \% \text { adherence }\end{array}$ \\
\hline $\begin{array}{l}\text { Bischoff, } \\
2003, \\
\text { Swizerland }^{32}\end{array}$ & $\begin{array}{l}\text { All subjects received } \\
\text { standard intervention as } \\
\text { allocated }\end{array}$ & $\begin{array}{l}\text { Tablets were swallowed in the presence of the } \\
\text { study nurse to ensure compliance. A total of } \\
73 \% \text { completed the study }\end{array}$ \\
\hline $\begin{array}{l}\text { Meyer } \\
\text { 2003, } \\
\text { Germany }\end{array}$ & Not reported & $\begin{array}{l}\text { Protectors were used during } 54 \% \text { of falls in the } \\
\text { intervention group compared with } 8 \% \text { in the } \\
\text { control group }\end{array}$ \\
\hline
\end{tabular}




\section{Implications for practice}

Obstacles / problems encountered

Environmental changes had relatively

low compliance rates.
Routine use of laboratory tests,

electrocardiograms, and Holter monitoring is not recommended as part of the assessment, except when there is a clinical indication or suspicion.

Subjects complained of moderate muscle soreness at $7 \%$ of sessions. Interventions focusing on strength and mobility deficits may be most beneficial while other modalities such as range-of-motion exercises may have little to no benefit.

Inability to target the most important risk factors and partial implementation of Programmes that address the reported limitations could be even more effective.

safety recommendations

High drop-out rate.

The exercises might not have been vigorous enough.

Not reported
Risk factors for falls and hence targets for intervention may be different in institutionalised elderly people compared to community-dwelling elderly persons

Subgroup analyses revealed that the higher cognition group benefited more from the programme with regard to reduction of falls, but femoral fractures were reduced in the lower cognition group.

A matter of concern is the resident's freedom Staff, administrators, and resident general physicians probably
to decide whether or not to participate in any
of the intervention elements. Residents who time to learn the modified use of hip protectors and
other fall prevention measures

A matter of concern is the resident's freedom Staff, administrators, and resident general physicians probably
to decide whether or not to participate in any
of the intervention elements. Residents who time to learn the modified use of hip protectors and
other fall prevention measures

A matter of concern is the resident's freedom Staff, administrators, and resident general physicians probably
to decide whether or not to participate in any
of the intervention elements. Residents who time to learn the modified use of hip protectors and
other fall prevention measures were motivated to wear hip protectors reported a lack of staff support with personal care, dressing, and the supply of hip protectors. Two subjects reported increased constipation in the calcium plus vitamin $D$ group, which Recurrent fallers seem to benefit most with calcium plus vitamin D supplementation did not lead to discontinuation of treatment Not reported Long term implementation of the intervention requires the provision of hip protectors on prescription for elderly people at high risk of hip fracture 


\section{Chapter 3}

Table 3. Feasibility of the intervention and implications for practice as reported by the authors

\begin{tabular}{|c|c|c|}
\hline Study & $\begin{array}{l}\text { Implementation of } \\
\text { intervention according to plan }\end{array}$ & $\begin{array}{l}\text { Feasibility } \\
\text { Compliance of target population } \\
\text { with intervention }\end{array}$ \\
\hline $\begin{array}{l}\text { Schnelle, } \\
2003, \\
\text { USA }^{34}\end{array}$ & Not reported & $\begin{array}{l}\text { Intervention group residents completed on } \\
\text { average } 3.2 \text { episodes of the programme per } \\
\text { day, of the } 4 \text { that were attempted each day } \\
\text { Residents' refusal and participation in a social } \\
\text { activity were the primary reasons that the } \\
\text { programme could not be performed completely } \\
\text { on any particular day. }\end{array}$ \\
\hline $\begin{array}{l}\text { Van Schoor, } \\
\text { 2003, } \\
\text { Netherlands }^{35}\end{array}$ & Not reported & $\begin{array}{l}\text { Compliance with hip protectors was } 61 \% \text { after } \\
1 \text { month, } 45 \% \text { after } 6 \text { months and } 37 \% \text { after } \\
12 \text { months. } 16 \% \text { reported using the hip } \\
\text { protectors at night. }\end{array}$ \\
\hline $\begin{array}{l}\text { Dyer, } \\
2004, \\
\text { UK }^{36}\end{array}$ & Not reported & $\begin{array}{l}81 \% \text { of the subjects in the intervention group } \\
\text { participated in the exercises. They attended } 59 \% \\
\text { of the sessions with a median of } 22 \text { sessions. } \\
\text { Medical assessments were conducted in } 70 \% \\
\text { of the cases. }\end{array}$ \\
\hline $\begin{array}{l}\text { Kerse, } \\
\text { 2004, } \\
\text { New Zealand }{ }^{37}\end{array}$ & Not reported & $\begin{array}{l}\text { Five of the } 7 \text { homes had high compliance with } \\
\text { the programme, assessing } 48 \text { to } 85 \% \text { of all } \\
\text { residents. The two remaining homes, termed } \\
\text { non-compliant homes, assessed } 0 \% \text { to } 35 \% \text { of } \\
\text { residents. Individualised assessments covered } \\
49 \% \text { of residents overall. An average of } 10 \\
\text { individual fall prevention strategies was } \\
\text { recommended. }\end{array}$ \\
\hline $\begin{array}{l}\text { Ray, } \\
2006, \\
\text { USA }^{38}\end{array}$ & Not reported & $\begin{array}{l}26 \% \text { complied with less than } 34 \% \text { of } \\
\text { recommendations, } 26 \% \text { complied with } \\
34 \%-66 \% \text {, and } 48 \% \text { complied with more than } \\
66 \%\end{array}$ \\
\hline
\end{tabular}




\section{Implications for practice}

Obstacles / problems encountered

The programme was performed by research staff, but this been done by nursing home staff, it would not have been according to protocol because staffing requirements for the programme exceed the staffing resources in most nursing homes.
Increasing the intensity of incontinence care or combining the intervention with optimal medical management may strengthen the intervention.

Fundamental changes in the staffing of most nursing homes will be necessary to translate such intervention into everyday practice.
Low compliance at night and lower impact effectiveness than expected.

The number of hip protectors ( 4 or 5 ) provided was not always sufficient Certain recommendations made to the residents/care homes, such as medication withdrawal, were not optimally followed up.
Compliance at night might increase with a more comfortable protector. Another possibility is to combine the hip protector with other interventions, such as a movement sensor; a softer hip protector during the night; or fall prevention strategies. Further intervention targeting only those at high risk should be considered
Two homes were non-compliant, but excluding them from the analysis did not alter the result for falls or injurious falls. The work of the fall-coordinator was time consuming and may have kept that staff member away from other duties.
To ensure programme implementation, adequate resources and training must be made available.

Higher activity levels of residents may explain the observed increase in falls.

In future research, some measurement of cognition will be needed and specific interventions for demented residents need to be further developed.

Any intervention that requires extra work and does not add extra staff is unlikely to have a positive effect.

The present study sought to train long-term Without further resources for facilities it will be difficult at present care facility staff to perform the assessment and subsequent interventions, which may have been infeasible in the constrained resources environment of contemporary long-term care to implement more intensive training programmes. It may also be important to place training programmes within the context of a general, structured quality improvement initiative. 


\section{DisCUSSION}

Four of the thirteen trials were found to have significant favourable effects on falls and/or injurious falls. ${ }^{26,28-32}$ The effective programmes were: comprehensive structured individual assessment with specific safety recommendations; a multidisciplinary programme including both general strategies and strategies tailored specifically to residents; a multifaceted nonpharmaceutical intervention; and calcium plus vitamin D supplementation.

The majority $(n=8)$ of programmes included in this review were multifactorial and multidisciplinary, as well as individualised. ${ }^{24-30,36-38}$ However, only three had favourable effects ${ }^{26,28-30,31}$ and one even had adverse effects. ${ }^{37}$ Remarkably, evaluations of two almost identical programmes, by Ray et al. ${ }^{38}$, in 1997 and 2005, gave contrary results. While the programme evaluated in 1997 showed significant favourable effects, no favourable effects at all were found in an evaluation in 2005. This inconsistency might have been caused by major differences in programme implementation. In 1997 the programme had been implemented directly in the participating settings, but in 2005 it was implemented indirectly, by means of an intensive two-day safety training programme for facility staff. They then had to implement the actual intervention themselves. Ray concluded that the programme as implemented in 2005 might have been infeasible because of the constrained resources in contemporary long-term care. ${ }^{38}$ This emphasises the importance of combining effectiveness studies with feasibility studies (process evaluations) in the field of fall prevention. An intervention which may be effective in itself might not yield favourable effects when it is not implemented according to plan, is badly complied with, and/or encounters serious obstacles in daily practice. In other words, information about the intervention process is essential for a good interpretation of trial results.

This review may have some limitations. First, we did not assess the trials' methodological quality. However, considering the limited number of studies available and the fact that we only included randomised controlled trials, a quality assessment would have added little value to the present results.

Second, we did not pool the data of the included trials because of the heterogeneity of the programmes, settings and participating populations. We might therefore have underestimated the effects of the programmes on falls resulting in injury. Most studies were underpowered to detect significant effects on injurious falls because power calculations were apparently based on the expected effect on falls. In six of the eleven trials, which included an assessment of the effect on injurious falls, the control group scored better than the intervention 
group. Although these were minor differences, it is unlikely that a higher power would have revealed more significantly favourable effects on injurious falls.

Third, it is possible that we overlooked relevant studies, in particular publications about the programmes' feasibility. This type of information is not always published in official scientific journals and might therefore have been unavailable to international readers.

\section{IMPLICATIONS}

Due to the limited and inconsistent evidence available, we could not formulate a definite conclusion about which types of programmes effectively reduce falls and related injuries among residents in long-term care facilities. A careful approach is needed as programmes to prevent falls among persons in long-term care facilities may even have adverse effects. This may occur when a programme is not feasible for the setting in which it is implemented. As Kerse, author of the negative trial noted: "Any intervention that asks for extra work and does not add extra staff is unlikely to have a positive effect" ${ }^{37}$ We therefore recommend that health care professionals who wish to reduce falls among persons in long-term care facilities develop tailor-made interventions for their specific setting and population. These interventions should preferably be based on the four programmes, which were found to have positive effects. In our opinion, it is extremely important that tailor-made intervention programmes are first tested on a small scale in order to assess their feasibility for a particular setting and to improve programme weaknesses. Special attention must go to actively monitoring correct implementation, improving compliance, and safeguarding the availability of human and material resources.

We can further enhance our knowledge of the effectiveness of interventions aimed at reducing falls and injurious falls among elderly residents in long-term care facilities by also evaluating their implementation (preferably in randomised controlled trials). These evaluations should include close examination of the intervention process, to determine programmes' practical feasibility. Process evaluations should always address the extent to which the intervention was performed according to protocol, compliance of target population with the intervention, and any possible obstacles in the implementation process. 
Chapter 3 


\section{References}

1. Perry BC. Falls among the elderly: A review of the methods and conclusions of epidemiologic studies. J Am Geriatr Soc 1982;30:367-371.

2. Dijcks BPJ, Neyens JCL, Schols JMGA, Haastregt JCM van, Crebolder HFJM, Witte LP de. Valincidenten in verpleeghuizen: gemiddeld bijna 2 per bed per jaar met bij 1,3\% een fractuur als gevolg. Ned Tijdschr Geneeskd 2005;149:1043-7.

3. Rubenstein LZ, Josephson KR, Robbins AS (1994). Falls in the nursing home. Ann Intern Med 1994;121:442-451.

4. Vermeulen HBM (1994). Vallen en valpreventie in het verpleeghuis: een interventiestudie naar het effect van een bed-alarm-systeem. Nijmegen, academisch proefschrift Katholieke Universiteit Nijmegen.

5. Dijk van PTM, Meulenburg OGRM, Sande HJ van de et al. Falls in dementia patients. Gerontologist 1993;33(2):200-204.

6. Graafmans WC, Ooms ME, Hofstee HMA et al. Falls in the elderly: a prospective study of risk factors and risk profiles. Am J Epidemiol 1996;143:1129-36.

7. Buchner DM, Larson EB. Transfer bias and the association of cognitive impairment with falls. J Gen Intern Med 1988;3:254-9.

8. Janti PO, Pyykko VI, Hervonen AL. Falls among elderly nursing home residents. Public Health 1993;107:89-96.

9. Kiely D, Kiel D, Burrows et al. Identifying nursing home residents at risk of falling. J Am Geriatr Soc 1998;46:551-555.

10. Campbell AJ, Borrie MJ, Spears GF et al. Circumstances and consequences of falls experienced by a community population 70 years and over during a prospective study. Age Ageing 1990;19:136-41.

11. Nevitt MC, Cummings SR, Hudes ES. Risk factors for injurious falls: a prospective study. J Gerontol 1991;46:M164-70.

12. Tromp AM, Smit JH, Deeg DJH et al. Predictors for falls and fractures in the longitudinal Aging Study Amsterdam. J Bone Min Res 1998;13:1932-9.

13. Tinetti ME, Speechley M, Ginter SF. Risk factors for falls among elderly persons living in the community. N Engl J Med 1988;319:1701-7.

14. Tinetti M. Factors associated with serious injuries during falls in ambulatory nursing home residents. J Am Geriatr Soc 1987;35:644-648.

15. Keene GS, Parker MJ, Pryor GA. Mortality and morbidity after hip fractures. $\mathrm{Br} \mathrm{Med} \mathrm{J}$ 1993;307:1248-50.

16. Gillespie LD, Gillespie WD, Robertson MC et al. Interventions for preventing falls in elderly people. The Cochrane Library, Issue 2, 2004. 


\section{Chapter 3}

17. American Geriatrics Society, British Geriatrics Society and American Academy of Orthopaedic Surgeons Panel on Fall Prevention. Guideline for the Prevention of Falls in Older Persons. J Am Geriatr Soc 2001;49:664-672.

18. CBO, Kwaliteitsinstituut voor de Gezondheidszorg. Richtlijn Preventie van valincidenten bij ouderen. Alphen aan de Rijn: Van Zuiden Communications BV, 2004.

19. Feder G, Cryer C, Donovan S, Carter Y. Guideline for the prevention of falls in people over 65. BMJ 2000;321:1007-11.

20. Chang JT, Morton SC, Rubenstein LZ et al. Interventions for the prevention of falls in the older adults: systemic review and meta-analysis of randomised clinical trials. BMJ 2004;328:680-686.

21. National Institute for Clinical Excellence. Falls: the assessment and prevention of falls in older people. November 2004.

22. Oakley A, Strange V, Bonell C et al. Process evaluation in randomised controlled trials of complex interventions. BMJ 2006;332:413-6.

23. Rychetnik L, Frommer M, Hawe P et al. Criteria for evaluating evidence on public health interventions. J Epidemiol Community Health 2002;56:119-27.

24. Rubenstein LZ, Robbins AS, Josephson KR et al. The value of assessing falls in an elderly population. A randomised clinical trial. Ann Intern Med 1990;113:308-316.

25. Mulrow CD, Gerety MB, Kanten D et al. A randomised trial of physical rehabilitation for very frail nursing home residents. JAMA 1994 Feb 16;271(7):519-24.

26. Ray WA, Taylor JA, Weador KG et al. A randomized trial of a consultation service to reduce falls in nursing homes. JAMA 1997;278:557-562.

27. Mc Murdo MET, Millar AM, Daly F. A randomized controlled trial of fall prevention strategies in old peoples' homes. Gerontology 2000;46:83-87.

28. Jensen J, Lundin-Olsson L, Nyberg L et al. Fall and injury prevention in older people living in residential care facilities. Ann Intern Med 2002;136:733-741.

29. Jensen J, Nyberg L, Gustafson Y et al. Fall and injury prevention in residential care. Effects in residents with higher and lower levels of cognition. J Am Geriatr Soc 2003; 5:627-635.

30. Jensen J, Nyberg L, Rosendahl E et al. Effects of a fall prevention program including exercise on mobility and falls in frail older people living in residential care facilities. Aging Clin Exp Res 2004;16:283-292.

31. Becker $\mathrm{C}$, Kron M, Lindemann $U$ et al. Effectiveness of a multifaceted intervention on falls in nursing home residents. J Am Geriatr Soc 2003;51:306-313.

32. Bischoff HA, Stahelin HB, Dick $W$ et al. Effects of vitamin D and calcium supplementation on falls: a RCT. J Bone Miner Res 2003;18(2):343-351.

33. Meyer G, Warnke A, Bender R et al. Effect on hip fractures of increased use of hip protectors in nursing homes: cluster randomized controlled trial. BMJ 2003;326:76-8. 
34. Schnelle JF, Kapur K, Alessi C et al. Does an exercise and incontinence intervention save healthcare costs in a nursing home population? J Am Geriatr Soc 2003;51:161-168.

35. Schoor NM van, Smit H, Twisk J et al. Prevention of hip fractures by external hip protectors. JAMA 2003;289:1957-1962.

36. Dyer CAE, Taylor GJ, Reed $M$ et al. Falls prevention in residential care homes: a randomised controlled trial. Age and Ageing 2004;33:596-602.

37. Kerse N, Butler M, Robinson E et al. Fall prevention in residential care: a cluster, randomised, controlled trial. J Am Geriatr Soc 2004;52:524-531.

38. Ray WA, Taylor JA, Brown AK et al. Prevention of fall-related injuries in long-term care. A randomized controlled trial of staff education. Arch Intern Med. 2005;165:2293-98.

39. Schnelle JF, Alessi CA, Simmons SF et al. Translating clinical research into practice: a randomized controlled trial of exercise and incontinence care with nursing home residents. J Am Geriatr Soc 2002;50:1476-83. 



\title{
Chapter 4
}

The development of a multidisciplinary fall risk evaluation tool for demented nursing home patients in the Netherlands

\author{
J.C.L. Neyens, B.P.J. Dijcks, J.C.M. van Haastregt, L.P. de Witte,
} W.J.A. van den Heuvel, H.F.J.M. Crebolder, J.M.G.A. Schols.

BMC Public Health 2006; 6: 74. 


\section{ABSTRACT}

\section{Background}

Demented nursing home patients are at high risk for falls. Falls and associated injuries can have a considerable influence on the autonomy and quality of life of patients. The prevention of falls among demented patients is therefore an important issue. In order to intervene in an efficient way in this group of patients, it is important to systematically evaluate the fall risk profile of each individual patient so that for each patient tailor-made preventive measures can be taken. Therefore, the objective of the present study is to develop a feasible and evidence based multidisciplinary fall risk evaluation tool to be used for tailoring preventive interventions to the needs of individual demented patients.

\section{Methods}

To develop this multidisciplinary fall risk evaluation tool we have chosen to combine scientific evidence on the one hand and experts' opinions on the other hand. Firstly, relevant risk factors for falling in elderly persons were gathered from the literature. Secondly, a group of Dutch experts in the field of falls and fall prevention in the elderly were consulted to judge the suitability of these risk factors for use in a multidisciplinary fall risk evaluation tool for demented nursing home patients. Thirdly, in order to generate a compact list of the most relevant risk factors for falling in demented elderly, all risk factors had to fulfil a set of criteria indicating their relevance for this specific target population. Lastly the final list of risk factors resulting from the above mentioned procedure was presented to the expert group. The members were also asked to give their opinion about the practical use of the tool.

\section{Results}

The multidisciplinary fall risk evaluation tool we developed includes the following items: previous falls, use of medication, locomotor functions, and (correct) choice and use of assistive and protective devices. The tool is developed for the multidisciplinary teams of the nursing homes.

\section{Conclusions}

This evidence and practice based multidisciplinary fall risk evaluation tool targets the preventive interventions aimed to prevent falls and their negative consequences in demented nursing home patients. 


\section{BACKGROUND}

Falls and fall-related injuries are a major problem in community residing elderly persons and even more in frail elderly residing in institutions. Fall incidents occur frequently in nursing homes and may have considerable consequences for the health status and quality of life of the patients involved, especially if the fall results in a hip fracture. In the Netherlands the mean incidence of fractures for psychogeriatric patients in nursing homes is 26.3 to 28.8 per 1000 beds per year. ${ }^{1}$ Due to these fractures, not only the nursing care load increases, but also the mortality risk of patients.

Dementia is a major risk factor for falling. ${ }^{2-4}$ Demented patients show a gradually deteriorating mobility and a diminishing ability to recognise, judge and avoid hazards. In Dutch nursing homes about $55 \%$ of the patients suffer from dementia but they are involved in $75 \%$ of the fall incidents. ${ }^{5}$ Therefore, it can be concluded that all demented patients in nursing homes are at high risk of falls. This stresses the importance of taking adequate preventive measures to prevent falls in this group of patients.

Research data indicate positive effects of multifactorial interventions targeted at the prevention of fall incidents. ${ }^{6}$ This evidence mostly concerns community dwelling people. Despite the magnitude of the problem of fall incidents in (demented) nursing home patients, only limited evidence is available for the effectiveness of fall prevention among these patients. ${ }^{6,11}$ Fall risk assessment tools and preventive interventions developed for the general population of elderly persons seem to be inappropriate for demented patients.

The present study aims to contribute to the development of a specialised fall prevention intervention for demented nursing home patients, feasible for the nursing home staff. In order to intervene in an efficient way in the group of demented patients (who all can be considered to be at high risk for falls), it is important to systematically evaluate the fall risk profile of each individual patient so that for each patient tailor-made preventive measures can be taken. Therefore, the objective of the study presented in this article is to develop a feasible and evidence based multidisciplinary fall risk evaluation tool to be used for tailoring preventive interventions to the needs of individual demented patients.

\section{Methods}

The development of this multidisciplinary fall risk evaluation tool consisted of the following four steps: 
1. Searching the literature for risk factors for falling;

2. First consultation of experts: suitability of factors;

3. Final selection of risk factors;

4. Second consultation of experts: practical use of the tool.

Below the methods used in each step are described.

\section{Step 1: Searching the literature for risk factors for falling}

A search in PubMed, Medline and Cinahl (from January 1986 until July 2002) was performed to collect scientific publications about risk factors for falling. The search strategy used was: [fall(s) AND elderly] AND [nursing home(s) OR long term care OR risk factor(s) OR assessment OR dementia]. The abstracts of the publications found were screened in order to make a first selection of potentially relevant papers. All papers that addressed risk factors for falling in the elderly were included in this first selection, irrelevant of whether they referred to elderly people residing in the community, hospitals or institutions for long term care. The full text of the publications included, were retrieved and the papers were screened for relevant information about risk factors for falling among elderly people (65+). Subsequently a list of risk factors for falls was made. A risk factor was included in the list if a relationship between the factor and falls in the elderly was reported.

\section{Step 2: First consultation of experts: suitability of factors}

A group of national experts $(n=11)$ in the field of falling, fall prevention, guideline development and implementation was assembled. The members of this group were researchers from the Free University Amsterdam (VU), Maastricht University (UM), the Dutch Organisation for Applied Scientific Research (TNO), representatives of different disciplines working in a nursing home (nursing home physician, nurse, physiotherapist and occupational therapist), a representative of the Dutch Branch Organisation for Nursing Homes (Arcares), and a representative of the Dutch Association of Nursing Home Physicians (NVVA).

In a plenary meeting the large list of risk factors resulting from step 1 was presented to the experts. Each expert was asked to judge for each risk factor whether it seemed relevant to include it in a multidisciplinary fall risk evaluation tool for demented nursing home patients. They were asked to take into account the daily care process in Dutch nursing homes. In the Netherlands it is common to perform a general comprehensive assessment shortly after 
admission of a patient to the nursing home. The multidisciplinary fall risk evaluation tool should not overlap with this assessment but should be complementary to it.

In order to generate a compact list of the most relevant risk factors for demented nursing home patients, we reduced the list of risk factors resulting from step 1 using the following criterion: during the expert meeting at least $75 \%$ of the experts present had to agree on the importance of this factor.

\section{Step 3: Final selection of risk factors}

Subsequently the members of the research team wanted to make a further selection of the factors resulting from step 2, in order to compose a feasible multidisciplinary fall risk evaluation tool. To do this, they developed the following list of criteria:

- the factor has been described as a risk factor for falling in at least one article addressing nursing home care;

- evaluation of the factor among demented nursing home patients has to be possible;

- the factor must be modifiable; and

- appropriate interventions to reduce or eliminate the risk factor among demented nursing home patients (applicable in daily nursing home routine) are or can be made available.

Next the research team assessed whether the factors resulting from the first selection matched these criteria. Because this did not lead to a substantial reduction of the number of factors, the following criterion was added to further reduce the number of factors:

- the reported Odds Ratio/Relative Risk Ratio of the factor has to be 1.5 or higher.

\section{Step 4: Second consultation of experts: practical use of the tool}

The list of factors resulting from step 3 was presented to the participating experts. By means of a structured (e-mail) questionnaire, we asked them to give their opinion regarding aspects of the practical use of the multidisciplinary fall risk evaluation tool. For every risk factor incorporated in the tool, the experts had to judge (yes or no) the proposals of the research team regarding how, by whom and at which moment in the care process it should be assessed. If the majority answered positive the tool became final. 
Table 2. Risk factors for falls, related to the inclusion criteria

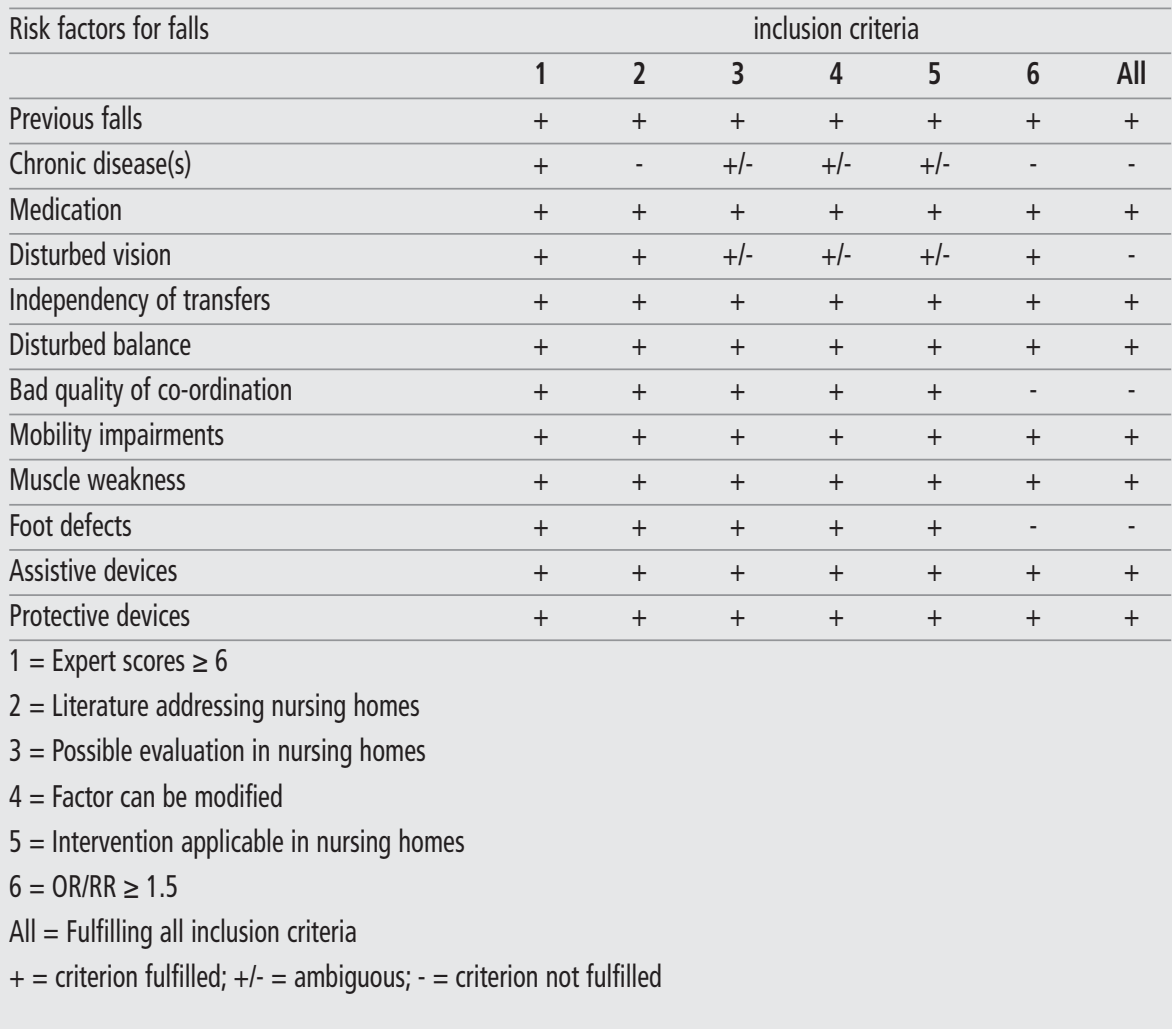

multidisciplinary fall risk evaluation tool among demented nursing home patients, according to at least 6 of the 7 experts present were: previous falls, chronic disease(s), medication, disturbed vision, independency of transfers, disturbed balance, bad quality of co-ordination, mobility impairments, muscle weakness, foot defects, assistive devices, and protective devices.

\section{Final selection of risk factors}

Table 2 shows the results of the final selection procedure of items for the multidisciplinary fall risk evaluation tool. The risk factors that are shown in this table are those that are considered to be relevant by 6 out of 7 experts. Column 1 shows the required expert group consensus score. Column 2 shows if the items in question are mentioned in literature addressing nursing homes. Columns 3 
and 4 show respectively the possibility for evaluation in daily nursing home practice and whether the risk factors can be modified. Column 5 shows whether interventions to reduce or eliminate the risk factors (applicable in nursing homes), are or can be made available. Column 6 shows the scores on the additional criterion $(O R / R R \geq 1.5)$ to further reduce the list. Column 7 shows which factors fulfilled all inclusion criteria. Eight factors fulfilled all criteria. The final multidisciplinary fall risk evaluation tool therefore includes:

- previous falls; a positive fall history in the preceding 6 months predicts future falls ${ }^{13-17,19,26-29,33-35,38,44,51}$

- medication; number, type and doses of drugs as well as times of intake can influence the risk of falling

- locomotor functions; the factors muscle weakness, mobility impairments, disturbed balance, and independency of transfers, which all can increase the risk of falling, were taken together as one item called locomotor functions

- assistive and protective devices; for the assistive and protective devices, both the choice and the use of them have to be correct in order to create safe conditions for the patient because wrong choice and/or use enhances the fall risk.

\section{Second consultation of experts: practical use of the tool}

Eight experts responded to the (e-mail) questionnaire. A majority agreed on involving different disciplines in the fall risk evaluation tool, stressing the multidisciplinary aspects of the tool and the importance incorporating the tool in a cyclic procedure: fall risk evaluation at admission; an evaluation after a fall accident; an evaluation at request of the ward; and a periodical repetition of the tool two times a year. Based on the answers of the experts, the members of the research team developed practical guidelines regarding the use of the multidisciplinary fall risk evaluation tool on the psychogeriatric wards. These guidelines are presented in table 3, and described in more detail below.

\section{Previous falls}

A fall is defined as an event which results in a person coming to rest advertently on the ground or other level (adjusted version of the definition of the Kellogg International Work Group). ${ }^{52}$ At admission to the nursing home, information with regard to the fall history in the previous 6 months has to be gathered from the general practitioner, family members and if possible from the patients themselves. Because information about the fall history is gathered retrospectively it is important 
to use more than one source of information, whenever possible. Obviously the self-report of falls among demented persons may be very unreliable due to their cognitive problems.

The information that has to be gathered includes the number of fall accidents, the possible causes and circumstances of the fall, the consequences of the fall, and the preventive actions already taken. ${ }^{53}$ Although obviously fall history it self cannot be influenced, the analysis of the fall history allows the nursing home team to assess whether the factors which caused the falls in this specific patient can be influenced. The more we know about the fall history, the better we can anticipate upon the fall related causes and circumstances. The fall history allows the team also to evaluate the fall preventive policy with regard to individual patients.

\section{Medication}

The medication used by the patient has to be registered by the nursing home physician who will consequently assess its influence on fall risk. The number of drugs as well as the type, doses and time of intake must be registered. The combination of four or more drugs enhances the risk of falling. Some drugs, particularly cardiovascular and psychotropic drugs, the latter often used in dementia patients, are known to have possible side effects such as drowsiness, dizziness, unstable blood pressure and confusion and thereby can enhance fall risk. ${ }^{13,15-17,19,21,23,26-29,32,34,38,44,45,51}$

Regular monitoring of the resident's medication is important because in most nursing home patients, including the demented, we observe polypharmacy, with all possible negative implications.

\section{Locomotor functions}

Clinical judgement of the patients' mobility by the multidisciplinary team occurs in every day routine and gives important information about possible problems with standing, walking, activities of daily living and transfers and with regard to wandering behaviour, restlessness during the night and other risky behaviour. ${ }^{2,6,13-29,31-33,35-39,41,44,50,51}$ In addition to this clinical judgment the Barthel score and the Tinetti test (POMA) are valid screening tools, enabling us to measure more specifically the activities of daily living and the functioning of the mobility apparatus. ${ }^{30,55}$ The Tinetti test assesses both the balance (9 items) and the gait ( 6 items). The maximum score is 28 points. The scores 19-24 indicate an increased risk of falling. The scores below 19 indicate great risk of falling. The Tinetti test has important practical use: the scores tell us either to focus on balance, on gait or on both. Nurses can observe the general, functional 


\section{Chapter 4}

locomotor functions during daily activities; an occupational therapist or a physiotherapist can perform the more specific tests.

\section{Assistive and protective devices}

Taking into account the physical abilities and mental status of the patients involved, the evaluation of both correct choice and use of assistive (walking aids, transfer aids) and protective (external hip protector, alarm cushions/ sensors) devices, can be performed by the clinical judgment of nurses, physiotherapists and occupational therapists. ${ }^{2,14,30,31,47}$

\section{Tailoring interventions based on the fall risk evaluation}

Based on the results of the fall risk evaluation the nursing home staff using the tool has to decide what specific fall preventive interventions are needed for each individual patient. These interventions could include:

- anticipating upon the causes and circumstances of the fall;

- critically reviewing and monitoring medication intake (type, number, dose and time of intake);

- offering exercise programmes specifically targeted at the needs of the individual patient;

- carefully reassessing the need for assistive and protective devices, and promoting the correct use of these devices.

Table 3. Practical use of the multidisciplinary fall risk evaluation tool for demented nursing home patients

\begin{tabular}{|c|c|c|c|}
\hline What? & How? & Who? & When? \\
\hline \multirow[t]{3}{*}{ Previous falls } & data from GP & nursing home physician & \\
\hline & (hetero) anamnesis & nurses/carers & \\
\hline & fall registration ${ }^{52}$ & & At admission \\
\hline Medication & medication list & nursing home physician & After a fall incident \\
\hline \multirow[t]{2}{*}{ Locomotor functions } & Barthel index ${ }^{30}$ & nurses/carers & At request ward \\
\hline & Tinetti (POMA) $)^{55}$ & $\begin{array}{l}\text { occupational therapist } \\
\text { physiotherapist }\end{array}$ & $\begin{array}{l}\text { Periodical repetition } \\
\text { ( } 2 \text { x per year) }\end{array}$ \\
\hline \multirow{3}{*}{$\begin{array}{l}\text { Assistive and } \\
\text { protective devices }\end{array}$} & Check-list concerning & nurses/carers & \\
\hline & - choice & occupational therapist & \\
\hline & - use & physiotherapist & \\
\hline
\end{tabular}




\section{Discussion}

The aim of this study was to develop a feasible and evidence based multidisciplinary fall risk assessment tool for multidisciplinary teams in wards for demented nursing home patients. The tool evaluates five important fall risk factors in demented elderly: previous falls, use of medication, locomotor functions, and (correct) choice and use of assistive and protective devices. The disciplines that may be involved in using this tool are nursing home physicians, nurses, occupational therapists and physiotherapists. It is important to incorporate the multidisciplinary fall risk assessment tool in a cyclic monitoring procedure. The results of executing this tool target the multidisciplinary and multifactorial fall prevention activities tailored for each individual patient.

\section{Methodological considerations}

A strong aspect of this multidisciplinary fall risk assessment tool is that it is both evidence and practice based. This has resulted in a tool, which is supported by evidence in scientific literature, and also seems to fit easily into the daily nursing home routine. The fact that we partly based the tool on opinions of experts, however, may at the same time be considered as a weak point of the study because the results obviously depend on the specific composition of the expert group. Despite that, in our opinion the group contributing to the development of the tool was well balanced and representative for the available Dutch expertise on falls in the community as well as in institutions for chronic care.

Obviously, performing this multidisciplinary fall risk assessment tool in demented nursing home patients does not guarantee that all risk factors for falls in an individual patient will be detected. Therefore it remains very important that sufficient attention is paid to interventions with regard to other factors that in the opinion of nursing home physicians, nurses, and/or paramedical staff can contribute to falls in individual patients.

\section{Practical implications}

At this moment most nursing homes in the Netherlands do not have specific guidelines or structured programmes for the prevention of fall incidents among demented patients. ${ }^{1}$ In developing such guidelines the multidisciplinary fall risk evaluation tool, as presented here, involves the first step of an effective fall prevention programme. In addition, complete and integral fall prevention programmes should also include possible interventions that may be targeted by the results of using this evaluation tool. Of course, these programmes also 


\section{Chapter 4}

should provide information about general fall prevention activities, for instance, educational programs for the nursing home team, and realisation of a safe nursing home environment. ${ }^{5,57}$

Currently we are performing a randomised controlled trial among demented nursing home patients in the Netherlands in which we are testing the effectiveness of the fall risk evaluation tool and the multifactorial interventions, specifically targeted by this tool. ${ }^{58}$ The trial is accompanied by an evaluation of the feasibility and acceptability of the fall risk evaluation tool, in order to further optimise it and to make it more suitable for use in daily practice.

\section{Conclusions}

This evidence and practice based multidisciplinary fall risk assessment tool can form the basis for a multifactorial and multidisciplinary intervention aimed to prevent falls and their negative consequences in demented nursing home patients. 


\section{References}

1. Dijcks BPJ, Neyens JCL, Schols JMGA, van Haastregt JCM, Crebolder HFJM, de Witte LP. Valincidenten in verpleeghuizen: gemiddeld bijna 2 per bed per jaar met bij 1,3\% een fractuur als gevolg. Ned Tijdschr Geneeskd 2005, 149: 1043-7

2. Rubenstein LZ, Josephson KR, Robbins AS (1994). Falls in the nursing home. Ann Intern Med 1994, 121: 442-451

3. Vermeulen HBM (1994). Vallen en valpreventie in het verpleeghuis: een interventiestudie naar het effect van een bed-alarm-systeem. Nijmegen, academisch proefschrift Katholieke Universiteit Nijmegen.

4. Dijk van PTM, Meulenburg OGRM, Sande HJ van de, Habbema JDF. Falls in dementia patients. Gerontologist 1993, 33(2): 200-204

5. Schols JMGA, Crebolder HFJM, Weel C van. Nursing home and nursing home physician: the Dutch experience. J. Am Med Dir Assoc 2004, 5: 207-212

6. Gillespie LD, Gillespie WJ, Robertson MC, Lamb SE, Cumming RG, Rowe BH. Interventions for Preventing Falls in Elderly People. The Cochrane Library 2002, issue 2.

7. American Geriatrics Society, British Geriatrics Society and American Academy of Orthopaedic Surgeons Panel on Fall Prevention. Guideline for the Prevention of Falls in Older Persons. JAGS 2001, 49: 664-672

8. Perell KL, Nelson A, Goldman RL, Luther SL, Prieto-Lewis N, Rubenstein LZ. Fall risk assessment measures: an analytic review. Journal of Gerontology: Medical Sciences 2000, 56: M761-M766

9. Boers I, Willibald G, Stalenhoef PA, Bloem BR (2001). Falls in the elderly. Wien Klin Wochenschr 2001, 113/11-12: 398-407

10. Moreland. A meta-analysis of fall prevention programs for the elderly: how effective are they? Nursing Research 2002, 51(1): 1-8

11. Ray WA, Taylor JA, Meador KG, Thapa PB, Brown AK, Kajihara HK et al. A randomized trial of a consultation service to reduce falls in nursing homes. JAMA 1997, 278: 557-562

12. Ooi WL, Hossain M, Lipsits LA (2000). The assocation between orthostatic hypotension and recurrent falls in nursing home residents. Am J Med 2000, 108: 106-111

13. Bueno-Cavanillas A, Padilla-Ruiz F, Jiménez-Moleón JJ, Peinado-Alonso CA, GálvezVargas R. Risk factors in falls among the elderly according to extrinsic and intrinsic precipitating causes. Eur J Epidemiol 2000, 16: 849-859

14. Kiely DK, Kiel DP, Burrows AB, Lipsitz LA. Identifying nursing home residents at risk for falling. JAGS 1998, 46: 551-555

15. Krueger PD, Brazil K, Lohfeld LH. Risk factors for falls and injuries in a long-term care facility in Ontario. Can J Public Health 2001, 92: 117-120 


\section{Chapter 4}

16. Myers AH, Baker SP, Van Natta ML, Abbey H, Robinson EG. Risk factors associated with falls and injuries among elderly institutionalized persons. Am J Epidemiol 1991, 133: $1179-1190$

17. Thapa PB, Gideon P, Brockman KG, Fought RL, Ray WA. Clinical and biomechanical measures of balance as fall predictors in ambulatory nursing home residents. I Gerontol Med Sci 1996, 51A: M239-M246

18. Thapa PB, Brockman KG, Gideon P, Fought RL, Ray WA. Injurious falls in nonambulatory nursing home residents: a comparative study of circumstances, incidence and risk factors. JAGS 1996, 44: 273-278

19. Graafmans WC, Ooms ME, Hofstee HMA, Bezemer PD, Bouter LM, Lips P. Falls in the elderly: a prospective study of risk factors and risk profiles. Am J Epidemiol 1996, 143(11): 1129-1136

20. Luukinen H, Koski K, Laippala P, S-L Kivela. Risk factors for recurrent falls in the elderly in long-term institutional care. Pub Health 1995, 109: 57-65

21. Lipsitz LA, Jonsson PV, Kelley MM, Koestner JS. Causes and correlates of recurrent falls in ambulatory frail elderly. J Gerontol Med Sci 1991, 46: M114-M122

22. Maki BE. Gait changes in older adults: predictors of falls or indicators of fear? JAGS 1997, 45: 313-320

23. Yip YB, Cumming RG. The association between medications and falls in Australian nursing-home residents. Med J Aust 1994, 160: 14-18

24. Rawsky E. Review of the literature on falls among the elderly. Image J Nurs Sch 1998, 30: 47-52

25. Robbins AS, Rubenstein LZ, Josephson KR, Schulman BL, Osterweil D, Fine G. Predictiors of falls among elderly people: results of two population-based studies. Arch Intern Med 1989, 149: 1628-1633

26. Tinetti ME, Speechley M, Ginter SF. Risk factors for falls among elderly persons living in the community. N Engl / Med 1988, 319: 1701-1707

27. Bueno Cavanillas A, Padilla Ruiz F, Peinado Alonso C, Espigares Garcia M, Galvez Vargas R. Factores de riesgo de caidas en una poblacion anciana institucionalizada. Estudio de cohorts prospectivo. (Risk factors associated with falls in institutionalized elderly persons. Prospective cohort study. Med Clin (Barc) 1999, 112: 10-15

28. Nurmi I Sihvonen M, Kataja M, Lüthje P. Falls among institutionalized Elderly a prospective study in four institutions in finland. Scand J Caring Sci 1996, 10: 212-220

29. American Medical Directors Association (AMDA) and the American Health Care Association. Falls and Fall Risk. 1997.

30. Mahoney FI, Barthel D. Functional evaluation: the Barthel Index. Md Med J 1965, 14: 61-65

31. Graafmans WC, Lips P, Wijlhhuizen GJ, Pluijm SM, Bouter LM. Daily physical activity and the use of a walking aid in relation to falls in elderly people in a residential care setting. Z Gerontol Geriatr 2003, 36: 23-28 
32. Campbell AJ, Borrie MJ, Spears GF. Risk factors for falls in a community-based prospective study of people 70 years and older. J Gerontol 1989, 44: M112-7

33. Stalenhoef PA, Diederiks JPM, Knottnerus JA, Kester ADM, Crebolder HFJM. A risk model for the prediction of recurrent falls in the community-dwelling elderly: a prospective cohort study. J Clin Epidemiol 2002, 55: 1088-94

34. Luukinen H, Koski K, Kivela S-L, Laippala P. Social status, life changes, housing conditions, health, functional abilities and life-style as risk factors for recurrent falls among the home-dwelling elderly. Public Health 1996, 110: 115-8

35. Tinetti ME, Speechley M, Ginter SF. Risk factors for falls among elderly persons living in the community. N Engl J Med 1988, 319: 1701-7

36. Davis WJ, Ross PD, Nevitt MC, Wasnich RD. Risk factors for falls and for serious injuries on falling among older Japanese women in Hawaii. J Am Geriatr Soc 1995, 43: 1214-21

37. O'Loughlin JL, Robitaille Y, Boivin JF, Suissa S. Incidence of and risk factors for falls and injurious falls among the community-dwelling elderly. Am J Epidemiol 1993, 137: 342-54

38. Tromp AM, Pluijm SMF, Smit HJ, Deeg DJH, Bouter LM, Lips P. Fall-risk screening test: a prospective study on predictors for falls in community-dwelling elderly. J Clin Epidemiol 2001, 54: 837-44

39. Lord SR, Dayhew J. Visual risk factors for falls in older people. J Am Geriatr Soc 2001, 49: 508-15

40. Schoor NM van, Smit JH, Pluijm SMF, Jonker C, Lips P. Different cognitive functions in relation to falls among older persons. Immediate memory as an independent risk factor for falls. J Clin Epidemiol 2002, 55: 855-62

41. Tinetti ME, Inouye SK, Gill TM, Doucette JT. Shared risk factors for falls, incontinence and functional dependence. Unifying the approach to geriatric syndromes. JAMA 1995, 273: $1348-53$

42. Oliver D, Britton M, Seed P, Martin FC, Hopper AH. Development and evaluation of evidence based risk assessment tool (STRATIFY) to predict which elderly inpatients will fall: case-control and cohort studies. BMJ 1997, 315: 1049-53

43. Tinetti ME, Williams TF, Mayewski R (1986). Fall risk index for elderly patient based on number of chronic disabilities. Am J med 1986, 80: 429-434

44. Vasallo M, Sharma JC, Allen SC. Characteristics of single fallers and recurrent fallers among hospital in-patients. Gerontology 2002, 48: 147-50

45. Tituarima JA, Meulen JH van der, Haan RJ de, Straten A van, Limburg M. Risk factors for falls of hospitalized stroke patients. Stroke 1997, 28: 297-301

46. Tideiksaar R. Falls in old age: its prevention and treatment. New York: Springer 1989.

47. Neufeld RR, Libow LS, Foley WJ, Dunbar JM, Cochen C, Breuer B. Restraint reduction reduces serious injuries among nursing home residents. J Am Geriatr Soc.1999, 47: $1202-1207$ 


\section{Chapter 4}

48. Close J, Ellis M, Hooper R, Glucksman E, Jackson S, Swift C. Prevention of falls in the elderly trial (PROFET): a randomised controlled trial. Lancet 1999, 353: 93-7

49. Haastregt JC van, Diederiks JP, Rossum E van, Witte LP de, Voorhoeve PM, Crebolder HF. Effects of a programme of multifactorial home visits on falls and impairments in elderly people at risk: a randomised controlled trial. BMJ 2000, 321: 994-8

50. Feder G, Cryer C, Donovan S, Carter Y. Guideline for the prevention of falls in people over 65. BMJ 2000, 321: 1007-11

51. Stalenhoef PA, Crebolder HFJM, Knottnerus JA, Horst FGEM. Incidence, risk factors and consequences of falls among elderly subjects living in the community: a criteria based analysis. Eur J Pub Health 1997, 7: 328-34

52. Arcares. Melding Incidenten Cliënten in Verpleeg- en Verzorgingshuizen. Arcares rapport oktober 2002.

53. Kellogg International Work Group on the prevention of falls by the elderly.

54. Woollacot MH, Shumway-Cook A. Concepts and methods for assessing postural instability. J Aging Phys Activ 1996, 4: 214-233

55. Tinetti ME. Performance-oriented assessment of mobility problems in elderly patients. JAGS 1986, 34: 119-126

56. Kinkelder A de, Dierkx RIJ. Functionele mobiliteitstests voor het valrisico bij verpleeghuispatiënten, een literatuur onderzoek naar de diagnostische waarde. Tijdschr Gerontol Geriatr 2001, 32: 69-73

57. Rubenstein LZ, Robbins AS, Josephson KR, Schulman BL, Osterweil D. The value of assessing falls in an elderly population. A randomized clinical trial. Annals of Internal Medicine 1990, 113: 308-316

58. Neyens JCL, Dijcks BPJ, Schols JMGA, Haastregt JCM van, Heuvel WJA van den, Crebolder HFJM, Witte LP de. Ontwikkeling en implementatie van een multifactoriële interventie gericht op de preventie van valincidenten en de gevolgen daarvan bij psychogeriatrische verpleeghuispatiënten. TVV 2002, 26: 24-28 



\section{Chapter 5}

\section{A multifactorial intervention}

for the prevention of falls in psychogeriatric nursing home patients: randomised controlled trial

J.C.L. Neyens, B.P.J. Dijcks, J.W. Twisk, J.M.G.A. Schols, J.C.M. van Haastregt, W.J.A. van den Heuvel, L.P. de Witte. 


\section{Chapter 5}

\section{Abstract}

\section{Objective}

To evaluate the effectiveness of a multifactorial intervention on incidence of falls and injuries falls in psychogeriatric nursing home patients.

\section{Design}

Cluster-randomised controlled 12-month trial.

\section{Setting}

Psychogeriatric wards in 12 nursing homes in The Netherlands.

\section{Participants}

Psychogeriatric nursing home patients $(n=518)$.

\section{Intervention}

A general medical assessment and an additional specific fall risk evaluation tool, applied by a multidisciplinary fall prevention team, resulting in general and individual fall prevention activities.

\section{Measurements}

Falls and injurious falls.

\section{Results}

There were 355 falls in 169.5 patient years $(=2.09$ falls per patient per year) in the intervention group and 422 falls in 166.3 patient years $(=2.54$ falls per patient per year) in the control group. The number of injurious falls was 0.69 per patient per year in the intervention group and 0.67 in the control group. Intention-to-treat analysis, with adjustment for ward-related and patient-related parameters, and intra-cluster correlation, showed that the intervention group had a significantly lower mean fall incidence rate than the control group (rate ratio $=0.64,95 \% \mathrm{Cl}=0.43-0.96, \mathrm{p}=0.029)$. Subgroup analyses showed that fall risk declined further as patients participated longer in the intervention programme. Furthermore, the intention-to-treat analysis also showed a lower mean incidence rate for injurious falls in the intervention group, but this difference did not reach statistical significance (rate ratio $0.54,95 \% \mathrm{Cl}=0.29$ 1.01, $p=0.054)$. 


\section{Conclusion}

The introduction of a structured multifactorial intervention to prevent falls in psychogeriatric nursing home patients significantly reduces the number of falls. This reduction is substantial and of high clinical relevance. Significant effect on injurious falls could not be demonstrated.

\section{Key Words}

accidental falls; prevention; older people; long-term care; dementia 


\section{INTRODUCTION}

Falls and fall-related injuries are a major problem in community residing elderly persons and even more in frail elderly residing in institutions. ${ }^{1,2}$ Falls are a frequent occurrence in nursing homes, with approximately two falls per bed per year. ${ }^{3}$ Falls may have considerable consequences for the health status, the autonomy and the quality of life of the patients involved. Falls in cognitively impaired elderly are of particular concern. ${ }^{4}$ Psychogeriatric nursing home patients especially have a high risk of falling. ${ }^{3-9}$ As the mobility of these patients gradually deteriorates, so does their ability to recognise, judge and avoid hazards. This emphasises the importance of adequate measures to prevent falls in this group of patients.

Research indicates that multifactorial interventions to prevent fall incidents can have positive effects, but most existing evidence concerns community dwelling elderly. ${ }^{10-13}$ There are only a handful of trials concerning residential care facilities, of which some demonstrated that intervention programmes did help to prevent falls. ${ }^{10}$ Only limited evidence is available for the effectiveness of fall prevention among psychogeriatric nursing home patients specifically. ${ }^{14}$ It is therefore important to investigate whether interventions, directed at risk factors, and fall prevention strategies result in a reduction of falls and fall related injuries among psychogeriatric patients.

We developed a multidisciplinary and multifactorial fall prevention intervention for psychogeriatric nursing home patients, based on a review of the literature and consultations with experts on fall prevention and geriatric nursing. ${ }^{15,16}$ The primary objective of the present study was to assess the effectiveness of this intervention on the incidence of falls and additionally on fall related injuries in psychogeriatric nursing home patients in the Netherlands.

\section{Methods}

\section{Design}

The design was a cluster randomised controlled trial (RCT), performed in twelve nursing homes in the Netherlands. Almost all Dutch nursing homes have somatic wards and psychogeriatric wards. Residents with somatic problems (e.g. post stroke status, neurodegenerative diseases etc.) are admitted to somatic wards, which look much alike the wards in long-term care hospitals in the UK. Psychogeriatric wards of Dutch nursing homes are adapted for residents with loss of orientation because of dementia. We may refer here to the specialized 
Alzheimer units in the USA. On a psychogeriatric ward $24 \mathrm{hr}$ multidisciplinary treatment, support and nursing care is provided for demented residents. ${ }^{17}$ Six nursing homes were allocated to the intervention group and six to the control group. Each nursing home selected one psychogeriatric ward. After approval by a certified ethics committee, the RCT started in November 2003. The inclusion period for each ward was twelve months.

In the intervention group, general staff at the nursing homes and professionals on the intervention wards received written and oral information and instructions about the intervention programme and data collection.

In the control group, general staff and managers on the control wards received written and oral instructions about data collection. All patients received usual care.

On the basis of earlier research among Dutch nursing home patients, we estimated the fall rate in our study population at 3.3 per patient per year, with a standard deviation of $2.5 .^{5} \mathrm{~A}$ sample size of 180 patients per group would allow detection of a $30 \%$ reduction in fall rate, with a power of .80 and $5 \%$ significance. On the basis of this power calculation, the minimum sample size for the trial was set at 180 patients per group.

\section{Participants and setting}

Study participants were psychogeriatric patients in twelve Dutch nursing homes of which ten had both somatic wards and psychogeriatric wards, the other two had only psychogeriatric wards. All patients residing on the selected psychogeriatric wards, one per participating nursing home, were included in the study, regardless of whether their stay lasted the entire inclusion period or only part of this period. Of the included patients in the intervention group, only those whose relatives or legal guardians had given us their written informed consent actually participated in the intervention programme.

\section{Randomization}

In 2002 we sent a questionnaire to all 371 nursing homes in the Netherlands, to determine how many falls and fall-related fractures annually occur in these homes and to assess the use of fall prevention protocols. ${ }^{3}$ General staff were asked whether they would agree to participate in our intervention study. Out of 202 responding nursing homes, 119 provisionally agreed to inclusion and met the trial inclusion criteria (a psychogeriatric ward with at least thirty beds and not using a fall prevention protocol). These 119 nursing homes were contacted again in 2003. We gave them more information about the study and the intervention protocol and asked again whether they would participate in the 


\section{Chapter 5}

study. Forty homes did not respond, 45 declined participation and 34 agreed to participate. The last were divided into three groups based on mean fall incidence rate per psychogeriatric bed (low <1.86, medium 1.87-2.61, high $>2.62$ ) in the years 2000 and 2001, as reported in the questionnaire. At random, using computer techniques, two intervention homes and two control homes were selected from each group, resulting in a total of six intervention homes and six control homes. At each home, the management selected the ward to be included in the study, based on the following criteria: at least 30 beds, not using a fall prevention protocol and with the largest number of mobile patients. Three nursing homes had no ward with thirty beds or more so that smaller wards were included instead. In total, the six intervention wards had 177 beds and the six control wards had 171 beds.

\section{Study outcomes}

The primary outcome parameter in the study was the number of falls on the ward during the inclusion period of twelve months. The secondary outcome was the number of injurious falls, categorised as 'fractures' or 'other'.

\section{Intervention programme}

The intervention programme consisted of a general medical assessment focusing on fall risks and a specific fall risk evaluation tool assessing fall history, medication intake, mobility, and the use of assistive and protective aids. ${ }^{16}$ The total risk assessment resulted in general fall prevention activities and individually tailored fall prevention interventions for each patient, e.g. a lower total number of medications used by the patient, an individual tailored exercise programme on balance and an individual tailored walking aid. ${ }^{16}$

Each intervention ward installed a multidisciplinary fall prevention team, consisting of routine staff: a nursing home physician, two nurses, a physiotherapist and an occupational therapist. These teams coordinated the intervention programme during fortnightly fall prevention conferences. They discussed each patient at admission, after a fall, and at the request of professionals on the ward; in any case, at least twice a year, even if there had been no fall incident or request.

General medical assessments were performed by medical staff when a patient was admitted or when there was a change in medical condition. The fall prevention teams carried out the fall risk evaluation of each patient. They discussed the outcome in conjunction with the findings of the general medical assessment and decided which individual fall prevention activities were necessary.

Team members or their colleagues then executed these specific fall prevention activities, which could include any or all of the following: anticipating 
Figure 1. Outline of the intervention programme

General Medical Assessment

- At start trial

- At admission

- Change in medical condition

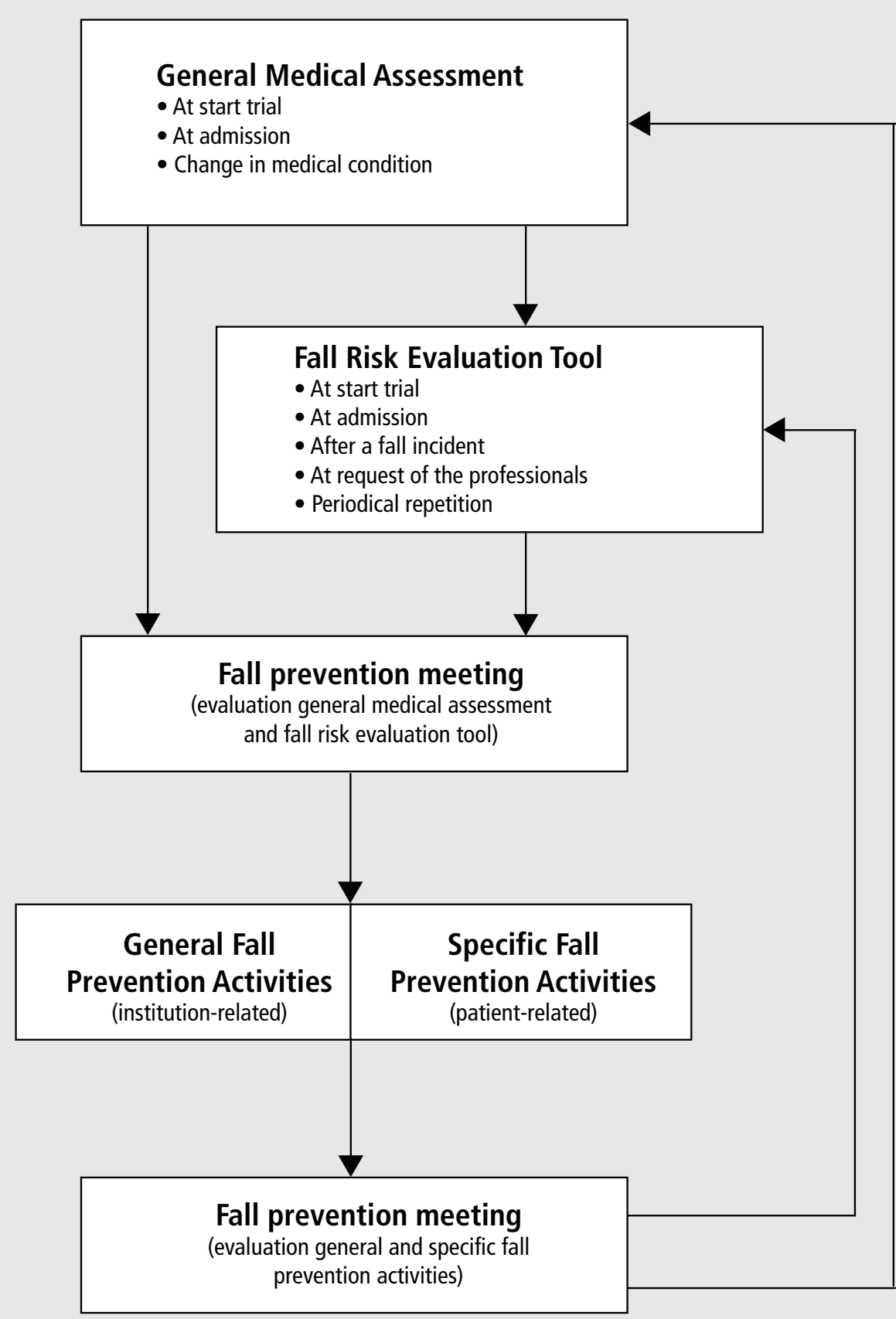




\section{Chapter 5}

the circumstances and causes of falls, critically reviewing and monitoring medication intake (type, number, dose, and time of intake), individually designed exercise programmes, carefully (re)assessing the need for assistive and protective aids, and promoting the correct use of these aids.

An occupational therapist also screened the main areas of each ward using a checklist for environmental hazards, e.g. lighting, floor surface and obstacles inside the facility.

Besides specific fall prevention activities, the team could also implement general fall prevention activities, such as staff training and creating a safe environment.

Figure 1 outlines the structure of the intervention programme.

\section{Data collection}

Data on falls and injurious falls were collected prospectively by asking all participating wards to keep records of any fall incident on a structured report form. ${ }^{18}$ At baseline, nursing home staff assessed the following characteristics of study participants: age, gender, Barthel ADL Index score, MMSE score, standing pattern and gait pattern (independent, using assistive device and/or professional assistance, impossible), total number of drugs used by patients, and the use of certain types of drugs (cardiovascular, psychotropic, antihistaminic, diabetes-related, and others). ${ }^{19,20}$ Data about the use of alarm devices and restraints (fixation, bedrails and other restraints) were also collected during the inclusion period.

In addition, we recorded the following information about the study wards: nursing staff manhours, and the number of falls and injuries on the study ward during the twelve months preceding the start of the trial. These data were collected retrospectively, using the register of falls that Dutch nursing homes are required to maintain. ${ }^{18}$

For each patient, we registered the starting and ending date of inclusion in the study, as well as the date of inclusion in the intervention programme which was the date that the fall prevention team discussed the results of the first fall risk evaluation. All patients residing on the study wards at the start of the inclusion period were automatically included in the study. Patients admitted in the course of the inclusion period were included on the day of admission. Inclusion in the study ended either on the last day of the inclusion period or, in the event of earlier discharge or a patient passing away, on the date of discharge or death.

\section{Statistical analysis}

Data were analysed with multilevel Poisson regression analysis. Multilevel 
analysis was used because of the hierarchical structure of the data (patients were clustered within wards) and Poisson regression was used because both outcome variables were 'counts'. All analyses were performed with MLwiN (version 1.1). ${ }^{21}$

Data were analysed according to the intention-to-treat principle, with adjustment for length of stay of the patient on the ward during the trial period of twelve months. To adjust for coincidental differences between the intervention group and the control group, an intention-to-treat analysis was performed with adjustment for ward-related parameters (nursing staff man hours per bed and number of falls per bed in the twelve months previous to the trial), length of stay on the ward during the inclusion period and other patient-related parameters (age, gender, Barthel ADL Index score, MMSE score, gait pattern, total number of drugs used, duration of use of alarm devices, and use of fixation, bedrails and other restraints during the inclusion period). In the analyses for the outcome parameter injurious falls, we adjusted for number of injuries per bed during the twelve months previous to the trial, instead of number of falls per bed. The variables with regard to standing pattern and use of certain types of drugs could not be added to the model because these multilevel models did not converge.

A subgroup analysis for the outcome parameter falls used only those patients in the intervention group who actually participated in the intervention programme and the entire control group. For the intervention group the value of the parameter "length of stay on the ward during the inclusion period" concerned the duration of inclusion in the intervention programme, and the value of the outcome variable concerned the number of falls during this period. Additional subgroup analyses were performed to estimate the effect of the intervention programme for different durations of participation in the intervention.

A significance level of 0.05 was maintained for all analyses.

\section{Results}

During twelve months of follow-up, 518 patients were included in the trial (269 in the control group and 249 in the intervention group). In the intervention group, the relatives/legal guardians of 229 patients agreed to the patient's participation in the intervention programme (92\%). Of this group, 200 patients were actually included in the programme. The mean duration of inclusion was 0.50 years (SD 0.28). Figure 2 shows the flow of study wards and participants. 


\section{Chapter 5}

Figure 2. Flow of nursing homes and patients

Nursing homes assessed for eligibility $(n=119)$

Nursing homes excluded $(n=85)$

Refused to participate $(n=45)$

Did not respond $(n=40)$

Eligible nursing homes $(n=34)$

- low fall rate $(n=13)$

- medium fall rate $(n=14)$

- high fall rate $(n=7)$

Randomly selected for participation $(n=12)$

- low fall rate $(n=4)$

- medium fall rate $(n=4)$

- high fall rate $(n=4)$

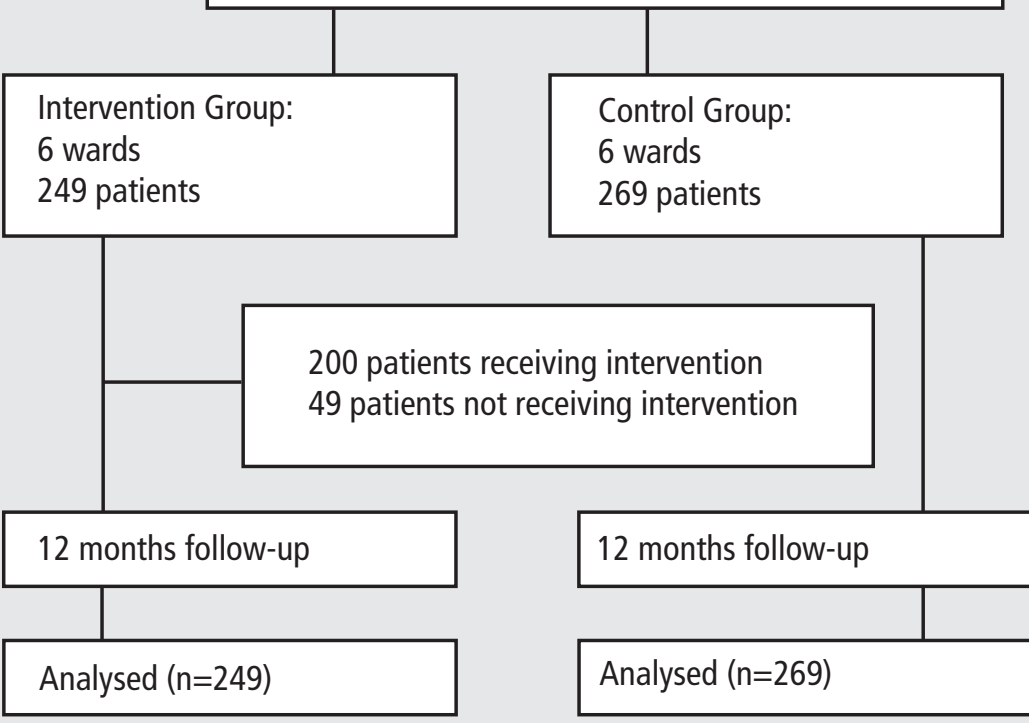


Table 1. Characteristics of Study Wards and Study Patients*

\begin{tabular}{|c|c|c|}
\hline & $\begin{array}{l}\text { Control group } \\
(n=262-269)^{\dagger}\end{array}$ & $\begin{array}{l}\text { Intervention group } \\
(\mathrm{n}=225-249)^{\dagger}\end{array}$ \\
\hline \multicolumn{3}{|l|}{ Ward characteristics } \\
\hline Number of beds (sum) & 171 & 177 \\
\hline Nursing staff manhours per bed & $0.67(.05)$ & $0.62(.04)$ \\
\hline \multicolumn{3}{|l|}{ Number of fall incidents per bed during the 12 months } \\
\hline previous to the inclusion period & $2.67(.87)$ & $2.91(1.43)$ \\
\hline \multicolumn{3}{|l|}{ Number of injuries as a result of falls, per bed, during the } \\
\hline 12 months previous to the inclusion period ${ }^{\ddagger}$ & $0.35(0.20)$ & $1.01(0.55)$ \\
\hline \multicolumn{3}{|l|}{ Patient characteristics } \\
\hline Age & $83.3(7.7)$ & $82.1(7.7)$ \\
\hline Gender ( $\%$ male) & $29 \%$ & $35 \%$ \\
\hline Barthel ADL Index score (0-20) & $8.6(6.5)$ & $8.1(6.5)$ \\
\hline MMSE score (0-29) & $7.0(6.6)$ & $9.3(7.8)$ \\
\hline \multicolumn{3}{|l|}{ Standing pattern } \\
\hline$\%$ independent & $50 \%$ & $42 \%$ \\
\hline$\%$ assistive device and/ or physical assistance & $28 \%$ & $39 \%$ \\
\hline$\%$ impossible & $22 \%$ & $19 \%$ \\
\hline \multicolumn{3}{|l|}{ Gait pattern } \\
\hline$\%$ independent & $40 \%$ & $32 \%$ \\
\hline$\%$ assistive device and/ or physical assistance & $29 \%$ & $32 \%$ \\
\hline$\%$ impossible & $31 \%$ & $36 \%$ \\
\hline Total number of drugs used & $4.97(3.06)$ & $4.75(3.39)$ \\
\hline \multicolumn{3}{|l|}{ Use of drugs per type } \\
\hline cardiovascular (\% use) & $57 \%$ & $47 \%$ \\
\hline psychotropic (\% use) & $65 \%$ & $66 \%$ \\
\hline antihistaminic (\% use) & $6 \%$ & $4 \%$ \\
\hline diabetes related (\% use) & $17 \%$ & $12 \%$ \\
\hline others (\% use) & $76 \%$ & $81 \%$ \\
\hline Length of stay on the ward during inclusion period in years & $0.62(0.39)$ & $0.68(0.34)$ \\
\hline Duration of use of alarm device during inclusion period in years & $0.14(0.33)$ & $0.11(0.25)$ \\
\hline \multicolumn{3}{|l|}{ Duration of use of restraints during inclusion period in years } \\
\hline fixation & $0.09(0.23)$ & $0.11(0.25)$ \\
\hline bedrails & $0.30(0.39)$ & $0.39(0.41)$ \\
\hline others & $0.07(0.23)$ & $0.08(0.25)$ \\
\hline
\end{tabular}




\section{Chapter 5}

Table 1 shows the characteristics of the control group and the intervention group. With regard to ward characteristics at baseline, the intervention wards had fewer nursing staff manhours per bed and a higher number of falls and injuries per bed during the twelve months preceding the inclusion period. With regard to patient characteristics, we observed only minor differences in the Barthel ADL Index scores, the MMSE scores, standing and gait pattern, use of drugs (type and total number) and length of stay on the ward during the inclusion period.

Falls

Table 2 shows the main results of the intervention study. During the inclusion period, 124 patients in the intervention group (50\%) and 122 patients in the control group (45\%) sustained one or more falls. Data showed 355 falls in 169.5 patient years in the intervention group $(=2.09$ falls per patient per year) and 422 falls in 166.3 patient years in the control group $(=2.54$ per patient per year). The intention-to-treat analysis with adjustment for length of stay on the ward during the trial period, showed that the mean fall incidence rate in the intervention group was lower than in the control group (rate ratio $=0.79$ ). This effect was not significant (95\% confidence interval $[\mathrm{Cl}]=0.43-1.47, \mathrm{p}=0.459$ ). The intention-to-treat analysis with adjustment for ward parameters (full time equivalents of nursing staff per bed and number of falls in the twelve months previous to the trial), length of stay on the ward during the trial period and the other patient-related parameters (age, gender, MMSE-score, Barthel ADL Index score, gait pattern, total number of drugs used, use of alarm devices and use of restraints), showed that the intervention group had a lower mean fall incidence rate than the control group (rate ratio $=0.64$ ). This effect was statistically significant.

The subgroup analysis, a comparison of the control group with those patients who participated in the intervention programme during their inclusion in it, reinforced this outcome (rate ratio $=0.54$ ). Further subgroup analysis, which took account of the duration of inclusion in the intervention programme, showed that the longer patients participated in the intervention, the lower the fall risk. These effects were all statistically significant.

\section{Injurious falls}

In the intervention group, there were 117 reported injurious falls in 169.5 patient years (0.69 injurious falls per patient per year); in the control group, 112 in 166.3 patient years ( 0.67 per patient per year). In the intervention group, seven injurious falls resulted in fractures, compared to three cases in the control group.

The intention-to-treat analysis with adjustment for length of stay on the ward 
during the trial period, showed no difference in fall incidence rate between both groups (rate ratio $=0.98,95 \% \mathrm{Cl}=0.56-1.70, \mathrm{p}=0.944$ ). The analysis with adjustment for patient parameters and ward parameters, showed that the intervention group had a lower incidence rate of injurious falls than the control group (rate ratio $=0.54$ ), but this difference was not statistically significant.

Table 2. Incidence of Falls and Injurious Falls during Inclusion Period in Intervention and Control

\begin{tabular}{|c|c|c|c|c|c|c|}
\hline & \multicolumn{2}{|c|}{ Control group } & \multicolumn{4}{|c|}{ Intervention group } \\
\hline & Patients (n) & $\begin{array}{l}\text { Falls per } \\
\text { patient } \\
\text { per year }\end{array}$ & $\begin{array}{l}\text { Patients } \\
\text { (n) }\end{array}$ & $\begin{array}{l}\text { Falls per } \\
\text { patient per } \\
\text { year }\end{array}$ & $\begin{array}{l}\text { Rate Ratio* } \\
(95 \% \mathrm{Cl})\end{array}$ & p-value \\
\hline
\end{tabular}

Intention-to-treat

analysis

$269 \quad 2.54$

$249 \quad 2.09$

$0.64(0.43-0.96) \quad 0.029$

Subgroup analyses for diverse periods

of inclusion in intervention programme:

$>0.0$ years

$\geq 0.3$ years

$\geq 0.5$ years

$\geq 0.7$ years

Injurious falls

Intention-to-treat

analysis

269

0.67

249

0.69

$0.54(0.29-1.01) \quad 0.054$

* adjusted for ward-related and patient-related parameters and for intra-cluster correlation, calculated with multilevel analyses (see statistical analysis)

\section{Discussion}

This study shows that the number of falls in psychogeriatric nursing home residents can be significantly reduced by a targeted multifactorial fall prevention intervention. The reduction in number of falls is substantial and of high clinical relevance. There are indications that the intervention may also reduce injurious falls, although this difference did not attain statistical significance.

The results of this trial are consistent with several comparable studies evaluating multidisciplinary, multifactorial fall prevention interventions and 


\section{Chapter 5}

provides new evidence of the effectiveness of multifactorial interventions in nursing homes. ${ }^{10,22-24}$

The present study has some limitations. Firstly, the power calculation was based on detecting a 30\% difference in falls, making this trial underpowered for detecting a significant difference on injurious falls. Secondly, there was a relatively large difference between the intervention and control group on the variable "number of injurious falls in the twelve months preceding the inclusion period". As a result of this, adjustment for this variable had a great influence on the estimated effect of the intervention on injurious falls. The large difference between the two groups may indicate that the intervention group consists of patients at higher risk for injurious falls. However it is also possible that these differences in injurious falls at baseline were only occasional fluctuations, which might have led to an overestimation of the effects on injurious falls. Thirdly, it is possible that we unintentionally selected the more highly motivated nursing homes for participation in our trial, because nursing homes that are not interested in fall prevention might not have agreed to participate in the trial. For this reason it remains unclear whether the programme would also be effective in homes where staff might be less motivated to tackle this problem. We must therefore regard the external validity of this study with reservation.

In conclusion, this study proves that fall prevention targeted at psychogeriatric patients in a nursing home setting is both possible and effective, but certainly not easy, e.g. the fall prevention teams required about 100 minutes per patient to complete the total intervention. Implementation of the programme in nursing home care is highly recommended. The intervention is also likely to be suitable for use in other settings like low-care residential homes for older people and geriatric wards in hospitals, but may need to be adapted to specific circumstances.

Finally, we recommend that future research should also seek to establish whether fall prevention has favourable effects on fall-related injuries. 


\section{References}

1. Perry BC. Falls among the elderly: A review of the methods and conclusions of epidemiologic studies. J Am Geriatr Soc 1982;30:367-371.

2. Rubenstein LZ, Josephson KR, Robbins AS (1994). Falls in the nursing home. Ann Intern Med 1994;121:442-451.

3. Dijcks BPJ, Neyens JCL, Schols JMGA, Haastregt JCM van, Crebolder HFJM, Witte LP de. Falls in nursing homes: on average almost two per bed, resulting in a hip fracture in $1.3 \%$. Ned Tijdschr Geneeskd 2005; 149:1043-1047.

4. Buchner DM, Larson EB. Falls and fractures in patients with Alzheimer-type dementia. JAMA 1987;257:1492-1495.

5. Vermeulen HBM. Falls and fall prevention in psychogeriatric nursing home patients. TV 1988.

6. Dijk van PTM, Meulenburg OGRM, Sande HJ van de et al. Falls in dementia patients. Gerontologist 1993;33(2):200-204.

7. Schoor NM van, Smit JH, Pluijm SMF et al. Different cognitive functions in relation to falls among older persons. Immediate memory as an independent risk factor for falls. J Clin Epidemiol 2002;55:855-862.

8. Morris JC, Rubin EH, Morris EJ et al. Senile dementia of the Alzheimer's type: an important risk factor for serious falls. J Gerontol 1987;42:412-417.

9. Brody EM, Kleban MH, Moss MS et al. Predictors of falls among institutionalised women with Alzheimer's disease. J Am Geriatr Soc 1984;32:77-82.

10. Gillespie LD, Gillespie WJ, Robertson MC et al. Interventions for Preventing Falls in Elderly People. The Cochrane Library, issue 2, 2004.

11. American Geriatrics Society, British Geriatrics Society and American Academy of Orthopaedic Surgeons Panel on Fall Prevention. Guideline for the Prevention of Falls in Older Persons. JAGS 2001;49:664-672.

12. Moreland J. A meta-analysis of fall prevention programs for the elderly: how effective are they? Nursing Research 2002;51(1):1-8.

13. Feder G, Cryer C, Donovan $\mathrm{S}$ et al. Guideline for the prevention of falls in people over 65. BMJ 2000;321:1007-1011.

14. Jensen J, Nyberg L, Gustafson $Y$ et al. Fall and injury prevention in residential care. Effects in residents with higher and lower levels of cognition. J Am Geriatr Soc 2003; 51:627-635.

15. Neyens JCL, Dijcks BPJ, Schols JMGA, Haastregt JCM van, Heuvel WJA van den, Crebolder HFJM, Witte LP de. Development and implementation of a multifactorial intervention for the prevention of falls and fall related injuries in psychogeriatric nursing home patients, a randomised controlled trial. TVV 2002;26:24-28.

16. Neyens JCL, Dijcks BPJ, Haastregt JCM van, Witte LP de, Heuvel WJA van den, Crebolder HFJM, Schols JMGA. The development of a multidisciplinary fall risk evaluation tool for demented nursing home patients in the Netherlands. BMC Public Health 2006;6:74. 


\section{Chapter 5}

17. Schols JMGA, Crebolder HFJM, Weel $C$ van. Nursing home and nursing home physician: the Dutch experience. J. Am Med Dir Assoc 2004, 5: 207-212.

18. Arcares. Registration of incidents and near accidents in nursing homes and old peoples' homes. Utrecht: Arcares, 2002.

19. Mahoney Fl, Barthel D. Functional evaluation: the Barthel Index. Md Med J 1965;14:61-65.

20. Folstein MF, Folstein SE, McHugh PR. Mini-Mental State: A practical method for grading the state of patients for the clinician, Journal of Psychiatric Research 1995;12:189-198.

21. Rasbash J, Browne W, Goldstein H, Yang M, Plewis I, Healy M, Woodhouse G, Draper D. A user's guide to MLwiN, 2nd Ed. London: Institute of Education, 1999.

22. Becker $\mathrm{C}$, Kron $\mathrm{M}$, Lindemann $\mathrm{U}$ et al. Effectiveness of a multifaceted intervention on falls in nursing home residents. J Am Geriatr Soc 2003;51:306-313.

23. Jensen J, Lundin-Olsson L, Nyberg L et al. Fall and injury prevention in older people living in residential care facilities. Ann Intern Med 2002;136:733-741.

24. Ray WA, Taylor JA, Meador KG et al. A randomised trial of a consultation service to reduce falls in nursing homes. JAMA 1997;278:557-562. 



\section{Chapter 6}

\section{Feasibility of a multifactorial fall prevention programme for psychogeriatric nursing home patients}

J.C.L. Neyens, M.H.C. Bleijlevens, B.P.J. Dijcks, J.C.M. van Haastregt, L.P. de Witte, J.M.G.A. Schols, W.J.A. van den Heuvel. 


\section{Chapter 6}

\section{AbStRACt}

\section{Aim}

The aim of this study was to assess the feasibility of a multifactorial intervention to prevent falls and related injuries in psychogeriatric nursing home patients.

\section{Background}

Falls are a major problem for elderly persons living in long-term care facilities, especially psychogeriatric patients. Falls may have severe consequences for health and quality of life. It is important, therefore, to prevent falls and related injuries among psychogeriatric nursing home patients.

\section{Method}

We developed a multifactorial intervention to prevent falls in psychogeriatric nursing home patients. The programme consisted of a general medical assessment focusing on fall risks and a specific fall risk evaluation applied by a multidisciplinary fall prevention team. Successively, general and individually tailored fall prevention activities were made on the basis of these assessment tools.

The intervention programme was executed in six psychogeriatric nursing home wards. Data were collected by means of semi-structured face-to-face interviews, self-administered questionnaires and examination of registration forms and patient files. In order to evaluate the feasibility of the intervention, we explored the following research questions: a) to what extent was the intervention carried out according to protocol; b) what factors may have influenced the implementation process; and c) how did ward staff rate the intervention.

\section{Findings}

We found that the intervention was not conducted entirely according to protocol in our study. The most common protocol deviations concerned the assessment of environmental fall risks and the recording of recommendations resulting from the assessments. Lack of time, extra workload and poor communication between nursing staff and fall prevention teams were reported to have hampered the implementation process. Factors, which may have positively influenced the implementation process, were the good communication between the different medical disciplines in the fall prevention team, the fall prevention meetings and the growing awareness of risk factors for falling. 


\section{Conclusion}

This process evaluation has shown that implementing a fall prevention intervention targeted at psychogeriatric patients in a nursing home setting is possible, but certainly not easy. Various improvements to the programme might facilitate implementation: a simpler recording system, standard establishment of a fall prevention team, more attention for communication in the protocol, the addition of an introductory information programme to the protocol, and a temporary increase in staffing. In view of the favourable effects of the programme on number of falls, we recommend implementing an improved version of the programme in regular nursing home care.

\section{Keywords}

fall prevention, psychogeriatric, process evaluation, nursing home, feasibility 


\section{INTRODUCTION}

Fall incidents are a major problem in independently living elderly and even more so in elderly persons residing in long-term care facilities. ${ }^{1-3}$ Falls may have severe consequences for health status and quality of life, especially when resulting in injury. ${ }^{4,5}$ In Dutch nursing homes, the fall incidence is almost two per bed per year, of which $1.3 \%$ results in a fracture. ${ }^{2}$ The incidence of fractures in Dutch psychogeriatric nursing homes patients ranges from 26.3 to 28.8 per 1000 beds per year. ${ }^{2}$ Psychogeriatric disorders, especially dementia, are a major risk factor for falling. ${ }^{6-8}$ Demented patients suffer gradually deteriorating mobility and a diminishing ability to recognize, judge and avoid hazards. About $55 \%$ of patients in Dutch nursing homes suffer from dementia and they are involved in $75 \%$ of all fall incidents occurring in nursing homes. ${ }^{9}$

\section{BACKGROUND}

Despite the magnitude of the problem of falls in psychogeriatric nursing home residents, there is only limited evidence for the effectiveness and efficacy of fall prevention among these patients. ${ }^{1,10}$ We developed a multifactorial fall prevention intervention for psychogeriatric nursing home patients, consisting of a general medical assessment and a specific fall risk evaluation executed by a multidisciplinary fall prevention team. ${ }^{11}$ Between November 2003 and March 2005, we conducted a randomized controlled trial on twelve psychogeriatric wards in twelve nursing homes in the Netherlands to evaluate the effects of the intervention programme on falls and injurious falls. Six wards were assigned to the intervention group and six wards to the usual care group. On the intervention wards, the fall prevention programme was carried out for a period of twelve months. During this period, these six wards included 177 beds and 249 patients. We found that the programme had a significant favourable effect on fall incidence. ${ }^{13}$ The trial also revealed a favourable effect on injurious falls, but this effect did not attain statistical significance. We concluded that a randomized controlled trial should always include a detailed process evaluation, as it is essential not only for assessing the effectiveness of an intervention but also its feasibility in daily practice. ${ }^{14}$ In order to assess the feasibility of our multifactorial intervention programme, we therefore evaluated the intervention process at the same time that we carried out the trial. In this paper, we discuss the results of our evaluation of the intervention process. 


\section{The Study}

\section{Aims}

The aim of this process evaluation was to assess the feasibility of our multifactorial fall prevention programme in daily nursing home care by assessing: a) the extent to which the intervention was performed according to protocol; b) factors that may have influenced the implementation process; and c) opinions of ward staff about the intervention programme.

\section{Design}

This process evaluation was a descriptive study with longitudinal elements. Quantitative and qualitative data were collected, some retro-spectively and others prospectively.

\section{The multifactorial fall prevention programme}

The intervention consisted of a general medical assessment and a specific fall risk evaluation tool (figure 1). Each intervention ward established its own multidisciplinary fall prevention team, consisting of a nursing home physician, two nurses, a physiotherapist and an occupational therapist. These teams were set up especially for the trial and coordinated the intervention during fall prevention meetings held each fortnight. Medical staff performed general medical assessments, which consisted of a medical history and a physical examination, when a patient entered the trial and whenever there was a change in medical condition. During the assessment, special attention was paid to identifying and addressing health-related risk factors for falling. The multidisciplinary fall prevention team executed the fall risk evaluation tool. They assessed the following risk factors for falls: fall history, medication intake, mobility, and choice and use of assistive and protective aids. ${ }^{12}$ An occupational therapist evaluated the main areas of the ward using a checklist for environmental hazards. Fall risk evaluations were performed when a patient entered the trial, after a fall, and at request of ward staff. There were at least two evaluations per year, even if there had been no fall incident or request. Newly admitted patients had to undergo both the general medical assessment and the fall risk evaluation within three days of admittance.

The fall prevention team discussed the outcomes of the fall risk evaluation in conjunction with the general medical assessment and decided which individual fall prevention activities were necessary. Team members or their colleagues on the ward then executed these individually tailored activities, which could include any or all of the following: anticipating the circumstances and causes of falls, critically 


\section{Chapter 6}

reviewing and monitoring medication intake (type, number, dose and time of intake), individually designed exercise programmes, carefully assessment of the need for assistive and protective aids, and promoting correct use of these aids. Besides specific activities, the team could also implement general fall prevention activities, such as staff training, creating a safe environment and using safe materials. The researchers contacted the fall prevention teams every fortnight and were always available for consultation. The different components of the fall prevention programme, i.e. composition of the fall prevention team, assessment instruments, registration forms and time frame, were described in the intervention protocol. Prior to the start of the trial, the programme had been tested in a pilot study in two nursing homes. An outline of the intervention programme is given in figure 1.

We used the following strategy to implement the intervention on each of the six participating wards. First, one of the researchers visited the nursing home manager to explain the intervention and obtain approval for participation in the trial. After management approval, one psychogeriatric ward that met the inclusion criteria was selected for participation in the trial. Inclusion criteria were: at least 30 beds and not already using a fall prevention protocol. At the start of the intervention period, a fall prevention team was formed on each intervention ward. Fall prevention team members were given information and training about executing the intervention programme and using the recording forms designed specifically for the intervention. Fall prevention teams were advised to hold fall prevention meetings twice a month during the intervention period, and to link up with the regular multidisciplinary meetings already being held on the ward. The rest of the nursing staff were told about the fall prevention programme at the start of the intervention period and received information during the intervention period at the general multidisciplinary meetings.

\section{Participants}

Data were supplied by members of the fall prevention teams: 6 nursing home physicians, 12 nurses, 6 physiotherapists and 5 occupational therapists. One home did not have a full-time occupational therapist on its staff.

Data were also collected from nursing staff on the participating wards $(n=180)$, and from one executive at each participating nursing home $(n=6)$.

\section{Data collection}

The following methods were used to collect data: semi-structured face-to-face interviews with members of the fall prevention teams, self-administered questionnaires filled in by nursing staff and semi-structured face-to-face interviews with one executive member of each nursing home. In order to limit 
Figure 1. Outline of the intervention programme

\section{General Medical Assessment}

- At start trial

- At admission

- Change in medical condition

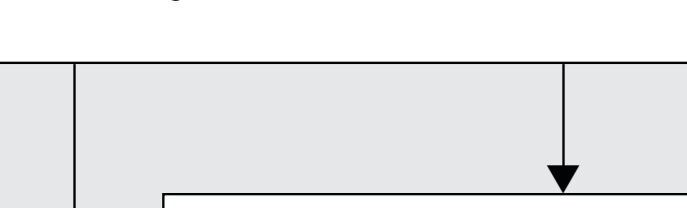

Fall Risk Evaluation Tool

- At start trial

- At admission

- After a fall incident

- At request of the professionals

- Periodical repetition

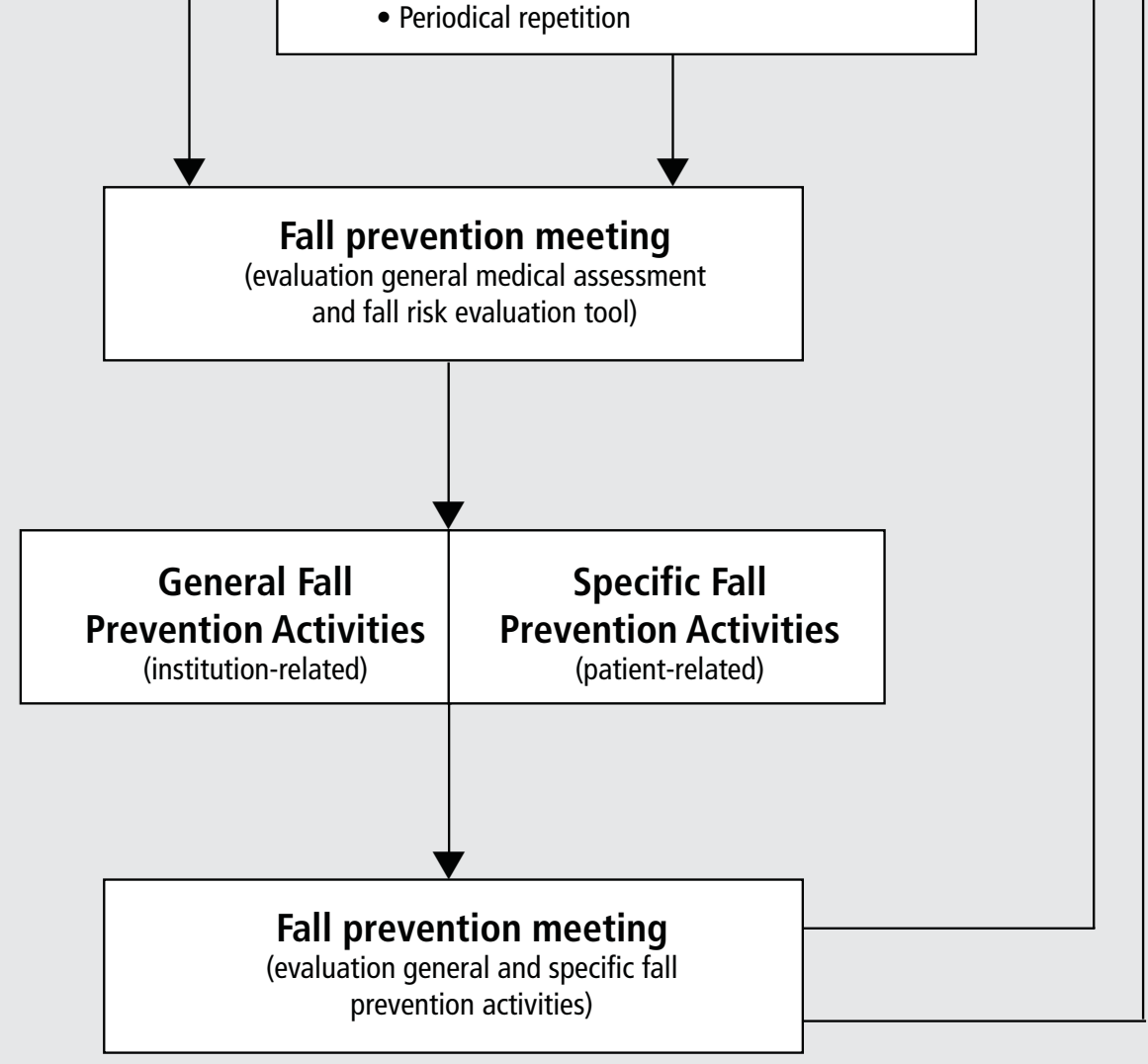




\section{Chapter 6}

the workload of nurses in the fall prevention teams, we interviewed only one of the two nurses in each team. Furthermore, we examined the recording forms of ten randomly selected patient files on each ward $(n=60)$. In table 1 , we show the different process outcomes and how they were measured.

Table 1. Outcome measures and measurement instruments

\begin{tabular}{|c|c|c|c|c|}
\hline \multirow[b]{2}{*}{ Outcome measures } & \multicolumn{4}{|c|}{ Measurement instruments } \\
\hline & $\begin{array}{c}\text { Interviews } \\
\text { fall prevention } \\
\text { team }\end{array}$ & $\begin{array}{l}\text { Questionnaire } \\
\text { nursing staff }\end{array}$ & $\begin{array}{l}\text { Interviews } \\
\text { nursing home } \\
\text { executives }\end{array}$ & $\begin{array}{l}\text { Recording } \\
\text { forms }\end{array}$ \\
\hline \multicolumn{5}{|l|}{ Execution intervention } \\
\hline \multicolumn{5}{|l|}{ - establishment and functioning } \\
\hline fall prevention team & $x$ & & & \\
\hline - execution of assessments & $x$ & & & $x$ \\
\hline \multicolumn{5}{|l|}{ - recommendations resulting from } \\
\hline the assessments & $x$ & & & $x$ \\
\hline \multicolumn{5}{|l|}{ Factors influencing } \\
\hline \multicolumn{5}{|l|}{ implementation process } \\
\hline - perceived barriers & $x$ & $x$ & & \\
\hline - perceived positive factors & $x$ & $x$ & & \\
\hline - organizational factors & & & $\mathrm{x}$ & \\
\hline \multicolumn{5}{|l|}{ Opinion about intervention programme } \\
\hline - training of fall prevention team & $x$ & & & \\
\hline - information for nursing staff & & $x$ & & \\
\hline - perceived workload & $x$ & $x$ & & \\
\hline - other feasibility issues & $x$ & & & $x$ \\
\hline
\end{tabular}

\section{Validity and reliability}

We had to develop tailor-made measurement instruments because no validated instruments were available to assess the process outcomes. Recording forms were pilot-tested in two nursing homes before the start of the study.

\section{Ethical considerations}

The study protocol was approved by the Medical Ethics Committee of the Institute for Rehabilitation Research. We obtained informed consent from the legal representatives of all patients whose recording forms and patient files were analysed. 


\section{Data analysis}

Quantitative data obtained from the questionnaires, recording forms and patient files were analysed by means of descriptive statistics. Qualitative data from the interviews were categorized according to matching contents of the answers.

\section{ResULts}

\section{Response}

We interviewed 24 of the 29 members of the fall prevention teams: 6 nursing home physicians, 7 nurses (in one home, both nurses wanted to participate in the interview), 6 physiotherapists and 5 occupational therapists.

Of the 180 self-administered questionnaires sent to nursing staff on the six intervention wards, 44 were returned, giving us a response rate of $24 \%$.

We also interviewed one executive member from each of the six nursing homes.

\section{Performance of the intervention according to protocol}

Establishment and functioning of fall prevention teams

At the start of the intervention, a multidisciplinary fall prevention team was established on each of the six intervention wards. Five teams were chaired by the nursing home physician, one team by the ward physiotherapist. One nursing home did not have a full-time occupational therapist, but team members could consult an occupational therapist if necessary.

Fifteen out of 24 fall prevention team members reported that it was difficult to hold fall prevention meetings twice a month, due to team members' conflicting schedules, different shifts and their regular care load. On five intervention wards, fall prevention meetings could not be linked up with the regular multidisciplinary meetings, as we had recommended.

\section{Execution of assessments}

Two assessment tools were used in the intervention: the general medical assessment and the fall risk evaluation tool. The actual execution of assessments was analysed by checking the recording forms of sixty randomly selected patients. For all 60 selected patients, the medical assessment was carried out (see Table 2), but as forms were not always filled out completely, it is uncertain whether all aspects of the medical assessment were performed. With regard to the fall risk evaluation tool, all components of the evaluation were performed in the majority of cases. 


\section{Chapter 6}

On more than $25 \%$ of forms, however, evaluators had omitted to give information on the Tinetti scale (Performance Orientated Mobility Assessment), high-risk behaviour and inspection of shoes. It is likely that these risk evaluation components were not

Table 2. Completeness of recording forms $(n=60)$ regarding assessments and recommendations

\begin{tabular}{|c|c|c|c|c|}
\hline & $\begin{array}{l}\text { Complete form } \\
\text { form }\end{array}$ & $\begin{array}{l}\text { Incomplete } \\
\text { form }\end{array}$ & $\begin{array}{l}\text { Empty } \\
\text { form }\end{array}$ & $\begin{array}{l}\text { Form } \\
\text { missing }\end{array}$ \\
\hline \multicolumn{5}{|l|}{ Assessments } \\
\hline \multicolumn{5}{|l|}{ General medical assessment } \\
\hline medical history & 52 & 8 & 0 & 0 \\
\hline physical examination & 43 & 17 & 0 & 0 \\
\hline \multicolumn{5}{|l|}{ Fall Risk Evaluation Tool } \\
\hline fall history & 50 & 7 & 3 & 0 \\
\hline medication & 59 & 0 & 1 & 0 \\
\hline Barthel score & 53 & 0 & 7 & 0 \\
\hline Tinetti score & 44 & 0 & 16 & 0 \\
\hline high-risk behaviour & 31 & 0 & 29 & 0 \\
\hline assistive aids & 46 & 7 & 6 & 1 \\
\hline inspection of shoes & 40 & 0 & 20 & 0 \\
\hline protective aids & 52 & 1 & 6 & 1 \\
\hline Environmental risk assessment & 27 & 10 & 23 & 0 \\
\hline \multicolumn{5}{|l|}{ Recommendations } \\
\hline \multicolumn{5}{|l|}{ Recommendations resulting from } \\
\hline general medical assessment & 31 & 4 & 25 & 0 \\
\hline \multicolumn{5}{|l|}{ Recommendations resulting from } \\
\hline \multicolumn{5}{|l|}{ fall risk evaluation tool } \\
\hline fall history & 28 & 0 & 32 & 0 \\
\hline medication & 25 & 0 & 35 & 0 \\
\hline motor functions & 23 & 3 & 34 & 0 \\
\hline assistive aids & 30 & 2 & 23 & 5 \\
\hline protective aids & 31 & 0 & 24 & 5 \\
\hline \multicolumn{5}{|l|}{ Recommendations regarding } \\
\hline general fall prevention activities & 6 & 0 & 54 & 0 \\
\hline
\end{tabular}


performed according to protocol for these patients. As for the environmental check, we found that the appropriate form had been filled out in less than half of cases. Clearly, this check was not routinely carried out. The intervention protocol required newly admitted patients to be evaluated within three days of admission, but executing both the general medical assessment and the fall risk evaluation tool at such short notice was often impossible.

In the intervention period, 23 out of the 60 patients selected fell at least once, with a total of 44 fall incidents in this group. According to the intervention protocol, the fall risk evaluation tool had to be carried out again after each fall, but there was no record in any of the examined patient files of this having been done. Yet fall prevention team members did assure us that all fall incidents were reported and discussed at fall prevention meetings.

\section{Recommendations resulting from assessments}

The outcome of the general medical assessment and the fall risk evaluation resulted in recommendations for reducing a patient's fall risk, made by the fall prevention team (table 2). These recommendations should have been noted on the recording forms, but this was done in only about half of cases, on average.

\section{Factors that may have influenced the implementation process}

\section{Perceived barriers}

The 24 members of the fall prevention teams named several factors that they felt negatively influenced implementation: the number of recording forms which had to be filled out; overlap in some of the forms; and the extra time needed to fill out the forms. These three factors posed a substantial burden for the teams. Over one-third of fall prevention team members $(n=9)$ reported feeling the increased workload as an important barrier to implementation.

\section{Perceived positive factors}

Fall prevention team members also mentioned factors which had a positive influence on implementation: good communication between professionals from different medical disciplines within the teams, fall prevention meetings, systematic recording, and involvement of the researchers. Team members were also motivated by their growing awareness of risk factors for falling and the fact that measures could be taken to reduce fall risk.

\section{Organizational factors}

Interviewed executive members of the six nursing homes all stated that no major changes had occurred in their organization (such as understaffing, management 


\section{Chapter 6}

changes, reorganization and insufficient financial resources with regard to fall prevention activities) that could have influenced the execution of the intervention programme. The executives did mention some understaffing, although not related to the intervention, in evening and night shifts, affecting supervision, and during the summer months, affecting execution of the intervention.

\section{Opinions about the intervention programme}

Training of fall prevention team

At the start of the intervention, members of the fall prevention teams were given information and training about the intervention protocol. The quality of instruction was positively evaluated by 15 out of 24 fall prevention team members. Twenty agreed that the training provided complemented with their knowledge on the subject and eleven members stated that the instructions were useful for their daily work.

\section{Information for nursing staff}

Forty-one out of 44 nursing staff members who responded to the questionnaire confirmed that they had been informed about the intervention. More than twothirds $(n=30)$ knew about the fall prevention team. Only one-third $(n=14)$ of respondents reported that the fall prevention programme was discussed during regular multidisciplinary meetings as prescribed in the protocol.

\section{Perceived workload}

Fall prevention teams reported that application of the fall risk evaluation tool required 15 to 25 minutes per medical discipline per patient. In total, the tool meant about 90 minutes of extra work per patient. Discussing and evaluating patient outcomes at the fall prevention meetings took, on average, 19 minutes per patient.

A small number of nursing staff experienced an increased workload $(n=7)$ or felt that fall prevention activities took up more time $(n=5)$.

\section{Other feasibility issues}

All 24 fall prevention team members confirmed that they had found the fall prevention meetings to be useful. Nursing home physicians stated that it was often impossible to execute both the general medical assessment and fall risk evaluation tool within three days of admittance. Five days seemed a better margin. Twenty fall prevention team members reported that the recording forms were impractical. There were too many forms to fill out and some overlapped considerably. Often, the form on 'general fall prevention activities' was not filled out because it was considered to be ward-related. 
Communication between fall prevention teams and nursing staff was assessed as 'not good' by 16 members of the teams. Nursing staff also perceived inadequate communication with the fall prevention team, especially regarding the different fall prevention activities prescribed in the course of the intervention.

Overall, all team members were satisfied with the medical assessment, fall risk evaluation tool and fall prevention meetings. Fourteen members thought that the protocol could be implemented in daily practice. Nursing staff, however, felt they had insufficient time to execute the intervention in its entirety. All in all, members of the fall prevention teams rated the programme positively. Problems were reported and observed with regard to communication between fall prevention teams and nursing staff, recording forms, and timing of assessments.

\section{Discussion}

In this study, we evaluated the feasibility of a multifactorial fall prevention programme in psychogeriatric nursing home patients. Although the intervention was not carried out entirely according to protocol, it was found to be effective. ${ }^{13}$ Protocol deviations were mainly observed with regard to the assessment of environmental risks and the recording of recommendations resulting from fallrelated assessments. Lack of time, extra workload and poor communication between nursing staff and fall prevention team were reported to have hampered the implementation process. Factors, which may have positively influenced the implementation process, were the good communication and strong cooperation between the different disciplines of the fall prevention team, their high motivation and the fall prevention meetings. Based on the results of this process evaluation, we conclude that the fall prevention programme in its present form is feasible but certainly eligible to further improve the implementation of the programme.

\section{Study limitations}

This study had a number of limitations. For practical reasons, we only assessed recording forms of 60 patients (10 in each home) and not of all 249 patients. Another shortcoming was the low response to the written questionnaire for ward staff. Questionnaire outcomes might therefore not be representative for the total population of nurses on the wards. The questionnaire results should be interpreted with care. To enhance successful implementation, researchers 
contacted fall prevention teams twice a month, either paying a visit, or telephoning or e-mailing. This was much appreciated by team members and may have had a positive effect on executing the intervention. However, when the programme is implemented in regular care, this level of support will not be routinely available. It is possible that there would have been more protocol deviations without this support.

\section{Main findings}

There are several explanations for the difficulties encountered in carrying out the intervention entirely according to protocol. First, although none of the nursing home managers reported understaffing, nursing staff themselves did report that the intervention increased their workload, which was already high. Second, the programme's rather complex recording system may have negatively influenced the implementation process. The forms were apparently not well adapted to regular nursing home recording practice. One of the reasons for this might be that some forms had to be filled in by different medical professionals, which may have been impractical and caused delays. In other cases, there were overlaps between forms. Furthermore, the fact that implementation was combined with research increased the number of forms which had to be filled out, as researchers needed additional information for their process evaluation.

The difficulty of linking up fall prevention meetings and the regular multidisciplinary meetings may also have been an important barrier to implementation. This opened the way to misunderstandings between the fall prevention team and the rest of ward staff and negatively affected compliance among those responsible for executing the intervention. Consequently, the programme might not have been executed as well as it could have been, in particular the recommendations of the fall prevention teams. Perhaps the intervention would have been even more effective with improved implementation. ${ }^{14}$

The poor communication between fall prevention teams and nursing staff may also have increased the deviations from protocol. Poor communication may have been due to general time constraints, personnel changes due to shift work, and the difficulty of linking up fall prevention meetings with the existing multidisciplinary meetings, as mentioned above.

Finally, nursing staff received insufficient information and training before implementation. As a result, they may have been less motivated and given a suboptimum effort on the intervention. 
In conclusion, this process evaluation proves that it is possible to implement a fall prevention intervention targeted at psychogeriatric patients in a nursing home, but certainly not easy, especially in tightly staffed healthcare facilities. The randomized controlled trial showed that the intervention was more effective when implemented over a longer period of time. In view of this and the results of the process evaluation, we recommend making further improvements to the intervention programme with regard to its applicability in normal nursing practice. The first step should be a simplification of the recording system for assessments. The recording of fall prevention activities should be integrated in existing patient files. Second, we recommend that fall prevention teams are established, so that they can coordinate at least the start of the implementation process. We also recommend paying more attention to improving communication between the professionals directly involved with the intervention programme (fall prevention team members) and those who are more indirectly confronted with the programme in daily practice (nursing staff). A more fleshed out training and information programme should be an integral part of the intervention programme. Finally, when implementing this intervention, nursing homes are advised to plan a temporary increase in staff because the intervention will certainly increase their workload, especially in the early stages. ${ }^{15}$

In view of the favourable effects of this multifactorial intervention programme on preventing falls, we recommend that it is implemented in regular nursing home care. It is likely to be suitable for use in other settings as well, such as low-care residential homes for older people and geriatric wards in hospitals, although it may need to be adapted to specific circumstances. 
Chapter 6 


\section{References}

1. Gillespie LD, Gillespie WJ, Robertson MC,Lamb SE, Cumming RG, Rowe BH. Intervention for preventing falls in elderly people. Cochrane Database Syst Rev 200;3: CD000340.

2. Dijcks BPJ, Neyens JCL, Schols JMGA, van Haastregt JCM, Crebolder HFJM, de Witte LP. Valincidenten in verpleeghuizen: gemiddeld bijna 2 per bed per jaar met bij 1,3\% een fractuur als gevolg. Ned Tijdschr Geneeskd 2005, 149: 1043-7.

3. Baker SP, Harvey AH. Fall injuries in the elderly. Clin Geriatr Med 1985; 1: 501-12.

4. O'Loughlin JL, Robitaille Y, Boivin J, Suissa S. Incidence of risk factors for falls and injurious falls among the community-dwelling elderly. Am J Epidemiol 1993; 137: 342-54.

5. Tinetti ME, Speechley M, Ginter SF. Risk factors for falls among elderly persons living in the community. N Engl J Med 1988; 319: 1701-7.

6. van Doorn C, Gruber-Baldini AL, Zimmerman S, Hebel JR, Port CL, Baumgarten M, et al. Dementia as a Risk Factor for Falls and Fall Injuries Among Nursing Home Resident. JAGS 2003;51:1213-1218.

7. Buchner D, Larson E. Falls and fractures in patients with Alzheimer-type dementia. JAMA 1987;257:1492-1495.

8. Jensen J, Nyberg L, Gustafson Y, Lundin-Olsson L. Fall and Injury Prevention in Residental Care-effects in Residents with Higher and Lower Levels of Cognition. JAGS 2003;51:627-635.

9. Schols JMGA, Crebolder HFJM, Weel $C$ van. Nursing home and nursing home physician: the Dutch experience. J Am Med Dir Assoc 2004, 5: 207-212.

10. Neyens JCL, Haastregt JCM van, Dijcks BPJ, Heuvel WJA van den, Witte LP de, Schols JMGA. Effectiveness and feasibility of interventions for preventing falls in elderly people in long-term care facilities: a systematic review. Submitted

11. Neyens JCL, Dijcks BPJ, Schols JMGA, Haastregt JCM van, Heuvel WJA van den, Crebolder HFJM, Witte LP de. Ontwikkeling en implementatie van een multifactoriële interventie gericht op de preventie van valincidenten en de gevolgen daarvan bij psychogeriatrische verpleeghuispatiënten. TVV 2002, 26: 24-28.

12. Neyens JCL, Dijcks BPJ, Haastregt JCM van, Witte LP de, Heuvel WJA van den, Crebolder HFJM, Schols JMGA. The development of a multidisciplinary fall risk evaluation tool for demented nursing home patients in the Netherlands. BMC Public Health 2006, 6: 74.

13. Neyens JCL, Dijcks BPJ, Twisk JW, , Schols JMGA, Haastregt JCM van, Heuvel WJA van den, Witte LP de. A multifactorial intervention for the prevention of falls and related injuries in psychogeriatric nursing home patients, a randomised controlled trial (RCT). Submitted.

14. Oakley A, Strange V, Bonell C, Allen E, Stephenson J, RIPPLE Study Team. Process evaluation in randomised controlled trials of complex interventions. BMJ 2006; 332: 413-6.

15. Schnelle JF, Alessi CA, Simmons SF, AI-Samarrai NR, Beck JC, Ouslander JG. Translating clinical research into practice: a randomized controlled trial of exercise and incontinence care with nursing home residents. J Am Geriatr Soc 2002; 50: 1476-83. 



\section{Chapter 7}

\section{CBO guidelines to prevent falls}

in the elderly:

\section{how can they be used in the institutionalised elderly?}

J.C.L. Neyens, B.P.J. Dijcks, A. Kinkelder de, W.C. Graafmans, J.M.G.A. Schols. 


\section{Chapter 7}

\section{Abstract}

Fall incidents are common in community-dwelling elderly and even more so in institutionalised elderly. Fall-related research data indicate that multifactorial interventions focused on preventing falls and fall-related injuries can have positive effects.

The guidelines 'Prevention of fall incidents in the elderly' developed by the Dutch Institute for Healthcare Improvement (CBO) was published in November 2004. The guidelines address risk factors for falling and the prevention of fall incidents in different healthcare settings. The main points for nursing homes are:

- all nursing home patients are at risk;

- fall prevention activities should be based on fall risk assessments carried out for all nursing home patients;

- specific fall prevention activities, tailored to the patient's situation, as well as general fall prevention activities at institutional level are necessary;

- a multifactorial approach is preferred and should be implemented by multidisciplinary teams. 


\section{INTRODUCTION}

Fall incidents occur frequently in elderly people. One-third of community dwelling elderly aged 65 and over, fall at least once a year. In old people's homes and nursing homes, half of all residents fall at least once a year. ${ }^{1}$ The international annual fall incidence in nursing homes is, on average, 1.5 per bed (range from 0.2 to 3.6). ${ }^{2}$ In a survey of 202 Dutch nursing homes to determine the fall incidence in 2000 and 2001, the mean annual fall incidence was almost two per bed, with the highest occurrences on psychogeriatric wards. ${ }^{3}$ The group of somatic patients had an annual incidence, on average, of 1.3 per bed, compared to a mean fall incidence of 2.2-2.3 per bed for psychogeriatric patients. While all nursing home patients are at risk of falling, the $55 \%$ of patients suffering cognitive decline, such as dementia, are involved in $75 \%$ of fall incidents in nursing homes. ${ }^{3}$

Falls can have physical, psychological and social consequences. About $10 \%$ of falls in the elderly result in serious injuries. The risk of a hip fracture is higher among long-term care residents compared to community-dwelling elderly. ${ }^{4}$ Of all fall incidents in Dutch nursing homes, $1.3 \%$ result in fractures, with a total of 1500 fractures per year. ${ }^{3}$ The main psychological consequences are fear of falling, fear of moving around (loss of confidence) and decreased participation in social activities. Clearly, fall incidents increase the vulnerability of nursing home patients.

Finally, it is also necessary to take account of the injury-related healthcare costs after a fall, e.g. admission to hospital. Falls often increase the need for care, especially when resulting in hip fracture, and increase the workload of nursing staff.

Fall incidents and their consequences can have a substantial negative influence on the autonomy and quality of life of the patients involved and can lead to increased morbidity, mortality and medical consumption. ${ }^{2,6}$

Various fall prevention activities are being undertaken in Dutch nursing homes, but it is still unclear how intended goals are to be reached. Research shows that fall prevention protocols in Dutch nursing homes are scarce. ${ }^{3}$

National evidence-based guidelines specifically for fall prevention in the elderly were not available until recently. In view of the possibly serious consequences of a fall, there is a need for effective fall prevention activities. Moreover, the Dutch Health Care Inspectorate (IGZ) requires nursing homes to pay more attention to fall problems. In the near future, the Dutch Health Care Inspectorate will use fall incidence in nursing homes as an indicator of the 
quality of care. It is therefore important for long-term care institutions to develop adequate fall prevention policy.

In November 2004, the Dutch Institute for Healthcare Improvement (CBO) published a national guidelines titled 'Prevention of fall incidents in the

elderly'. ${ }^{7}$ This guidelines incorporates the latest evidence-based knowledge with regard to fall prevention in the elderly for different healthcare settings (in the community, in hospitals, nursing homes and old people's homes). The guidelines address the following issues:

- extent and consequences of fall problems in these settings;

- the extent to which fall risk factors specifically apply to these settings;

- case finding;

- effectiveness of fall prevention interventions.

The guidelines also provide recommendations for daily practice. In this article we describe essential components of the guidelines for nursing homes.

\section{Development of the guidelines}

Development of the guidelines was initiated by the Dutch Clinical Geriatric Society (NVKG), incorporated in the 'Development and implementation of medical-specialist guidelines' programme of the Board of Medical Specialists and realised in collaboration with the $\mathrm{CBO}$, which provided procedural, methodical and secretarial support.

The new guidelines were to be based on scientific evidence. To that end, a literature search was performed in Medline, Cochrane, Embase, Psychlit, Sociofile and CINAHL covering the period January 1986 to July 2003. Previously published guidelines and articles from the reference lists were included in the search as well. When there was a lack of evidence, members of the CBO working group drew up conclusions based on expert opinions.

The process of guidelines development followed the usual CBO methodology and resulted in conclusions and recommendations per healthcare setting: in the community, hospitals, nursing homes and old people's homes. ${ }^{8}$ For an exhaustive discussion of the considerations that led to a specific recommendation, we refer the reader to the $\mathrm{CBO}$ guidelines. ${ }^{7}$

The following paragraphs contain the most important findings, conclusions and recommendations of these guidelines with regard to nursing homes. In general, these findings are also valid for old people's homes in the Netherlands. 


\section{Fall risk factors in nursing homes}

In order to adequately prevent fall incidents in nursing home patients, it is important to determine, first, the specific fall risk factors for this population. The literature describes many risk factors, which fall into two main categories: intrinsic risk factors, which can be attributed to the patient's state or condition, and extrinsic risk factors, which can be attributed to the patient's environment. Intrinsic risk factors are, in general, more important than extrinsic factors with regard to prevention.

In Table 1, we present fall risk factors found to be independent (i.e. adjusted for the effects of other factors) in at least two different studies. They are ranked according to diminishing strength of evidence. The data show that previous fall history is a very good indicator for future falls. Mobility impairments and disturbed balance are very important risk factors, as well as independence during transfers, psychotropic drugs and cognitive problems.

In Table 2, we present risk factors which were found to be independent in only one study. Caution should be exercised in interpreting these factors as fall risk factors.

Finally, our literature search produced factors which were described as risk factors, but were not adjusted for the effects of other risk factors (see Table 3 ). Future research will have to determine the scientific value of these possible risk factors.

Table 1. Independent fall risk factors for long-term care residents

\begin{tabular}{|c|c|}
\hline Risk factor & OR/RR/DR \\
\hline Previous falls $s^{1,18-22}$ & $1.8-7.6$ \\
\hline Mobility impairments and disturbed balance ${ }^{1,18,20,21.23-25}$ & $1.1-6.4$ \\
\hline Dependent in activities of daily living ${ }^{1,20,21}$ & $1.2-2.9$ \\
\hline $\operatorname{Age}^{18,20,22}$ & $1.1-3.8$ \\
\hline Independent transfer ${ }^{20,22}$ & $1.4-4.0$ \\
\hline Orthostatic hypotension ${ }^{1,26}$ & $2.0-2.1$ \\
\hline Psychotropic drugs $s^{18,23,27}$ & $1.7-7.6$ \\
\hline Dementia and cognitive decline $e^{1,18}$ & $2.4-6.2$ \\
\hline Behavioural problems ${ }^{19,21}$ & $1.6-1.9$ \\
\hline Heart medication ${ }^{1,18,22}$ & $2.9-3.0$ \\
\hline
\end{tabular}




\section{Chapter 7}

Table 2. Fall risk factors, proven independent in one study

\begin{tabular}{|c|c|}
\hline Risk factor & OR/RR \\
\hline Stroke $^{1}$ & 3.4 \\
\hline Decreased strength $^{24}$ & 5.9 \\
\hline Diabetes mellitus ${ }^{18}$ & 4.1 \\
\hline Male gender ${ }^{20}$ & 1.1 \\
\hline Disturbed vision ${ }^{24}$ & 6.7 \\
\hline Dizziness $^{1}$ & 2.1 \\
\hline Walking aids ${ }^{20}$ & 1.4 \\
\hline Change in everyday surroundings in the previous 2 years ${ }^{24}$ & 8.8 \\
\hline Oral bronchia dilator ${ }^{18}$ & 5.6 \\
\hline Residing $>2$ years in a LTC setting ${ }^{19}$ & 4.8 \\
\hline Wandering behaviour ${ }^{20}$ & 1.8 \\
\hline
\end{tabular}

Table 3. Potential fall risk factors for long-term care residents

\begin{tabular}{ll}
\hline Hearing problems & Polypharmacy \\
NSAID medication & Mood swings \\
Cardiovascular disease & Decreased sense of vibration \\
Low BMI & Neurological diseases \\
Gender & Parkinson disease \\
Hypertension & Dermatological disease \\
Arthritis & Bad personal impression of health \\
Hormones/metabolism medication & Fear of falling \\
Depression & Single \\
Analgesics & Weight loss \\
Incontinence & COLD \\
Gastro-intestinal medication & Restraints \\
Medication for Parkinson's disease & Foot abnormalities \\
Gastro-intestinal disease & Limited mobility in joints
\end{tabular}

\section{Fall risk assessment}

Since most nursing home patients have a combination of several, mostly intrinsic, risk factors for falling, we may conclude that they nearly all have a heightened fall risk. Totally immobile patients are probably the only exception. Fall incidents in this group of patients are mostly due to extrinsic factors, e.g. improper transfer technique or restraints.

Case finding in nursing homes (looking for patients with increased fall risk) is not 
meaningful because the entire nursing home population is at risk. Instead, a fall risk assessment of each patient is recommended and the outcomes should be recorded in their care plans to facilitate tailor-made fall prevention activities. At present, there is no specific instrument for use by nursing home staff that instantly provides relevant information with regard to fall risk. This information must be obtained by practical means. The guidelines recommend the strategic use of known information, e.g. medical history and status, and previous assessments.

A fall risk assessment should be repeated periodically and whenever there is a change in the patient's medical condition. Table 4 presents the risk factors that the $\mathrm{CBO}$ working group considered to be important and modifiable, and therefore eligible to be included in fall risk assessments.

Table 4. Survey of items eligible in a fall risk assessment

\section{Medical history}

- high-risk behaviour; an imbalance in performing activities and the physical and/or psychological capacities to carry out these activities safely

- bodyweight; undesired weight loss (at least $5 \%$ in one month or at least $10 \%$ in six months) indicates deteriorating general condition and is often linked with increased fall-related injury

- sight problems; deteriorating sight increases the fall risk

- hearing problems; in principle, bad hearing increases the fall risk (e.g. panic reactions)

- cardiovascular diseases; many cardiovascular diseases can lead to orthostatic hypotension and dizziness and thus increases the fall risk

- neurological diseases; most neurological diseases make standing and walking difficult and thus increases the fall risk

- urine incontinence; (nocturnal) need influences the walking pattern/behaviour and thus increases the fall risk

- mobility disorders can limit the function of standing and walking and thus increase the fall risk

- cognition; cognitive decline increases the fall risk

- fall history

- psychotropic medication

- environment (e.g. lighting, thresholds)

\section{Physical diagnostic items}

- illness of the patient

- body weight (at time of examination)

- blood pressure

- heart rate; disturbed frequency and rhythm indicate cardiovascular diseases

- ability to stand and walk

- disturbed vision

- footwear: absence, incorrect use of footwear and use of bad footwear increase the fall risk 


\section{Chapter 7}

The authors of this article recommend that a fall risk evaluation in nursing home patients should include at the very least fall risk factors out of Table 1. In view of the diverse nature of these risk factors, an evaluation should be multidisciplinary. In other words, medication should be assessed by the nursing home practitioner, nursing staff should monitors patients' fall risk through observation, the physiotherapist should focus on balance and mobility and the occupational therapist on environmental factors.

\section{Fall prevention activities}

The aim of fall prevention is to minimise the risk of falls and related injuries. Since all nursing home patients are at risk of falling and since many factors can cause a fall, it is essential to adopt general fall prevention measures, tailored to the institution's environment, to decrease the fall risk for all patients at institutional level. Such activities concern general safety policy, e.g. architectural and interior design, and staff awareness of the problem. The core of these measures may consist of:

1) continuous vigilance to prevent fall incidents:

- education and instruction for nursing home staff;

- permanent attention for fall prevention in daily care;

- a satisfactory staffing schedule ensuring sufficient supervision at all times;

- safe lifting and transfer techniques;

- safe use of assistive and protective devices;

- clear information about what to do after a fall;

- a strict, precise and complete registration of fall incidents and near-accidents and a fall prevention policy based on this registration.

2) a safe environment:

- sufficiently spacious and safe walking areas;

- handrails at strategic points;

- good lighting;

- adequate alarm systems;

- a familiar and recognisable environment;

- safe furniture;

- safe assistive and protective devices.

Besides general fall prevention measures, it is important to provide specific fall prevention activities, tailored to the patient's needs, and to record these in individual care plans. Specific patient-related activities can be based on a 
previous fall risk evaluation. Multifactorial fall prevention programmes have been shown to be more effective and are therefore recommended. Important aspects are:

- well-considered prescription of walking aids, correct maintenance and use to secure safe mobility and decrease the risk of falling;

- treatment of orthostatic hypotension incorporated in regular care;

- repeated critical evaluation of prescribed medication;

- tailor-made exercise programmes for each individual with regard to balance and strength;

- a bed alert system can be a useful aid to prevent fall incidents; ${ }^{10}$

- treatment of disturbed vision, e.g. (individual) aids and environmental adjustments;

- adequate footwear should be considered.

As falls can still occur regardless of all efforts, measures should also be taken to prevent or limit the severity of fall-related injuries. Preventing physical injuries usually means preventing fractures, for example by using external hip protectors. These are proven effective in preventing hip fractures, provided nursing home staff ensures they are fitted correctly. ${ }^{11,12}$

It is unclear whether vitamin D supplements for the elderly decrease the fracture incidence. The $\mathrm{CBO}$ guidelines 'Osteoporosis' recommends vitamin D supplements for elderly who do not go outdoors. ${ }^{13}$

Restraints are not realistic fall prevention measures. It has been shown that the use of restraints can be limited without a clear increase in the number of falls and fall-related injuries. ${ }^{14-16}$ Restraints are probably appropriate for a certain group of patients, but for other reasons than fall prevention.

\section{Implementation}

The CBO guidelines also give advice on implementation. ${ }^{7}$ According to the authors of this article, successful implementation in the nursing home branch requires:

- that the management team implements and facilitates a multifactorial multidisciplinary approach to fall problems;

- educational programmes and instructions, to be repeated each year;

- a fall prevention policy based on the registration of fall incidents;

- integration of fall prevention programmes in the daily care process;

- clearly formulated, delimited, and coordinated professional-related fall prevention tasks;

- feasible and practicable tasks, e.g. sufficient means and trained staff. 


\section{DisCUSSION}

The $\mathrm{CBO}$ guidelines 'Prevention of fall incidents in the elderly' helps nursing homes focus their attention on preventing falls and fall-related injuries in a qualitatively well-founded way. It must be noted, however, that only a few studies have been conducted into fall risk factors in nursing home settings and that these all involved small populations. This might have led to less reliable fall risk ratios. Future research should provide extra insight into some risk factors.

It is now up to nursing home executive boards to establish effective fall prevention policy. But simply implementing a fall prevention programme is not enough. The multifactorial multidisciplinary approach demands coordination, continuity and specific tasks for each discipline involved.

Data on favourable effects on fall-related injuries, and on economic costs and benefits are scarce. It is both important and necessary that further research is carried out into the effectiveness and applicability of fall prevention protocols in nursing homes. Currently, some of the authors are conducting a study into the effectiveness and feasibility of a new multifactorial multidisciplinary fall prevention intervention for psychogeriatric nursing home patients. ${ }^{17}$ The results are expected at the end of 2005. 


\section{References}

1. Graafmans WC, Ooms ME, Hofstee HMA, Bezemer PD, Bouter LM, Lips P. Falls in the elderly: a prospective study of risk factors and risk profiles. Am J Epidemiol 1996; 143 : 1129-36.

2. Rubenstein LZ, Josephson KR, Robbins AS. Falls in the nursing home. Ann Intern Med 1994; 121: 442-51.

3. Dijcks BPJ, Neyens JCL, Schols JMGA, van Haastregt JCM, Crebolder HFJM, de Witte LP. Falls in nursing homes: on average almost two per bed, resulting in a hip fracture in 1.3\%. Ned Tijdschr Geneeskd 2005; 149: 1043-7.

4. Ooms ME, Vlasman P, Lips P, Nauta J, Bouter LM, Valkenburg HA. The incidence of hip fractures in independent and institutionalized elderly people. Osteoporosis Int 1994; 4: 6-10.

5. Tinetti ME, Mendes de Leon CF, Doucette JT, Baker DI. Fear of falling and fall-related efficacy in relationship to functioning among community living elders. J Gerontol 1994; 49: M140-7.

6. American Geriatrics Society, British Geriatrics Society \& American Academy of Orthopaedic Surgeons on Falls Prevention. Guidelines for the prevention of falls in older persons. J Am Geriatr Soc 2001; 49: 664-72.

7. $\mathrm{CBO}$, Dutch Institute for Healthcare Improvement. Guidelines "Fall Prevention in the Elderly". Alphen aan den Rijn: Van Zuiden Communications BV, 2004.

8. Guidelines for $\mathrm{CBO}$ working groups (www.cbo.nl).

9. Arcares. Registration of incidents and near accidents in nursing homes and old peoples' homes. Utrecht: Arcares, 2002.

10. Vermeulen HBM. Falls and fall prevention in psychogeriatric nursing home patients. TVV 1988.

11. Gillespie LD, Gillespie WJ, Robertson MC, Lamb SE, Cumming RG, Rowe BH. Intervention for preventing falls in elderly people. Cochrane Database Syst Rev 2001; 3: CD000340.

12. Meyer G, Warnke A, Bender R, Muhlhauser I. Effect on hip fractures of increased use of hip protectors in nursing homes: cluster randomised controlled trial. BMJ. 2003 Jan 11; 326(7380): 76.

13. CBO, Dutch Institute for Healthcare Improvement. Guidelines "Osteoporose". Second edition. Alphen aan den Rijn: Van Zuiden Communications BV, 2002.

14. Evans LK, Strumpf NE, Allen Taylor SL, Capezutti E, Maislin G, Jacobsen B. A clinical trial to reduce restraints in nursing homes. J Am Geriatr Soc 1997; 45: 675-681.

15. Capezuti E, Strumpf N, Evans L, Maislin G. Outcomes of nighttime physical restraint removal for serverly impaired nursing home residents. Am J of Alzheimer's disease 1999; 14: 157- 164 . 


\section{Chapter 7}

16. Neufeld RR, Libow LS, Foley WJ, Dunbar JM. Cohen C, Breurer B. Restraint reduction reduces serious injuries among nursing home residents. J Am Geriatr Soc 1999; 47: 1202-1207.

17. Neyens JCL, Schols JMGA, Dijcks BPJ, Haastregt JCM van, Heuvel WJA van den, Crebolder HFJM, Witte LP de. Development and implementation of a multifactorial intervention for the prevention of falls and fall related injuries in psychogeriatric nursing home patients, a randomised controlled trial. TVV 2002; 26: 24-28.

18. Bueno-Cavanillas A, Padilla-Ruiz F, Jiménez-Moleón JJ, Peinado-Alonso CA, GálvezVargas R. Risk factors in falls among the elderly according to extrinsic and intrinsic precipitating causes. Eur J Epidemiol 2000; 16: 849-859.

19. Krueger PD, Brazil K, Lohfeld LH. Risk factors for falls and injuries in a long-term care facility in Ontario. Can J Public Health 2001; 92: 117-120.

20. Kiely DK, Kiel DP, Burrows AB, Lipsitz LA. Identifying nursing home residents at risk for falling. J Am Geriatr Soc 1998; 46: 551-555.

21. Thapa PB, Gideon P, Brockman KG, Fought RL, Ray WA. Clinical and biomechanical measures of balance as fall predictors in ambulatory nursing home residents. J Gerontol A Biol Sci Med Sci 1996; 51A: M239-M246.

22. Myers AH, Baker SP, Van Natta ML, Abbey H, Robinson EG. Risk factors associated with falls and injuries among elderly institutionalized persons. Am J Epidemiol 1991; 133: 1179-1190.

23. Lipsitz LA, Jonsson PV, Kelley MM, Koestner JS. Causes and correlates of recurrent falls in ambulatory frail elderly. J Gerontol: 1991; 46: M114-M122.

24. Luukinen H, Koski K, Laippala P, Kivela SL. Risk factors for recurrent falls in the elderly in long-term institutional care. Pub Health 1995; 109: 57-65.

25. Maki BE. Gait changes in older adults: predictors of alls or indicators of fear? I Am Geriatr Soc 1997; 45: 313-320.

26. Ooi WL, Hossain M, Lipsitz LA. The assocation between orthostatic hypotension and recurrent falls in nursing home residents. Am J Med 2000; 108: 106-111.

27. Yip YB, Cumming RG. The association between medications and falls in Australian nursing-home residents. Med J Aust 1994; 160: 14-18. 



\section{Chapter 8}

\section{General discussion}


Chapter 8 


\section{INTRODUCTION}

Falls and fall-related injuries are a major problem in people over 65 years of age, especially demented elderly residing in long-term care. Despite the magnitude of the problem of falls in psychogeriatric nursing home residents, only limited evidence is available for the effectiveness and feasibility of fall prevention activities among these residents. ${ }^{1,2}$ Additional research into this topic is needed because the negative consequences of falls for the health status, autonomy and quality of live of these residents must not be underestimated. The implementation of effective fall prevention policy ties in with the current trend to improve the quality of care in Dutch nursing homes and will contribute to the wellbeing of residents.

In this thesis we report on a new multidisciplinary, multifactorial intervention to prevent falls and related injuries in psychogeriatric nursing home patients in the Netherlands. The intervention is based on scientific evidence from the literature and the expertise of Dutch researchers and health care professionals in the fields of falling, fall prevention, guideline development and implementation. The intervention programme consists of a general medical assessment focusing on fall risks and an additional, specific fall risk evaluation tool, applied by a multidisciplinary fall prevention team, both resulting in general and individually tailored fall prevention activities. In a randomised controlled clinical trial of the intervention, described elsewhere in this thesis, we assessed its effectiveness and feasibility in daily nursing home practice. This thesis also aims to contribute to the development of evidence-based guidelines on fall prevention for residents in long-term care facilities.

In this thesis, we presented four interrelated studies:

- a survey of the magnitude of the problem of falls in Dutch nursing homes;

- a systematic review of the evidence for the effectiveness and feasibility of interventions to prevent falls in long-term care facilities;

- an evaluation of the effectiveness of a new multifactorial intervention to prevent falls and fall-related injuries in psychogeriatric nursing home patients (randomised controlled trial);

- a process evaluation to assess the feasibility of this new multifactorial fall prevention programme in daily nursing home care.

In addition, the thesis described the development of the fall risk evaluation tool for demented patients, which is a component of the multidisciplinary intervention programme designed by us. Finally, we looked at the impact of a new national guideline on fall prevention in elderly nursing home residents. 
In this general discussion, we begin with an overview of the main findings of the four studies mentioned above. We then discuss various issues regarding these studies. After summarising the main conclusions of the thesis, we give recommendations for health care practice, fall prevention policy and future research.

\section{MAIN FINDINGS}

\section{Survey of the magnitude of the problem of falls in Dutch nursing homes}

The survey shows that falls are frequent in Dutch nursing homes: almost 2 falls per bed per year, of which $1.3 \%$ result in a fracture. That is, on average, 23 fractures per 1000 beds per year and 4 fractures per nursing home. ${ }^{3}$ Psychogeriatric patients fall more often than somatic patients. On psychogeriatric wards, the number of falls is about twice as high as the number of falls on somatic wards. The majority of fall incidents occur in the afternoon and early evening, and most falls occur in the bedroom. ${ }^{4}$ Intrinsic risk factors for falling appear to be the most important causes of falls. ${ }^{4}$ These findings on the situation in Dutch nursing homes agree with international data on falls in long-term care facilities, that psychogeriatric patients have the highest risk of falling. The survey also reveals that, at present, very few nursing homes in the Netherlands actually use a fall prevention protocol. ${ }^{4}$

\section{Systematic review}

We conducted a systematic review of the literature to assess the effectiveness and feasibility of interventions to prevent falls in elderly people in long-term care facilities. $^{2}$ Eight of the 13 trials included in the survey are multifactorial, multidisciplinary and individually tailored. Only four of which three are multifactorial, report significant favourable effects on falls and/or injurious falls. These are: a comprehensive structured individual assessment with specific safety recommendations; a multidisciplinary programme including both general and resident-specific tailored strategies; a multifaceted nonpharmaceutical intervention; and calcium plus vitamin D supplementation. ${ }^{5-11}$ Remarkably, evaluations of two almost identical programmes, by Ray et al. in 1997 and 2005, give contrary results while another trial, by Kerse et al. in 2004, using a fall risk assessment tool, high risk logo and strategies to address identified risks, even has adverse effects. ${ }^{5,12,13}$

We conclude that, at present, there is still a lack of consistent evidence on the effectiveness of fall prevention interventions in long-term care facilities, 
information regarding the feasibility of these interventions is in general scarce, and although demented elderly are most at risk, the diagnosis of dementia is often an exclusion criterion for participation in research. Due to the limited and inconsistent evidence available, we could not formulate a definite conclusion about which types of programmes effectively reduce falls and related injuries among residents in long-term care facilities. We recommend that health care professionals who wish to reduce falls among persons in long-term care facilities develop tailor-made interventions for their specific setting and population. These interventions should preferably be based on the programmes that have shown significant positive effects.

\section{Effect evaluation: randomised controlled trial}

We conducted a randomised controlled trial of the new multifactorial intervention, described elsewhere in this thesis, to evaluate its effectiveness on the prevention of falls and fall-related injuries in psychogeriatric nursing home patients in the Netherlands. ${ }^{14}$ During twelve months of follow-up, the trial showed that there were 355 falls in 169.5 patient years in the 6 intervention wards and 422 falls in 166.3 patient years in the 6 control wards. The number of injurious falls was 0.69 per patient per year in the intervention wards and 0.67 in the control wards. Intention-to-treat analysis showed that the intervention wards had a significantly lower mean fall incidence rate than the control wards. Subgroup analyses revealed that fall risk declined further as patients participated for a longer time in the intervention programme. The intention-to-treat analysis also showed a lower mean incidence rate for injurious falls, but this difference did not attain statistical significance.

We conclude that the introduction of a multifactorial intervention to prevent falls in psychogeriatric nursing home patients significantly reduces the number of falls. This reduction is substantial and of high clinical relevance. We could not demonstrate a significant effect on injurious falls, but there is an obvious positive tendency.

\section{Process evaluation}

In order to assess the feasibility of the multifactorial intervention to prevent falls and related injuries a process evaluation of its implementation was performed next to the effect evaluation. ${ }^{15}$ Although the results of this study showed that the intervention was not performed entirely according to protocol, it was effective. Protocol deviations were mainly observed with regard to the assessment of environmental risk and the recording of recommendations resulting from the patient assessments. Lack of time, extra workload and poor communication 
between nursing staff and fall prevention team did indeed hamper the implementation process. Factors, which may have positively influenced the implementation process, were: the good communication and close cooperation between the different disciplines of the fall prevention team, their high motivation, the fall prevention meetings and the growing awareness of risk factors for falling.

We conclude that this process evaluation proves that implementing a fall prevention intervention targeted at psychogeriatric patients in a nursing home setting is possible, but certainly not easy, especially in tightly staffed healthcare facilities. Based on the favourable effects on falls and since the randomised controlled trial has shown that the intervention is more effective when implemented during a longer time, and keeping in mind the results of the related process evaluation, we recommend to further improve the implementation of the programme with regard to daily nursing home practice by: simplifying the recording system, the establishment of a fall prevention team, paying more attention to communication between the professionals who are directly involved in the intervention programme and those who are more indirectly confronted with the programme, adding a more detailed introduction programme to the protocol, and a temporarily increase in staff. This intervention is also likely to be suitable for use in other settings like low-care residential homes for older people and geriatric wards in hospitals, but may need to be adapted to specific circumstances.

\section{Methodological and theoretical issues}

\section{Survey of the magnitude of the problem of falls in Dutch nursing homes}

The survey into the incidence of falls and related injuries in Dutch nursing homes gives an up-to-date, representative overview of the reported incidence of falls and fall-related fractures and the use of fall protocols. ${ }^{3,4}$ Previous studies into the Dutch situation are outdated. The survey indicates that psychogeriatric nursing home patients now seem to have a lower fall incidence rate than two decades ago. ${ }^{16,17}$ This reduction can be explained in part by differences in the study population and the fact that the previous studies were not representative for Dutch nursing homes. Another explanation for this difference might be that interest in fall prevention in these settings has increased. The survey however has some limitations. We must emphasize that this survey reveals only the number of reported falls and fall-related fractures. Although nursing homes are obliged to register all fall incidents, underreporting is a real possibility and the actual number of fall incidents and fractures might be higher. In the survey, 
fewer somatic nursing homes were included than nationwide numbers would lead to expect, while combined and psychogeriatric nursing homes were slightly overrepresented. Somatic patients have a lower risk for falling than psychogeriatric patients. This could mean that the total number of fall incidents per nursing home in the Netherlands might actually be slightly lower than the numbers reported in the questionnaire. On the other hand, it is possible that the responding nursing homes were more interested in fall prevention and that they were carrying out more fall prevention activities than non-responding homes. In that case, we might have found higher incidence numbers if the data of all nursing homes had been available. Only three responding nursing homes reported having a fall prevention protocol. ${ }^{4}$ Although the lack of fall prevention protocols does not necessarily mean that no fall prevention activities are carried out, it does indicate that many nursing homes still have an inadequate policy on fall prevention. All in all, then the chance that we misinterpreted the results, due to the possibilities mentioned above, was probably small. Moreover, a strong point of the survey was the relatively high response rate of $54 \%$. This is above average for the applied research method and was enough to produce reliable conclusions. ${ }^{18}$

\section{Systematic review}

It was clear from the systematic review, in which we looked at the outcomes of fall prevention programmes, that evaluation studies are scarce and that effectiveness is questioned. Most studies were underpowered to detect significant effects on injurious falls; apparently, power calculations were based on the expected effect on falls. In six of the eleven trials, which included an assessment of the effect on injurious falls, the control group scored better than the intervention group. Although the differences were small, it is unlikely that a higher power would have revealed more significantly favourable effects on injurious falls. The review shows how important a process evaluation might be to understand the (lack of) effectiveness of a new intervention because an effective fall prevention programme can even have adverse effects when it turns out to be infeasible for the setting in which it is implemented. ${ }^{13}$

The systematic review had some limitations. First, we did not specifically assess the methodological quality of the trials included. Considering the limited number of studies available, however, and the fact that we included only randomised controlled trials, a quality assessment would have produced little additional value. Second, we did not perform a meta-analysis as we felt pooling data would be inappropriate in view of the heterogeneity of the programmes, settings and participating populations. In theory, we may therefore have 


\section{Chapter 8}

underestimated the effects of the programmes on falls resulting in injury. Third, it is possible that we overlooked relevant studies, in particular publications about the programmes' feasibility. This type of information is not always published in official scientific journals and might therefore be difficult for international readers to access.

\section{Effect evaluation}

Regarding the randomised controlled trial we conducted, there are some methodological points that need consideration. ${ }^{14}$ First, the intervention and control groups differed considerably on the variable "number of injurious falls in the twelve months preceding the inclusion period". As a result adjustment for this variable influenced the estimated effect of the intervention on injurious falls. The difference between the two groups may indicate that the intervention group consisted of patients with a higher risk for injurious falls. It is also possible that these differences in injurious falls at baseline were chance fluctuations, thus leading to an overestimation of the effects on injurious falls. A second point that needs to be considered is the possibility that we unintentionally selected the more motivated nursing homes for participation in our trial, as nursing homes that are not interested in fall prevention might not have agreed to participate. For this reason, it remains uncertain whether the programme would be effective in homes where staff might be less motivated to tackle this problem. Thirdly, the data collection in this study was not blinded. Therefore, the real number of fall incidents may have been higher due to underreporting. With regard to the enthusiasm of the members of the fall prevention teams, each willing to deal with the problem of falling in the interest of the residents, it is our opinion that the numbers are reliable. Finally, the power calculation was based on detecting a 30\% difference in falls, which made this trial underpowered for detecting a significant difference in injurious falls.

\section{Process evaluation}

Protocols are often prone not to be used. Therefore, we invested considerable time and energy into designing a multifactorial intervention programme because we are convinced that a successful intervention strongly depends on the ease of implementation by nursing homes themselves, using their own means and regular nursing home staff. ${ }^{14,19,20}$ Although the intervention programme was developed in close cooperation with professionals in the field and was pilot tested on two psychogeriatric wards, the execution of the programme nevertheless raised problems for the nursing homes involved.

Previous studies show that multifactorial fall prevention interventions can

reduce the number of falls in long-term care institutions. ${ }^{1}$ These interventions 
rely heavily on external funding and staff. ${ }^{12}$ The intervention in this study relies on the nursing homes' own means and own professional staff instead of on an academically based team from outside the nursing homes. ${ }^{14,20}$ We believe this is a positive approach as it proves that nursing home personnel is interested and motivated to execute such an intervention.

At the same time we have to realise that any change in daily routines is always difficult for the professionals involved. Even though the nursing home management did not report understaffing, ward staff reported feeling that the intervention raises the pressure of their workload. Likewise, we did not anticipate the difficulties encountered with the recording forms used during the trial, which appeared to be a source for 'non-compliance'. Abundant recording for research purposes may hamper the execution of a meaningful intervention. Recording is not a core responsibility of healthcare professionals and will therefore receive less priority. In the pilot study, these recording forms should have been tested better and their consequences for existing recording systems analysed more thoroughly.

Combining fall prevention meetings with the regular existing multidisciplinary meetings was found to be difficult in the daily routine. But holding separate meetings can be a major impediment to implementation, as it often gives rise to miscommunication between the fall prevention team and the rest of ward staff and decreases compliance with the intervention protocol. As a result, the programme and in particular the advice of the fall prevention teams are not carried out as well as they could have been. In other words, the positive outcome of our study might have been even better with improved implementation.

Nursing staff response to the written questionnaire was only $30 \%$. This is too low for our purposes. The response might have been higher if we had sent the questionnaires to each nurse personally instead of leaving a stack of them on the participating wards. In addition, we did not send out reminders. The outcomes might not therefore be representative for the total group of ward nurses and the questionnaire results should be interpreted with care.

To enhance successful implementation researchers contacted the fall prevention teams twice a month visiting them on location, or phoning or sending e-mails. The high contact level was much appreciated by team members and may have had a positive effect on executing the intervention. When the programme is implemented in regular care, however, this level of support will not be available. It is possible that the number of protocol deviations would have been higher with lower levels of support from the researchers.

\section{Conclusions and recommendations}

Due to the relatively high risk of falls, fall-related morbidity and fall-related 


\section{Chapter 8}

mortality in psychogeriatric patients, it is important to pay extra attention to fall prevention in this group of patients.

The results of this trial provide new evidence for the effectiveness of multifactorial fall prevention programmes in nursing homes. ${ }^{14}$ We conclude that the number of falls in psychogeriatric nursing home residents in the Netherlands can be significantly reduced by a multifactorial fall prevention intervention consisting of a general medical assessment and a fall risk evaluation tool, both resulting in related fall prevention activities. ${ }^{14,20}$ We also conclude that this fall prevention programme is feasible for daily nursing home practice. ${ }^{15}$ Indeed, the outcomes of the study could have been even better with improved implementation.

The results of the studies presented in this thesis have contributed to the development of evidence-based guidelines regarding the prevention of falls among residents in long-term care facilities in the Netherlands. ${ }^{21}$ We were involved in drawing up the first national guideline on fall prevention in the elderly, of which we summarised the essentials for nursing homes in chapter 7 of this thesis. ${ }^{22}$

At present, Dutch nursing homes lack some of the essential preconditions for clinical research on location. Multidisciplinary data collection particularly causes problems. It takes a lot of extra time, energy and effort to obtain complete data. This is offset to a large degree by the enthusiasm of the professionals involved, which surely contributed to completion of the trial. Moreover, the intervention study raised awareness of the "fall problem" and of the need to do something about it.

In the Netherlands, very little research is done in nursing home settings. The fact that we carried out and completed a randomised controlled trial on fall prevention in nursing homes counts as quite a feat; the fact that we targeted psychogeriatric patients makes our research even more remarkable.

\section{Recommendations for long-term care practice}

The results of this randomised controlled trial prove that a fall prevention programme targeted at psychogeriatric patients in a nursing home setting can be possible and effective. Although it is not an easy task to execute a fall prevention trial in an institutional setting, we are convinced that implementing this intervention may contribute to a better quality of care in nursing homes and, importantly, to improved wellbeing of the demented residents. The high rate of falls in nursing homes should no longer be regarded as inevitable.

Nevertheless, various aspects of execution and implementation need to be improved further if it is to tie in seamlessly with daily nursing home practice. This leads us to make a number of recommendations.

Special attention must be given to adequate planning of sufficient manpower, 
good communication and active monitoring of correct implementation.

Well-functioning fall prevention teams contributed to the success of the intervention. We therefore recommend that such a team is set up to guide at least at the start the implementation process. It is important that this is combined with extra effort to involve the whole ward care team.

The recording of the results of the assessments and the fall prevention activities should be integrated into existing recordkeeping to reduce the administrative burden for care professionals. This may require some adaptations to existing forms.

Health care professionals who aim to reduce falls in long-term care facilities are advised to use tailor-made prevention interventions for their specific setting and population. We also advise that such interventions are based on programmes that have shown favourable effects. We strongly recommend that such prevention programmes are first implemented on a small scale, in order to assess its feasibility in that particular setting and to improve weak elements in the programme.

\section{Recommendations for fall prevention policy in Dutch nursing homes}

Although the lack of fall prevention protocols does not necessarily mean that no fall prevention activities are undertaken, it does suggest that nursing homes in the Netherlands have an inadequate policy on fall prevention. ${ }^{4}$ Therefore, we recommend implementing the intervention programme tested in this study, of which essential parts have already been incorporated in the national guideline "Prevention of fall incidents in the elderly". ${ }^{21,22}$ This guideline can help direct nursing homes' attention to the prevention of falls and fall-related injuries in a qualitatively well-founded way. Now, and in addition to the guideline, there is sufficient evidence to recommend vitamin $\mathrm{D}$, preferably combined with $\mathrm{Ca}$, both in high doses, to decrease the fracture incidence in high risk populations like nursing home residents. ${ }^{23,24}$

An essential element of fall prevention policy is an adequate recording of fall incidents and near-accidents in long-term care facilities. Registration must be followed routinely by an analysis to adjust or correct the fall prevention policy. The Dutch Health Care Inspectorate (IGZ) now requires health care facilities to pay more attention to fall prevention policy and the fall incidence rate will become a quality of care indicator in the future. At present, the government stimulates and supports health care providers to implement fall prevention programmes based on scientific evidence. The intervention programme in this thesis is incorporated in the national programme on improving long-term care "Zorg voor Beter" ("Care for Better"). ${ }^{25}$ We recommend, that records on 


\section{Chapter 8}

falls are analysed and reported annually. Such report has to be accessible for the general public.

\section{Recommendations for future research}

Ultimately, we have three recommendations for future research. Firstly, forthcoming research should investigate the effectiveness and feasibility of the intervention tested in this study in other settings like low-care residential homes for older people, geriatric wards in hospitals and institutional settings for people with a mental disability.

Our systematic review has shown that only limited information is available about the feasibility of newly developed interventions. A process evaluation is an important element, although often neglected, in evaluation research. ${ }^{26}$ Secondly, we therefore recommend to combine effect studies in the domain of fall prevention more structurally with process evaluations; especially since differences in implementation of an (effective) intervention can lead to contrary effects., ${ }^{5,12}$

Finally, we recommend that future research should also seek to establish whether fall prevention has favourable effects on fall-related injuries and should include an evaluation of economic costs and benefits. 


\section{References}

1. Gillespie LD, Gillespie WJ, Robertson MC, Lamb SE, Cumming RG, Rowe BH. Intervention for preventing falls in elderly people. The Cochrane Library, Issue2, 2004.

2. Neyens JCL, Haastregt JCM van, Dijcks BPJ, Heuvel WJA van den, Witte LP de, Schols JMGA. Effectiveness and feasibility of interventions for preventing falls in elderly people in long-term care facilities: a systematic review. Submitted

3. Dijcks BPJ, Neyens JCL, Schols JMGA, van Haastregt JCM, Crebolder HFJM, de Witte LP. Valincidenten in verpleeghuizen: gemiddeld bijna 2 per bed per jaar met bij 1,3\% een fractuur als gevolg. Ned Tijdschr Geneeskd 2005;149:1043-7.

4. Dijcks BPJ, Neyens JCL. Valincidenten in verpleeghuizen 2000-2001. Hoensbroek: iRv; 2002.

5. Ray WA, Taylor JA, Weador KG et al. A randomized trial of a consultation service to reduce falls in nursing homes. JAMA 1997;278:557-562.

6. Mc Murdo MET, Millar AM, Daly F. A randomized controlled trial of fall prevention strategies in old peoples' homes. Gerontology 2000;46:83-87.

7. Jensen J, Lundin-Olsson L, Nyberg L et al. Fall and injury prevention in older people living in residential care facilities. Ann Intern Med 2002;136:733-741.

8. Jensen J, Nyberg L, Gustafson $Y$ et al. Fall and injury prevention in residential care. Effects in residents with higher and lower levels of cognition. J Am Geriatr Soc 2003;5:627635.

9. Jensen J, Nyberg L, Rosendahl E et al. Effects of a fall prevention program including exercise on mobility and falls in frail older people living in residential care facilities. Aging Clin Exp Res 2004;16:283-292.

10. Becker $\mathrm{C}$, Kron M, Lindemann $U$ et al. Effectiveness of a multifaceted intervention on falls in nursing home residents. J Am Geriatr Soc 2003;51:306-313.

11. Bischoff HA, Stahelin HB, Dick W et al. Effects of vitamin D and calcium supplementation on falls: a RCT. J Bone Miner Res 2003;18(2):343-351.

12. Ray WA, Taylor JA, Brown AK et al. Prevention of fall-related injuries in long-term care. A randomized controlled trial of staff education. Arch Intern Med. 2005;165:2293-98.

13. Kerse N, Butler M, Robinson E et al. Fall prevention in residential care: a cluster, randomised, controlled trial. J Am Geriatr Soc 2004;52:524-531.

14. Neyens JCL, Dijcks BPJ, Twisk JW, , Schols JMGA, Haastregt JCM van, Heuvel WJA van den, Witte LP de. A multifactorial intervention for the prevention of falls and related injuries in psychogeriatric nursing home patients, a randomised controlled trial (RCT). Submitted.

15. Neyens JCL, Bleijlevens MHC, Dijcks BPJ, Haastregt JCM van, Witte LP de, Schols JMGA, Heuvel WJA van den. Feasibility of a multifactorial fall prevention programme for psychogeriatric nursing home patients. Submitted. 


\section{Chapter 8}

16. Vermeulen HBM (1994). Vallen en valpreventie in het verpleeghuis: een interventiestudie naar het effect van een bed-alarm-systeem. Nijmegen, PhD thesis Katholieke Universiteit Nijmegen.

17. Dijk van PTM, Meulenburg OGRM, Sande van de HJ et al. Falls in dementia patients. Gerontologist 1993;33(2):200-204.

18. Eaker S, Bergstróm R, Bergström A, Adami H-O, Nyren O. Response Rate to mailed epidemiologic questionnaires: a population-based randomized trial of variations in design and mailing routines. Am J Epidemiol 1998; Vol. 147, No.1.

19. Neyens JCL, Dijcks BPJ, Schols JMGA, Haastregt JCM van, Heuvel WJA van den, Crebolder HFJM, Witte LP de. Ontwikkeling en implementatie van een multifactoriële interventie gericht op de preventie van valincidenten en de gevolgen daarvan bij psychogeriatrische verpleeghuispatiënten. TVV 2002;26:24-28.

20. Neyens JCL, Dijcks BPJ, van Haastregt JCM, Witte LP de, van de Heuvel WJA, Crebolder HFJM, Schols JMGA. The development of a multidisciplinary fall risk evaluation tool for demented nursing home patients in the Netherlands. BMC Public Health 2006;6:74.

21. $\mathrm{CBO}$, Kwaliteitsinstituut voor de Gezondheidszorg. Richtlijn Preventie van valincidenten bij ouderen. Alphen aan de Rijn: Van Zuiden Communications BV, 2004.

22. Neyens JCL, Dijcks BPJ, Kinkelder A de, Graafmans WC, Schols JMGA. CBO richtlijn: Preventie van valincidenten bij ouderen; wat kunnen verpleeghuizen hiermee? TCG 2005;36:155-160.

23. Bischoff-Ferrari HA, Willett WC, Wong JB, Giovannucci E et al. Fracture prevention with vitamin D supplementation: a meta-analysis of randomized controlled trials. JAMA 2005;293:2257-2264.

24. Boonen S, Lips P, Bouillon R et al. Need for additional calcium to reduce the risk of hip fractures with vitamin $d$ supplementation: evidence from a comparative meta-analysis of randomized controlled trials. J Clin Endocrinol Metab 2007 Apr;92(4):1415-23.

25. www.zorgvoorbeter.nl

26. Oakley A, Strange V, Bonell C, Allen E, Stephenson J, RIPPLE Study Team. Process evaluation in randomised controlled trials of complex interventions. BMJ 2006; 332: 413-6. 

Summary 
Falls occur in all age groups, but they are a particularly large health problem in the elderly. A fall is defined as an event which causes some one to unintentionally come into contact with the ground or some lower level, regardless of the cause. One-third of people aged 65 and older and living independently have at least one fall a year. Fall incidents are even more frequent in homes for the elderly and nursing homes: half of all residents fall at least once a year. Residents with dementia are at particular risk of falling and this group has the highest fall incidence.

The consequences of a fall may be physical, psychological or social. About $10 \%$ of falls in the elderly result in serious injury. Those in residential care, including nursing homes, have a much greater chance of sustaining a hip fracture after a fall than independently living elderly. It has been found that mortality after a hip fracture is relatively high. Common psychological consequences are: fear of falling, afraid to move around (loss of self-confidence), depression and feelings of helplessness. Social consequences, commonly lower participation in social activities and social isolation, are primarily associated with reduced mobility after a fall. Clearly then, falls increase the frailty and vulnerability of nursing home patients. It is also important to consider the high healthcare costs of falls. Finally, falls, especially those resulting in hip fracture, also increase a patient's need for care and, by extension, nursing staff's workload.

Fall incidents and their consequences thus have a considerable negative impact on patients' autonomy and quality of life. They cause further decline in patients' functional limitations and increase morbidity, mortality and medical consumption. For these reasons, it is vital that preventive measures are taken against fall incidents in nursing home populations. Research has shown that preventive interventions can help reduce both the number and severity of falls. A multifactorial approach is required to tackle the many different factors that influence fall risk in nursing homes. Such a programme should be carried out by multidisciplinary teams, which are present in all Dutch nursing homes.

The four main objectives of this doctorate research were:

- to raise awareness among nursing home staff about the scale of the fall problem in nursing home populations, and especially in psychogeriatric patients;

- to summarise findings in the literature about the effectiveness and feasibility of fall prevention interventions in nursing homes;

- to evaluate the effectiveness and feasibility of a new fall prevention 
intervention for psychogeriatric nursing home patients;

- to contribute to the development of national guidelines for preventing falls in the elderly, with special attention to fall prevention in nursing home patients.

The main research questions addressed in this dissertation are:

- how effective is the new, multifactorial fall prevention intervention in psychogeriatric nursing home patients?

- is this intervention feasible for inclusion in routine nursing home care?

Our research objectives and questions were explored and described in four separate studies:

- a mail survey of all Dutch nursing homes to identify the size of the fall problem in Dutch nursing homes;

- a systematic review of the literature on the effectiveness and feasibility of fall prevention interventions in nursing homes;

- an effect evaluation of a new multifactorial intervention developed by us to prevent falls in nursing homes;

- a process evaluation of the same intervention.

Chapter 1 comprises an introduction to the research subject. We describe general fall incidence rates and specific data for the Netherlands, risk factors for falling, fall prevention guidelines and the characteristics of nursing homes in the Netherlands. We also present the main research objectives and research questions.

In chapter 2, we report on the mail survey of all Dutch nursing homes to determine the annual fall incidence and the number of fall-related fractures. As a result of the survey, we found that the fall incidence in nursing homes was almost 2 falls per bed per year. Fall incidence per bed was highest for psychogeriatric patients. In each nursing home, an average of four falls per year resulted in fractures. The annual number of fractures caused by falls, on average, was 23 per 1000 beds. About $1.3 \%$ of falls resulted in fracture, with no clear differences between somatic and psychogeriatric patients.

In chapter 3, we present the results of our systematic review of the literature on the effects and practical feasibility of fall prevention interventions in nursing homes. Thirteen studies (randomised controlled trials) were included in the review. Most studies were multifactorial $(n=10)$ and multidisciplinary $(n=8)$. Fall evaluations were used in 8 of the 13 studies, and individualised 
prevention programmes, tailored to the patient's situation and condition, in nine studies. Significant positive effects were reported in only four studies, of which three were multifactorial. These four studies were: an individual multifactorial fall-risk evaluation combined with specific safety recommendations; a multidisciplinary programme containing both general and specific prevention measures; a multifactorial non-pharmaceutical intervention; and calcium plus vitamin D supplementation. An important conclusion of our systematic review was that, in many cases, the evidence was not strong and sometimes even inconsistent and that few publications gave process information about the implementation of the programmes.

In chapter 4 we describe the development of a specific fall risk evaluation for psychogeriatric patients, which is part of a new multifactorial fall prevention intervention. The evaluation's design was based on the most recent data from the literature and also tied in as much as possible with existing medical and paramedical routines in Dutch nursing homes. In the preparatory phase, we consulted a group of Dutch experts on falling, fall prevention and implementation processes. The fall risk evaluation was made up of the following components: fall history, medication used, locomotor functioning, correct use of aids and correct use of protective devices. The evaluation was implemented by multidisciplinary teams, which are present in all Dutch nursing homes.

In chapter 5, we present the results of the effect evaluation of the new multifactorial fall prevention intervention. The study was a randomised controlled trial with a follow-up after 12 months. It was carried out in 12 nursing homes, with one psychogeriatric ward from each nursing home actually participating in the study. Outcome measures were the number of fall incidents and the number of fall-related injuries. The study had 518 participants (249 patients in the intervention group and 269 in the control group). In the intervention group, 355 falls were recorded over 169.5 patient years (equivalent to 2.09 falls per patient per year). The intention-to-treat analysis, corrected for patient characteristics, ward characteristics and intracluster correlation, showed that the intervention group had a significantly lower average fall incidence than the control group (rate ratio $=0.64,95 \% \mathrm{Cl}=0.43-0.96, \mathrm{p}=0.029$ ). Subgroup analyses showed that fall risk reduced further the longer patients participated in the study. The number of fallrelated injuries was 0.69 per patient per year in the intervention group and 0.67 in the control group. Intention-to-treat analysis revealed a lower mean for fallrelated injuries in the intervention group, but this difference was not statistically significant (rate ratio $0.54,95 \mathrm{Bl}=0.29-1.01, \mathrm{p}=0.054$ ). 
In chapter 6, we present the results of the process evaluation carried out with the effect study. We found that the intervention was largely carried out according to protocol. The multidisciplinary fall prevention teams established specifically for the purposes of our trial functioned well and were highly motivated. Communication between these teams and the rest of the ward staff was, however, not as good as it should have been. Also, the extra registration forms that needed to be filled in for the study were not well-incorporated in daily routines on the ward. The main barriers to optimum implementation were lack of time to implement the intervention in full and inadequate communication between the fall prevention teams and the rest of the ward staff. Nevertheless, the intervention was effective. We concluded that the intervention is feasible for implementation in nursing homes.

During the research period covered by this dissertation, we also collaborated on the drawing up of the first national CBO guidelines 'Prevention of fall incidents in the elderly' which were published in November 2004. The essential components of our intervention were included in the guidelines before the effect study had been completed. In chapter 7, we go into the importance of these guidelines for nursing homes. Specifically, we address the problem of falls in nursing homes and the associated findings and recommendations of the guidelines.

Chapter 8, finally, contains a summary of the most important findings of the four different studies conducted: (1) a mail survey of all Dutch nursing homes to identify the problem of falls in nursing homes, (2) a systematic review of the literature on the effectiveness and applicability of fall prevention interventions in nursing homes, (3) an effect evaluation of a new multifactorial fall prevention intervention developed by us, and (4) a process evaluation of that intervention. The results of these studies are discussed and specific methodological issues addressed. We then make recommendations for institutions for chronic care, for fall prevention policy in nursing homes and for future research.

The main conclusions are:

- we found new evidence of the effectiveness of a multifactorial intervention aimed at preventing falls in psychogeriatric nursing home patients;

- the new intervention is feasible for implementation in nursing homes, although improvements to the implementation process are desirable.

The multifactorial intervention described in this dissertation has been included in the national programme on improving long-term care (Zorg voor Beter), with the aim of realising fall prevention activities on a wide scale in nursing homes and other institutions for chronic care. 

Samenvatting 
Vallen komt in alle leeftijdsgroepen voor, maar vormt vooral bij ouderen een groot gezondheidsprobleem. Onder een val wordt verstaan een gebeurtenis waarbij de betrokkene onbedoeld op de grond of een lager niveau terechtkomt, ongeacht de oorzaak. Een derde van de zelfstandig wonende ouderen van 65 jaar en ouder valt één keer of vaker per jaar. Ook in zorginstellingen komen valincidenten veelvuldig voor. In verzorgings- en verpleeghuizen valt de helft van de bewoners tenminste één keer per jaar. Vooral dementerende cliënten lopen een relatief groot risico om te vallen. In deze groep is de valincidentie het hoogst.

De gevolgen van vallen kunnen worden ingedeeld in lichamelijke, psychische en sociale gevolgen. Ongeveer tien procent van de valpartijen bij ouderen leidt tot ernstige letsels. De kans op een heupfractuur bij bewoners van verpleeg- en verzorgingshuizen is vele malen groter dan bij zelfstandig wonende ouderen. Gebleken is dat na een heupfractuur de kans om hieraan te overlijden relatief groot is. Psychische gevolgen zijn vooral valangst, angst om te bewegen (verlies van zelfvertrouwen), depressiviteit en gevoelens van hulpeloosheid. Sociale gevolgen hangen samen met de verminderde actieradius die vaak leidt tot een lagere deelname aan sociale activiteiten en isolement. Het zal duidelijk zijn dat de al aanwezige fragiliteit van verpleeghuispatiënten door valincidenten alleen maar toeneemt. Ten slotte mogen ook de hoge gezondheidszorgkosten die valincidenten met zich meebrengen niet buiten beschouwing blijven. Daarbij stijgt na een valpartij, zeker als deze resulteert in een heupfractuur, vaak de zorgzwaarte van de patiënt met een secundaire verhoging van de werkdruk van het personeel.

Valincidenten en de gevolgen daarvan hebben dus een aanzienlijk negatieve invloed op de autonomie en kwaliteit van leven van de getroffen patiënten en leiden tot een toename van functionele beperkingen en functionele achteruitgang, morbiditeit, mortaliteit en medische zorgconsumptie. Daarom is het belangrijk preventieve maatregelen te nemen om valincidenten in de verpleeghuispopulatie zoveel mogelijk te voorkomen. Uit onderzoek blijkt dat preventieve interventies bij kunnen dragen aan het verminderen van valincidenten en de negatieve gevolgen daarvan. Omdat tal van factoren van invloed kan zijn op het valrisico in verpleeghuizen, is een multifactoriële aanpak aangewezen. In verpleeghuizen kan deze aanpak multidisciplinair uitgevoerd worden.

Dit proefschrift heeft vier doelstellingen: (1) het verhogen van de aandacht van het personeel voor het valprobleem in verpleeghuizen in het algemeen en bij dementerenden in het bijzonder, (2) het presenteren van een overzicht van de literatuur over de effectiviteit en de bruikbaarheid van valpreventieve interventies in verpleeghuizen, (3) het evalueren van een valpreventieve interventie voor psychogeriatrische verpleeghuispatiënten op effectiviteit en uitvoerbaarheid en 
(4) het bijdragen aan de ontwikkeling van een nationale richtlijn voor valpreventie bij ouderen in het algemeen en bij verpleeghuispatiënten in het bijzonder.

De belangrijkste vraagstellingen van dit proefschrift zijn: (1) hoe effectief is de nieuwe multifactoriële valpreventieve interventie bij psychogeriatrische verpleeghuispatiënten en (2) hoe toepasbaar is deze interventie in de dagelijkse verpleeghuispraktijk?

De doelstellingen en vragen zijn uitgewerkt en beschreven in een viertal studies die we verricht hebben: (1) een schriftelijke enquête onder alle Nederlandse verpleeghuizen om het valprobleem in verpleeghuizen in kaart te brengen, (2) een literatuurstudie (systematic review) betreffende de effectiviteit en de toepasbaarheid van valpreventieve interventies in verpleeghuizen(3) een effect evaluatie van de door ons ontwikkelde nieuwe multifactoriële valpreventieve interventie en (4) een procesevaluatie van diezelfde interventie.

Hoofdstuk 1 betreft de inleiding op het onderwerp en beschrijft de volgende zaken: het voorkomen van valincidenten in het algemeen en in Nederland in het bijzonder, risico factoren voor vallen, richtlijnen valpreventie, en verpleeghuizen in Nederland. Daarnaast worden de belangrijkste vraagstellingen en de doelstellingen van de studie beschreven.

Hoofdstuk 2 rapporteert over een schriftelijke enquête die uitgevoerd is onder alle verpleeghuizen in Nederland om vast te stellen hoeveel valincidenten zich jaarlijks voordoen en hoeveel fracturen ontstaan als gevolg van valincidenten. Er wordt geconcludeerd dat het aantal valincidenten per verpleeghuisbed gemiddeld bijna 2 per jaar is. Het aantal valincidenten per bed was onder de groep psychogeriatrische patiënten groter dan onder de groep somatische patiënten. Er waren per verpleeghuis gemiddeld ongeveer 4 fracturen per jaar als gevolg van valincidenten. Het jaarlijkse aantal fracturen door een valincident was gemiddeld ongeveer 23 per 1000 bedden. Gemiddeld had 1,3\% van de valincidenten een fractuur tot gevolg. Er waren hierbij geen duidelijke verschillen tussen somatische en psychogeriatrische patiënten.

In hoofdstuk 3 worden de resultaten gepresenteerd van een literatuurstudie (systematic review) naar de effecten en de toepasbaarheid van valpreventieve interventies in verpleeghuizen. In de review zijn 13 studies (randomised controlled trials) opgenomen. Het merendeel van de studies is multifactorieel $(n=10)$ en multidisciplinair $(n=8)$. In 8 van de 13 studies wordt een valevaluatie gebruikt en in 9 studies blijken de preventie programma's gemaakt te zijn op maat van de patiënt. Slechts in 4 studies worden significant positieve effecten gerapporteerd. 
Drie van deze 4 studies zijn multifactorieel. De 4 studies betreffen: een individuele multifactoriële valrisico-evaluatie gecombineerd met specifieke aanbevelingen voor veiligheid, een multidisciplinair programma met algemene en specifieke preventieve maatregelen, een multifactoriële niet farmaceutische interventie en het toedienen van calcium + vitamine D. Belangrijk is dat in deze systematische review geconcludeerd wordt dat het bewijs vaak nog niet zo sterk is, soms inconsistent en dat er in het algemeen weinig proces informatie over de implementatie programma's beschikbaar is.

Hoofdstuk 4 beschrijft de ontwikkeling van een specifieke valrisicoevaluatie voor psychogeriatrische patiënten, als onderdeel van een nieuw ontwikkelde multifactoriële valpreventieve interventie. De opzet van deze evaluatie is gebaseerd op de meest recente gegevens uit de wetenschappelijke literatuur en sluit ook zoveel mogelijk aan bij bestaande medische en paramedische routines in Nederlandse verpleeghuizen. In het voorbereidingstraject is een groep Nederlandse experts op de gebieden van vallen, valpreventie en implementatieprocessen betrokken. De valrisico-evaluatie is samengesteld uit de volgende elementen: de valgeschiedenis, de gebruikte medicatie, de locomotorische functie, het (juiste) gebruik van hulpmiddelen en het (juiste) gebruik van beschermmiddelen. De uitvoering ervan wordt gedaan door het multidisciplinaire team dat zo kenmerkend is voor de Nederlandse verpleeghuizen.

In hoofdstuk 5 worden de resultaten gepresenteerd van de effectevaluatie van deze nieuw ontwikkelde multifactoriële valpreventieve interventie. Het betreft een randomised controlled trial met een follow-up van 12 maanden in twaalf verpleeghuizen waarbij steeds van elk verpleeghuis één psychogeriatrische afdeling deelnam. Uitkomstmaten waren het aantal valincidenten en het aantal valgerelateerde letsels. Er namen 518 patiënten deel aan de studie (respectievelijk 249 en 269 in de interventiegroep en in de controlegroep). In de interventiegroep werd 355 keer gevallen in 169,5 patiëntenjaren $(=2,09$ keer per patiënt per jaar), in de controlegroep werd 422 keer gevallen in 166,3 patiëntenjaren $(=2,54$ keer per patiënt per jaar). De intention-to-treat analyse, met correctie voor patiëntenkenmerken, afdelingskenmerken en intracluster correlatie, toonde een statistisch significant lager gemiddelde van de valincidentie aan in de interventiegroep (rate ratio $=0.64,95 \% \mathrm{Cl}=0.43-0.96, \mathrm{p}=0.029)$. Subgroepanalyses toonden een verdere daling van het valrisico als de patiënten langer deelnamen aan het onderzoek. Het aantal valgerelateerde letsels was 0,69 per patiënt per jaar in de interventiegroep en 0,67 in de controlegroep. De intention-to-treat analyse toonde een lager 
gemiddelde van de valgerelateerde letsels in de interventiegroep, maar dit verschil was niet statistisch significant (rate ratio 0.54, $95 \mathrm{Bl}=0.29-1.01, \mathrm{p}=0.054$ ).

Hoofdstuk 6 presenteert de resultaten van de aan de effectstudie gerelateerde procesevaluatie. De interventie bleek overwegend volgens het protocol te zijn uitgevoerd. De specifiek samengestelde multidisciplinaire valpreventieteams functioneerden goed en waren zeer gemotiveerd. De communicatie tussen deze teams en de rest van het afdelingspersoneel was echter niet optimaal. Bovendien pasten de extra registratieformulieren van de studie moeilijk in de gangbare routine van alle dag. De belangrijkste belemmerende factoren voor implementatie waren het gebrek aan voldoende tijd om de interventie compleet uit te voeren en de suboptimale communicatie tussen de valpreventieteams en de rest van het afdelingspersoneel. Desalniettemin is de interventie effectief gebleken. Geconcludeerd wordt dat de interventie uitvoerbaar is in verpleeghuizen.

Gedurende het in deze thesis beschreven onderzoeksproject werkten we mee aan de realisatie van de eerste nationale CBO richtlijn "Preventie van valincidenten bij ouderen", die verscheen in november 2004. De essenties van de door ons ontwikkelde interventie kregen reeds een plek in deze richtlijn vooraleer de effectstudie afgerond was. Hoofdstuk 7 beschrijft de betekenis van deze richtlijn voor verpleeghuizen. We focussen specifiek op de valproblematiek in verpleeghuizen en op de daaraan gerelateerde bevindingen en aanbevelingen van de richtlijn.

In hoofdstuk 8 van dit proefschrift wordt tot slot ingegaan op de belangrijkste bevindingen: (1) een schriftelijke enquête onder alle Nederlandse verpleeghuizen om het valprobleem in verpleeghuizen in kaart te brengen, (2) een literatuurstudie (systematic review) betreffende de effectiviteit en de toepasbaarheid van valpreventieve interventies in verpleeghuizen, (3) een effect evaluatie van de door ons ontwikkelde nieuwe multifactoriële valpreventieve interventie en (4) een procesevaluatie van diezelfde interventie. De resultaten worden bediscussieerd waarbij met name methodologische aspecten aan de orde komen. Vervolgens worden aanbevelingen gedaan voor instellingen voor chronische zorg, voor een valpreventief beleid in verpleeghuizen en voor toekomstig onderzoek.

De belangrijkste conclusies zijn:

- er is nieuw bewijs dat een multifactoriële interventie gericht op valpreventie bij psychogeriatrische verpleeghuispatiënten effectief is 
- de nieuwe interventie is in principe uitvoerbaar in verpleeghuizen, maar verbetering van het implementatieproces is wenselijk

Inmiddels is de multifactoriële interventie, zoals beschreven in dit proefschrift, opgenomen in het landelijke verbeterprogramma "Zorg voor Beter" met als doel een brede implementatie van valpreventieve activiteiten te realiseren in verpleeghuizen en andere instellingen voor chronische zorg. 



\section{Dankwoord}


Dankwoord

162 
Aan de totstandkoming van dit proefschrift hebben velen direct of indirect bijgedragen. Ik wil graag een aantal mensen bedanken die ieder op hun manier hierin een rol hebben gespeeld.

Allereerst wil ik de leden van het promotieteam bedanken: Wim van den Heuvel (promotor), Jos Schols (promotor), Luc de Witte (copromotor en projectleider), Jolanda van Haastregt (co-promotor) en Béatrice Dijcks. Een geweldig team dat mij navigeerde door onderzoeksland, met een gezonde mix van ernst en gezelligheid. Jullie enthousiaste houding ten aanzien van het onderzoek heb ik als zeer prettig ervaren.

Wim, jij was degene die met zachte stem en duidelijke opstelling mijn agenda en deadlines bewaakte en daarmee de voortgang van dit promotietraject. Ik wil je bedanken voor je grote kennis van zaken en je deskundige begeleiding. Je voortdurende steun en de prettige werkomgeving die je op het iRv wist te creëren waren fijn en inspirerend.

Jos, jij was de initiator van het proces dat geleid heeft tot de voltooiing van dit proefschrift. Jouw enthousiasme stimuleerde mij enorm. Je bent een prima begeleider, helder, to the point en op zijn tijd een Bourgondiër. Als ik ergens een probleem van maakte, wist jij het probleem moeiteloos te relativeren. Jouw praktijkkennis en rol in het ondersteunen en adviseren zijn erg belangrijk geweest voor het project. Bedankt voor het eindeloze vertrouwen in me en de kansen die je me biedt. Jouw tomeloze energie en zeer goed geheugen voor kleine details zullen me bijblijven.

Luc, ik bewonder de manier waarop jij op een vriendelijke en rustige manier hele zinnige en bruikbare kritiek kan geven. Je vormde voor mij een prima uitlaatklep, als zaken eens niet liepen zoals ik dat wilde. Jij hielp mij waar nodig relativeren zodat het steeds weer meeviel. Ik heb van jou ook geleerd dat mens-zijn primeert boven wetenschapper-zijn.

Jolanda, ik ben dit promotietraject in kunnen gaan omdat jij me bent voorgegaan. Het is prettig om met jou samen te werken, ook in de Breemakkergaard. Ik heb veel van je geleerd. Bedankt voor het nuttige en zorgvuldige commentaar op de artikelen en je methodologische kennis.

Béatrice, mede dankzij jouw logistieke adviezen is de dataverzameling goed verlopen. Jij waakte ervoor dat de data goed ingevoerd werden en jij zorgde ervoor dat er bruikbare bestanden kwamen. Tevens was je methodologische en statistische kennis zeer nuttig voor het onderzoek. Ik ben je dankbaar voor het kritische commentaar dat je gaf bij de artikelen. Mede door onze wandelpauzes in Hoensbroek hield jij er de beweging in. 
Vervolgens wil ik graag de coauteurs van mijn artikelen bedanken: Michel Bleijlevens, Adriaan de Kinkelder, Wilco Graafmans en in het bijzonder Harry Crebolder en Jos Twisk.

Ik dank de directies en medewerkers van de pilothuizen, de controlehuizen en de interventiehuizen. Verder dank ik alle patiënten die hebben deelgenomen aan dit onderzoek. Ik hoop van harte dat het goed met u gaat.

Ook alle leden van de valpreventieteams van de interventiehuizen wil ik bedanken, die naast hun zeer drukke werkzaamheden in de praktijk, bereid zijn geweest om aan dit onderzoek deel te nemen. Het heeft bijgedragen aan dit mooie klinische onderzoek. Aan mijn frequente bezoeken bij jullie heb ik waardevolle herinneringen overgehouden.

De Raad van Toezicht en de Raad van Bestuur van Stichting De Riethorst Stromenland dank ik voor de geboden mogelijkheden om in en vanuit het verpleeghuis wetenschappelijke activiteiten te ontplooien.

Frank Staal dank ik voor zijn betrokkenheid bij het wel en wee van het onderzoek en de nooit aflatende steun.

Alle overige medewerkers van mijn verpleeghuis alsook onze verzorginghuizen, dank ik voor de ruimte om innovatieve en ook wetenschappelijke activiteiten te ontwikkelen. Sjan Schroevers en Izet Sadžak bedank ik voor hun administratieve ondersteuning.

Al mijn collega's op het iRv wil ik danken voor hun interesse in mijn onderzoek en voor de inspirerende werkomgeving die ze weten te creëren.

Mijn nieuwe collega's van Zorg voor Beter (Utrecht) en van de LPZ (Maastricht) wil ik bedanken voor de prettige ontvangst en aangename werksfeer.

Een speciaal woord van dank voor mijn pa en ma die me onvoorwaardelijk gesteund hebben. Mijn vele logementen, soms onaangekondigd, namen ze er met plezier bij. Hun "bed and breakfast" scheelde mij veel reiskilometers én reistijd.

Dan tot slot, maar niet in de laatste plaats, Mady, Maarten en Sanne waar ik echt trots op ben!

Mady, ik ben jou de meeste dank verschuldigd. Jij hebt mij de nodige ruimte gegeven om de werkzaamheden in het kader van dit proefschrift te kunnen verrichten. Tot op de dag van vandaag heb je steeds begrip opgebracht voor de schaarse tijd die ik in deze periode aan het gezin kon geven. 
Toch genieten we samen van de talenten van onze kinderen. De wielercarrière van Maarten bezorgt ons ontspanning en de artistieke ontplooiing van Sanne bezorgt ons voldoening. Sanne, dankzij jou is het proefschrift extra bijzonder geworden. Jij ontwierp de prachtige omslag waarvoor je zelf mooie materialen uitzocht en zelf ook model stond. Ik vind dit geweldig.

Ik wil Maarten en Sanne nog even apart bedanken voor de hand- en spandiensten die ze thuis regelmatig verrichten om hun moeder te helpen, als ik er weer eens niet ben. 

Curriculum Vitae 
Jacques Neyens werd op 29 augustus 1956 geboren te Bree (België). In 1974 behaalde hij zijn Wetenschappen A diploma aan het Sint Michiels College te Bree. In september 1974 startte hij met de opleiding "Kinesitherapie en Motorische Revalidatie" aan de Katholieke Universiteit Leuven. In 1978 behaalde hij zijn diploma Licentiaat in de Kinesitherapie en Motorische Revalidatie.

In september 1978 werd hij aangesteld als hoofd fysiotherapie in Streekverpleeghuis Sint Agnes (inmiddels, na enkele fusies, Stichting De Riethorst Stromenland) te Geertruidenberg (Nederland).

Als assistent onderzoeker was Jacques regelmatig betrokken bij wetenschappelijk onderzoek in zijn verpleeghuis.

In 2002 startte hij parttime zijn in dit proefschrift beschreven promotieonderzoek aan de Universiteit Maastricht (UM), in samenwerking met het Kenniscentrum voor Revalidatie en Handicap (iRv), te Hoensbroek, en verpleeghuis De Riethorst Stromenland te Geertruidenberg.

Sinds 2003 is hij als gastdocent verbonden aan de Hoge School Utrecht, opleiding fysiotherapie in de geriatrie.

Verder was hij coauteur van de landelijke CBO richtlijn "Preventie van valincidenten bij ouderen" (2004).

Sinds september 2006 is hij kernteamlid van het Zorg voor Beter Verbetertraject Valpreventie.

In januari 2007 werd hij aangesteld als postdoc onderzoeker "Vallen en fixatie" bij de onderzoeksgroep die de Landelijke Prevalentiemeting Zorgproblemen uitvoert (Universiteit Maastricht, onderzoeksinstituut CAPHRI).

Jacques is getrouwd met Mady Van de Weyer, die diëtiste is. Hij is vader van 2 kinderen: Maarten en Sanne. 



978-90-8590-021-4 